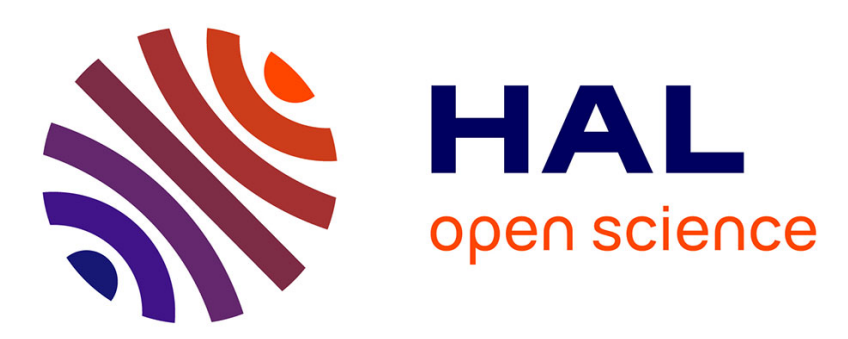

\title{
Scattering of elastic waves in a perturbed isotropic half space with a free boundary. The limiting absorption principle
}

\author{
Y. Dermenjian, J.C. Guillot
}

\section{- To cite this version:}

Y. Dermenjian, J.C. Guillot. Scattering of elastic waves in a perturbed isotropic half space with a free boundary. The limiting absorption principle. RR-0491, INRIA. 1986. inria-00076063

\section{HAL Id: inria-00076063 \\ https://hal.inria.fr/inria-00076063}

Submitted on 24 May 2006

HAL is a multi-disciplinary open access archive for the deposit and dissemination of scientific research documents, whether they are published or not. The documents may come from teaching and research institutions in France or abroad, or from public or private research centers.
L'archive ouverte pluridisciplinaire HAL, est destinée au dépôt et à la diffusion de documents scientifiques de niveau recherche, publiés ou non, émanant des établissements d'enseignement et de recherche français ou étrangers, des laboratoires publics ou privés. 
SCATTERING OF ELASTIC WAVES IN A PERTURBED ISOTROPIC HALF SPACE WITH A FREE BOUNDARY. THE LIMITING ABSORPTION PRINCIPLE.

by

Yves DERMENJIAN and Jean-Claude GUILLOT *

Département de Mathématiques

Centre Scientifique et Polytechnique

Université Paris Nord

93430

Villetaneuse France

* Conseiller scientifique à l'I.N.R.I.A. 
In this article we consider the selfadjoint operator governing the propagation of elastic waves in a perturbed isotropic half space with a free boundary condition. We prove the limiting absorption principle in appropriate Hilbert spaces for this operator. We also prove decreasing properties for the eigenfunctions associated with strictly positive eigenvalues of this operator.

The proofs are based on the limiting absorption principle for the selfadjoint operator governing the propagation of elastic waves in an homogeneous isotropic half space with a free boundary and on the so called division theorem for it. Both perturbations of $\mathbb{R}_{+}^{2}=\left\{\left(x_{1}, x_{2}\right) \in \mathbb{R} ; x_{2}>0\right\}$ and $\mathbb{R}_{+}^{3}=\left\{\left(\mathrm{x}_{1}, \mathrm{x}_{2}, \mathrm{x}_{3}\right) \in \mathbb{R}^{3} ; \mathrm{x}_{3}>0\right\}$ are studied.

Rēsumē

Dans cet article on considère l'opérateur autoadjoint associé à la propagation des ondes élastiques dans un demi espace isotrope perturbé avec la condition de surface libre sur le bord. On démontre le principe d'absorption limite pour cet opérateur. On démontre aussi des propriétés de décroissance des fonctions propres associées aux valeurs propres strictement positives de cet opérateur.

Les démonstrations précédentes reposent sur le principe d'absorption limite pour l'opérateur autoadjoint associé à la propagation des ondes élastiques dans un demi-espace isotrope homogène avec la condition de surface libre sur le bord et sur un théorème de division pour cet opérateur. On considère les deux demi-espaces $\mathbb{R}_{+}^{2}=\left\{\left(x_{1}, x_{2}\right) ; x_{2}>0\right\}$ et $\mathbf{R}_{+}^{3}=\left\{\left(x_{1}, x_{2}, x_{3}\right) ; x_{3}>0\right\}$. 
Key words

Elastic waves - Perturbed isotropic half space with a free boundary The limiting absorption principle.

Mots clés

Ondes élastiques - Demi-espace isotrope perturbé avec la condition de surface libre - Le principe d'absorption limite. 


\section{INTRODUCTION}

This article is the first one of two papers concerned with scattering of elastic waves in a perturbed isotropic half space with a free boundary. More precisely we consider perturbations with compact support of an homogeneous isotropic elastic half space with a free boundary. The perturbations can be bounded obstacles, solid or fluid bounded inclusions, cracks of finite dimensions or an inhomogeneous part of the material.

This problem has its own interest. Usually in non-destructive evaluation of solids one of the main problems is the scattering of elastic waves by a crack of finite dimensions located at the plane (or curved) interface of two isotropic media. In fact the real problem is the inverse scattering one, i.e., the determination of the size, shape and orientation of the crack. Nevertheless the direct scattering problem is a necessary prerequisite for solving the inverse scattering one.

Scattering of elastic waves by cracks has been studied by asymptotic methods in [2]. In this article this problem is studied from the point of view of the mathematical theory of scattering (see [24], [27], [30]). From this point of view the difference between the case of a perturbed isotropic half space with a free boundary and that of two isotropic half spaces with a perturbed plane interface is not very important. The case of a perturbed isotropic half space with a free boundary is simpler. That is the reason why we begin with it here.

In this article we essentially prove the so called limiting absorption principle for the selfadjoint operator governing the propagation of elastic waves in a perturbed isotropic half space with a free boundary. This selfadjoint operator is denoted by $A$. In the case of a homogeneous isotropic half space with a free boundary the selfadjoint operator is denoted by $\mathrm{A}_{0}$. 
The limiting absorption principle is a stationnary method used in the spectral theory of selfadjoint operators associated with the partial differential equations of the mathematical physics.

To prove the limiting absorption principle we used the methods of S. Agmon [3] (see also [18], [23], [25], [27]), of D.M. Eidus ([12], [13]; see also [30]) and of R. Phillips [22] (see also [20]). The key result is a division theorem for $A_{0}$ (see theorem 4.1). An another application of the division theorem we give in this article is a result concerning decreasing properties of eigenfunctions of $A$ associated with positive eigenvalues (see theorem 5.5).

In fact we adapt to linear elasticity the method that has been used in [6] for sound propagation in stratified media (see also [29]).

The starting point is the explicit knowledge of the modes for an homogeneous isotropic halfspace with a free boundary. We call them the unperturbed modes. These modes are the P-plane waves and their reflections, the SV and $\mathrm{SH}$ plane waves and their reflections and the Rayleigh surface waves (see [1], [4], [5], [11], [26]). They generate the solutions with finite energy of the wave equailion for elastic waves in an homogeneous isotropic half space with a fret boundary.

In this article the main results concern the selfadjoint operator $A_{0}$. The operator $A$ includes only the case of a free boundary condition and solid inclusions. More general examples including rigid and fluid inclusions will be considered in a next paper.

The results have been announced in [7] and [8]. But note that notations used in this article are different from these used in [7] and [8].

In a next paper distorded modes for $A$ will be deduced from the limiting absorption principle and the scattering theory will be developped including the existence and the completness of the wave operators and representation theorems for the scattering matrix and the scattering amplitudes by using the distorded modes. 
The article is organized as follows.

In the second section the selfadjoint operator $A$ is defined and a qualitative description of the main results is given.

In the third section the spectral analysis of the selfadjoint operator $\mathrm{A}_{0}$ is given. The set of the unperturbed modes appear as a complete set of generalized eigenfunctions of this selfadjoint operator. The limiting absorption principle in appropriate Hilbert spaces is given for the resolvent of $A_{0}$. Both of the half spaces $\mathbf{R}_{+}^{2}=\left\{\left(\mathrm{x}_{1}, \mathrm{x}_{2}\right) \in \mathbb{R}^{2}, \mathrm{x}_{2}>0\right\}$ and $\mathbb{R}_{+}^{3}=\left\{\left(\mathrm{x}_{1}, \mathrm{x}_{2}, \mathrm{x}_{3}\right) \in \mathbb{R}^{3}, \mathrm{x}_{3}>0\right\}$ are considered.

In the fourth section we prove the basic division theorem for $A_{0}$.

Finally in the fifth section we give two different proofs of the limiting absorption principle in appropriate Hilbert spaces for the resolvent of $A$. We then deduce some spectral properties of $A$ and give a result concerning decreasing properties of eigenfunctions of $A$ associated with strictly positive eigenvalues.

Acknowledgments

We would like to thank Prof. J. Geymonat for helpful discussions. 
2. LINEAR ELASTICITY IN A PERTURBED ISOTROPIC HALF SPACE WITH A FREE BOUNDARY AND THE MAIN RESULT

Let $\mathbb{R}_{+}^{3}=\left\{\mathrm{x}=\left(\mathrm{x}_{1}, \mathrm{x}_{2}, \mathrm{x}_{3}\right) \in \mathbb{R}^{3} ; \mathrm{x}_{3}>0\right\}$ and $|\mathrm{x}|$ be the euclidian norm of $x$. Let $L$ be a fixed strictly positive real number.

$\Omega$ denotes an open set of $\mathbb{R}^{3}$ such that $\Omega \cap\left\{\mathrm{x} \in \mathbb{R}^{3} ;|\mathrm{x}|>\mathrm{L}\right\}=$ $\left\{\mathbf{x} \in \mathbb{R}_{+}^{3} ;|\mathbf{x}|>\mathrm{L}\right\}$. Let $\bar{\Omega}$ be the closure of $\Omega$.

We assume that $\Omega$ satisfies the cone condition, i.e., there exist positive constants $\alpha, \mathrm{h}$ such that for any $\mathrm{x} \in \Omega$ one can construct a right spherical cone $v_{x}$ with vertex $x$, opening $\alpha$ and height $h$ such that it lies in $\Omega$.

$\Omega$ can be the exterior in $\mathbb{R}_{+}^{3}$ of any compact set $\mathrm{K}$ whose interior is empty as for example a slit, a penny-shaped crack and, more generally, a compact flat crack of arbitrary shape such that the cone condition is satisfied.

We assume that an inhomogeneous isotropic solid occupies the domain $\Omega$. The propagation of elastic waves is such a solid is governed by the following functions of $x \in \bar{\Omega}$

(2.1) $\rho(x)$ the equilibrium density of the solid

(2.2) $\lambda(x)$ and $\mu(x)$ the Lamé functions.

We assume that

$$
0<\mathrm{m} \leq \lambda(\mathrm{x}) \quad(\text { resp. } \mu(\mathrm{x}), \rho(\mathrm{x})) \leq \mathrm{M} \text { for a.e. } \mathrm{x} \text { in } \bar{\Omega}
$$

and

$$
\lambda(x)=\lambda_{0}, \mu(x)=\mu_{0} \quad \text { and } \rho(x)=\rho_{0} \text { for }|x|>R \text {. }
$$


The state of the elastic field in the solid is determined by

(2.4) $u(x ; t)=\left(u_{1}(x, t), u_{2}(x, t), u_{3}(x, t)\right) \in \mathbb{R}^{3}$, the displacement field of the solid at time $t$ and position $x$

and

(2.5) $\sigma_{i j}(x, t), i, j=1,2,3$, the symmetric stress tensor field of the solid at time $t$ and position $x$.

The equations satisfied by the elastic field in the isotropic solid are

$\cdot(2.6) \quad \sigma_{i j}(u)=\lambda(\cdot)(\nabla \cdot u) \delta_{i j}+2 \mu(\cdot) \varepsilon_{i j}(u)$

where

(2.7) $\varepsilon_{i j}(u)=\frac{1}{2}\left(\frac{\partial u_{i}}{\partial x_{j}}+\frac{\partial u_{j}}{\partial x_{i}}\right)$

and

(2.8) $\quad \frac{\partial^{2} u_{i}}{\partial t^{2}}=\frac{1}{\rho(.)} \frac{\partial \sigma_{i j}}{\partial x_{j}}(u)$.

Thus the displacement field $u$ satisfies the following equations :

$$
\frac{\partial^{2} u_{i}}{\partial t^{2}}-\frac{1}{\rho(.)} \frac{\partial}{\partial x_{j}}\left(\lambda(.)(\nabla \cdot u) \delta_{i j}+2 \mu(.) \varepsilon_{i j}(u)\right)=0 \text {. }
$$

The wave equation (2.9) must be supplemented by boundary conditions at the boundary. $\partial \Omega$ of the solid. In the case of free boundaries the normal component of the stress must vanish at the boundary. Thus

$$
\sigma_{i j}(u) v_{j \mid \partial \Omega}=0
$$


where $\nu$ is the exterior normal at point $x \in \partial \Omega$. We could consider rigid boundaries too, for which the displacement must vanish at the boundary.

In this article we restrict ourselves to free boundaries. It is one of the most important cases in pratical situations. Other cases will be dealt with along the same line in the next article.

With solutions to (2.9) and (2.10) with finite energy are usually associated a Hilbert space and a selfadjoint operator as follows.

Let

$$
\begin{aligned}
& \text { (2.11) } \quad(\mathscr{A})_{i}=-\frac{1}{\rho(\cdot)} \frac{\partial}{\partial x_{j}} \sigma_{i j}(u) \text { and } \\
& L^{2}\left(\Omega, \mathbb{A}, \mathbb{C}^{3}\right)=\left\{u \in L^{2}\left(\Omega, \mathbb{C}^{3}\right) ; \mathbb{A} u \in L^{2}\left(\Omega, \mathbb{C}^{3}\right)\right\}
\end{aligned}
$$

$\mathrm{H}^{\mathrm{m}}\left(\Omega, \mathbb{C}^{3}\right)$ denotes the usual Sobolev space. $u$ in $\mathrm{H}^{1}\left(\Omega, \mathbb{C}^{3}\right)$ in $\mathrm{L}^{2}\left(\Omega, \mathcal{A}, \mathbf{c}^{3}\right)$ is said to satisfy the generalized free boundary condition if and only if one has

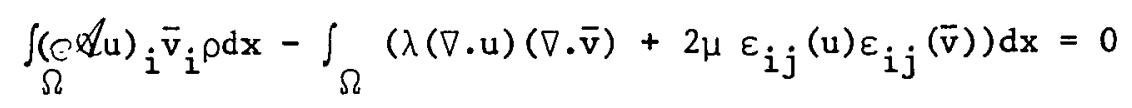

for every $v$ in $H^{\jmath}\left(\Omega, \mathbb{C}^{3}\right)$.

The following operator $(D(A), A)$ in $L^{2}\left(\Omega, C^{3}, \rho(x) d x\right)$ :

(2.13) $\quad \mathrm{D}(\dot{A})=\left\{\mathrm{u} \in \mathrm{H}^{1}\left(\Omega, \mathbb{C}^{3}\right) \cap \mathrm{L}^{2}\left(\Omega, \mathcal{A}, \mathbb{C}^{3}\right) ; \mathrm{u}\right.$ satisfies the generalized free boundary condition\}

(2.14) $\quad A u=\notin \mathbb{u}, u \in D(A)$

is a positive selfadjoint operator. 
This is a consequence of the Korn's inequality (cf. [10], [15]) which follows from the fact that $\Omega$ satisfies the cone condition. The proof of the selfadjointness of $A$ can be found in [19] or in [30]. If $\partial \Omega$ is smooth, then each $u$ in $D(A)$ belongs to $H^{2}\left(\Omega, \mathbb{c}^{3}\right)$ (see [21], p. 222) and (2.10) is satisfied in the usual sense.

Let us consider a homogeneous isotropic half space $\mathbb{R}_{+}^{3}$ with a free boundary and with the density $\rho_{0}$ and the Lamé constants $\lambda_{0}$ and $\mu_{0}$. We then define the positive selfadjoint operator $\left(D\left(A_{0}\right), A_{0}\right)$ in $L^{2}\left(R_{+}^{3}, \mathbb{C}^{3}, \rho_{0} \mathrm{dx}\right)$ as follows (cf. [10], [19], [30], [21, p. 222])

$$
\begin{aligned}
D\left(A_{0}\right)=\left\{u \in H^{2}\left(R_{+}^{3}, \mathbb{C}^{3}\right) ;\right. & \left.\varepsilon_{13}(u)\right|_{x_{3}}=0=\left.\varepsilon_{23}(u)\right|_{x_{3}=0}= \\
& \left.=\lambda_{0} \nabla \cdot u+\left.2 \mu_{0} \varepsilon_{33}(u)\right|_{x_{3}=0}=0\right\}
\end{aligned}
$$

(2.16) $\quad A_{0} u=-\frac{\lambda_{0}+\mu_{0}}{\rho_{0}} \nabla(\nabla . u)-\frac{\mu_{0}}{\rho_{0}} \Delta u, \quad u \in D\left(A_{0}\right)$,

$A$ is considered as a perturbation of $A_{0}$. We restrict ourselves to perturbations with compact support because it is always the case for applications. One could also consider short range perturbations of $A_{0}$ as in [6] and [29]. $A^{1 / 2}$ (resp. $A_{0}^{1 / 2}$ ) denotes the square root of $A$ (resp. $\left.A_{0}\right)$. The solution of $(2.9)$ and $(2.10)$ satisfying the Cauchy conditions :

(2.17) $u(x, 0)=f(x)$ and $\frac{\partial u}{\partial t}(x, 0)=g(x)$

is given by

(2.18) $u(x, t)=\left(\cos A^{1 / 2} t f\right)(x)+\left(\frac{\sin A^{1 / 2} t}{A^{1 / 2}} g\right)(x)$

if $f$ belongs to $D\left(A^{l / 2}\right)$ and $g$ to $L^{2}\left(\Omega, C^{3}, \rho(x) d x\right)$. 
In fact $u(., t)$ is in the class $C^{1}\left(\mathbb{R}, L^{2}\left(\Omega, \mathbb{C}^{3}, \rho(x) d x\right)\right) \cap C^{0}\left(\mathbb{R}, D\left(A^{1 / 2}\right)\right)$ and satisfies the wave equation

$$
\frac{\mathrm{d}^{2} \mathrm{u}}{\mathrm{dt}^{2}}+\mathrm{Au}=0 \text { for all } \mathrm{t} \in \mathbb{R}
$$

and

(2.20) $u(0)=f$ and $\frac{d u}{d t}(0)=g$.

In this case $u(., t)$ is called a solution with finite energy (cf. [30]).

Let $E(K, u, t)$ be the restriction of the energy of $u$ to a measurable subset $\mathrm{K}$ of $\Omega$ :

$$
\begin{aligned}
E(K, u, t)=\frac{1}{2} \sum_{i=1}^{3} \int_{K}\left|\frac{\partial u_{i}}{\partial t}\right|^{2} \rho(x) d x & +\sum_{i, j=1}^{3} \int_{K}\left(\lambda(x)|\nabla \cdot u|^{2}\right. \\
& \left.+2 \mu(x)\left|\varepsilon_{i j}(u)\right|^{2}\right) d x
\end{aligned}
$$

$E(u, t)$ denotes the energy of $u$, i.e., $E\left(\mathbb{R}_{+}^{3}, u, t\right)$.

A study of further properties of $u$ is based on a knowledge of the spectral properties of $A$.

It will be shown in section 3 that there exists a complete set of generalized eigenfunctions $\psi_{\mathrm{P}}, \psi_{\mathrm{SV}}, \psi_{\mathrm{SV}}^{0}, \psi_{\mathrm{SH}}$ and $\psi_{\mathrm{R}}$ for $\mathrm{A}_{0}$. Thus, in this case, the solution $u$, constructed from $A_{0}$, can be written as a sum of wave-packets of P-plane waves and their reflections, of s-plane waves and their reflections and of Rayleigh surface waves.

The spectrum of $A_{0}$ is $[0, \infty)$ and $A_{0}$ is an absolutely continuous operator (see [19] for definitions). This will be proved in section 3 . 
Every solution $u$ with finite energy associated with $A_{0}$ is a transient state in the sense that, for every compact set $k$ in $\mathbb{R}_{+}^{3}, E(k, u, t)$ tends to zero when $t$ tends to $+\infty$.

The spectrum of $A$ is $[0, \infty)$. Its structure is more complicated. Its continuous spectrum is also $[0, \infty)$ but $A$ can have positive eigenvalues embedded in the continuous spectrum. Eigenvalues can accumulate only at 0 and $+\infty$. A has no continuous singular spectrum. All these results will be proved in section 5 and in the next article.

The main result of this article is the proof of the limiting absorption principle for A.

More precisely, let $R(z)$ (resp. $R_{0}(z)$ ) be the resolvent of $A$ (resp. $\mathrm{A}_{0}$ ) :

$$
R(z)=(A-z)^{-1} \quad\left(\text { resp. } R_{0}(z)=\left(A_{0}-z\right)^{-1}\right)
$$

Then the limiting absorption principle states that $R_{0}(z)$ and $R(z)$ have a limit as bounded operators in appropriate Hilbert spaces when $z$ tends to $\omega^{2}>0$ with a positive (or negative) imaginary part and when $\omega^{2}$ is not an eigenvalue of $A$ in the case of $R(z)$.

Rigourous statements will be given in sections 3 and 5 . 
3. THE SPECTRAL ANALYSIS OF $A_{0}$ IN $\mathbb{R}_{+}^{3}$ AND $\mathbb{R}_{+}^{2}$ AND THE LIMITING ABSORPTION PRINCIPLE

The spectral analysis of $A_{0}$ in $\mathbb{R}_{+}^{2}$ has been studied in [28]. J.R. Schulenberger has transformed the $2 \times 2$ second order system of 1 inear elasticity into a $5 \times 5$ first order system. This approach introduces static solutions with no physical meaning. Here we consider the usual equations (see [1], [4], [5], [11]) and we follow the method developped in [15], [16], [17], [31] and [32] in order to analyse the spectral properties of $A_{0}$ and to construct a spectral representation for it. In this approach $A_{0}$ in $\mathbb{R}_{+}^{3}$ (resp. $\mathbb{R}_{+}^{2}$ ) is considered as unitarly equivalent to a direct integral of a field of $3 \times 3$ systems (resp. $2 \times 2$ systems) of Sturm-Liouville differential operators because of the symmetry of the problem. It is then sufficient to analyse the spectral properties of every $3 \times 3$ system (resp. $2 \times 2$ system) of Sturm-Liouville differential operators. We then construct a spectral representation for $\mathrm{A}_{0}$ from which we deduce the limiting absorption principle. Some details are given for $A_{0}$ in $\mathbb{R}_{+}^{3}$. We only give the results for $A_{0}$ in $\mathbb{R}_{+}^{2}$ (see remark 3 ).

Let $p=\left(p_{1}, p_{2}\right) \in \mathbb{R}^{2}$ be the conjuguate variables of $\left(x_{1}, x_{2}\right)$ and let $\$$ be the partial Fourier transform with respect to $\left(x_{1}, x_{2}\right)$ : (3.1) $\left(\sigma_{\mathrm{u}}\right)\left(\mathrm{p}, \mathrm{x}_{3}\right)=\frac{1}{2 \pi}$ L.i.m $\int_{\mathbb{R}^{2}} \mathrm{e}^{-\mathrm{i}\left(\mathrm{p}_{1} \mathrm{x}_{1}+\mathrm{p}_{2} \mathrm{x}_{2}\right)} \mathrm{u}\left(\mathrm{x}_{1}, \mathrm{x}_{2}, \mathrm{x}_{3}\right) \mathrm{dx} \mathrm{x}_{1} \mathrm{dx_{2 }}$ for $u$ in $L^{2}\left(\mathbb{R}_{+}^{3}, \mathbb{C}^{3}, \rho_{0} \mathrm{dx}\right)$

Let

$$
D\left(\hat{A_{0}}\right)=\varnothing D\left(A_{0}\right)
$$

(3.3) $\quad \hat{A}_{0}=\mathscr{Q}_{A_{0}} \mathscr{P}^{-1}$ 
$\hat{A}_{0}$ is a positive selfadjoint operator in $L^{2}\left(\mathbb{R}_{+}^{3}, \mathbb{C}^{3}, \rho_{0} \mathrm{dx}\right)$ unitarly equivalent to $A_{0}$.

Let

$$
\begin{aligned}
D\left(\hat{A}_{0}\left(\mathrm{p}_{1}, \mathrm{P}_{2}\right)\right)=\left\{\mathrm{u} \in \mathrm{H}^{2}\left(\mathbb{R}_{+}, \mathbb{C}^{3}\right) ;\right. & \frac{\mathrm{du}}{\mathrm{dx_{3 }}}(0)+\mathrm{ip}_{1} \mathrm{u}_{3}(0)=0, \\
& \frac{\mathrm{du}}{\mathrm{dx}_{3}}(0)+i \mathrm{p}_{2} \mathrm{u}_{3}(0)=0, \\
& \left(\lambda_{0}+2 \mu_{0}\right) \frac{\mathrm{du}_{3}}{\mathrm{dx}_{3}}(0)+i \lambda_{0} \mathrm{p}_{1} \mathrm{u}_{1}(0) \\
& \left.+i \lambda_{0} \mathrm{p}_{2} \mathrm{u}_{2}(0)=0\right\}
\end{aligned}
$$

and

$$
\hat{A}_{0}\left(p_{1}, p_{2}\right)\left(\begin{array}{l}
u_{1} \\
u_{2} \\
u_{3}
\end{array}\right)\left(x_{3}\right)=
$$

$\rho_{0}^{-1}\left(\begin{array}{lll}-\mu_{0} \frac{d^{2}}{d x_{3}^{2}}+\left(\lambda_{0}+2 \mu_{0}\right) p_{1}^{2}+\mu_{0} p_{2}^{2}, & \left(\lambda_{0}+\mu_{0}\right) p_{1} p_{2} & ,-i p_{1}\left(\lambda_{0}+\mu_{0}\right) \frac{d}{d x_{3}} \\ \left(\lambda_{0}+\mu_{0}\right) p_{1} p_{2} & ,-\mu_{0} \frac{d^{2}}{d x_{3}^{2}}+\mu_{0} p_{1}^{2}+\left(\lambda_{0}+2 \mu_{0}\right) p_{2}^{2},-i p_{2}\left(\lambda_{0}+\mu_{0}\right) \frac{d}{d x_{3}} \\ -i p_{1}\left(\lambda_{0}+\mu_{0}\right) \frac{d}{d x_{3}} & ,-i p_{2}\left(\lambda_{0}+\mu_{0}\right) \frac{d}{d x_{3}} & ,-\left(\lambda_{0}+2 \mu_{0}\right) \frac{d^{2}}{d x_{3}^{2}}+\mu_{0}|p|^{2}\end{array}\right)\left(\begin{array}{l}u_{1} \\ u_{2} \\ u_{3}\end{array}\right)\left(x_{3}\right)$

for $u$ in $D\left(\hat{A}_{0}\left(p_{1}, p_{2}\right)\right)$.

For each $\mathrm{p}=\left(\mathrm{p}_{1}, \mathrm{p}_{2}\right) \in \mathbb{R}^{2}, \hat{\mathrm{A}}_{0}\left(\mathrm{p}_{1}, \mathrm{p}_{2}\right)$ with its domain $\mathrm{D}\left(\hat{\mathrm{A}}_{0}\left(\mathrm{p}_{1}, \mathrm{p}_{2}\right)\right)$ is a positive selfadjoint operator in $L^{2}\left(\mathbb{R}_{+}, \mathbb{C}^{3}, \rho_{0}\right.$ dy) (see [17]). 
To get the spectral analysis of $A_{0}$, the first remark to be made is that $\hat{\mathrm{A}}_{0}$ is the direct integral of the field $\left(\hat{\mathrm{A}}_{0}\left(\mathrm{p}_{1}, \mathrm{p}_{2}\right)\right)\left(\mathrm{p}_{1}, \mathrm{p}_{2}\right) \in \mathbb{R}^{2}$ of
selfadjoint operators:

(3.6) $\quad \hat{A}_{0}=\int_{\mathbb{R}^{2}}^{\oplus} \hat{A}_{0}\left(p_{1}, p_{2}\right) d p_{1} d p_{2}$

(see $[15],[17],[32])$.

The second remark to be made is that the isotropic half space is invariant with respect to any rotation around the $x_{3}$-axis. Using this property we show that for, every $\left(p_{1}, p_{2}\right) \neq 0, \hat{A}_{0}\left(p_{1}, p_{2}\right)$ is unitarily equivalent to a simpler operator whose study is easier.

For every $p \neq 0$ we consider the following $3 \times 3$ matrix :

$$
\mathrm{U}\left(\mathrm{p}_{1}, \mathrm{p}_{2}\right)=\frac{1}{|\mathrm{p}|}\left(\begin{array}{ccc}
\mathrm{p}_{1} & -\mathrm{p}_{2} & 0 \\
\mathrm{p}_{2} & \mathrm{p}_{1} & 0 \\
0 & 0 & |\mathrm{p}|
\end{array}\right)
$$

where

$$
\text { (3.8) } \quad|p|=\left(p_{1}^{2}+p_{2}^{2}\right)^{1 / 2} \text {. }
$$

We have

$$
\mathrm{U}\left(\mathrm{p}_{1}, \mathrm{p}_{2}\right)^{-1}=\mathrm{U}\left(\mathrm{p}_{1},-\mathrm{p}_{2}\right)
$$

and

\section{Proposition 3.1}

For every $\mathrm{p}=\left(\mathrm{p}_{1}, \mathrm{p}_{2}\right) \neq 0$ one has

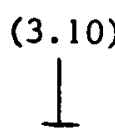

$$
\hat{A}_{0}\left(p_{1}, p_{2}\right)=U\left(p_{1}, p_{2}\right) \hat{A}_{0}(|p|, 0) U\left(p_{1},-p_{2}\right)
$$


As it is shown in [17] $\hat{A}(|p|, 0)$ is a direct sum of two selfadjoint operators respectively denoted by $B_{1}(|p|)$ and $B_{2}(|p|)$ and defined as follows :

$$
D\left(B_{1}(|p|)\right)=\left\{\left(\begin{array}{l}
u_{1} \\
u_{2}
\end{array}\right) \in H^{2}\left(\mathbb{R}_{+}, \mathbb{C}^{2}\right) ; \frac{d u_{1}}{d x_{3}}(0)+i|p| u_{3}(0)=\right.
$$

(3.11)

$$
\left.=\left(\lambda_{0}+2 \mu_{0}\right) \frac{\mathrm{du}_{3}}{\mathrm{dx}_{3}}(0)+\mathrm{i}|\mathrm{p}| \lambda_{0} \mathrm{u}_{1}(0)=0\right\}
$$

and

(3.12) $\quad B_{1}(|p|)\left(\begin{array}{l}u_{1} \\ u_{3}\end{array}\right)=\rho_{0}^{-1}$

$$
\left[\begin{array}{c}
-\mu_{0} \frac{d^{2}}{d x_{3}^{2}}+\left(\lambda_{0}+2 \mu_{0}\right)|p|^{2},-i|p|\left(\lambda_{0}+\mu_{0}\right) \frac{d}{d x_{3}} \\
-i|p|\left(\lambda_{0}+\mu_{0}\right) \frac{d}{d x_{3}} \\
u_{3}
\end{array}\right]\left[\begin{array}{c}
u_{1} \\
\\
u_{3}
\end{array}\right]
$$

$B_{1}(|p|)$ is a positive selfadjoint operator in $L^{2}\left(\mathbb{R}_{+}, \mathbb{C}^{2}, \rho_{0} d x_{3}\right)$,

(3.13) $\quad D\left(B_{2}(|p|)\right)^{-}=\left\{u_{2} \in H^{2}\left(\mathbb{R}_{+}\right) ; \frac{d u_{2}}{d x_{3}}(0)=0\right\}$

(3.14) $\quad B_{2}(|p|) u_{2}=-\mu_{0} \rho_{0}^{-1} \frac{d^{2} u_{2}}{{d x_{3}^{2}}_{3}}+\mu_{0} \rho_{0}^{-1}|p|^{2} u_{2}$

$B_{2}(|p|)$ is a positive selfadjoint operator in $L^{2}\left(\mathbb{R}_{+}, \mathbb{C}, \rho_{0} d_{x_{3}}\right)$.

In fact we have

Proposition 3.2

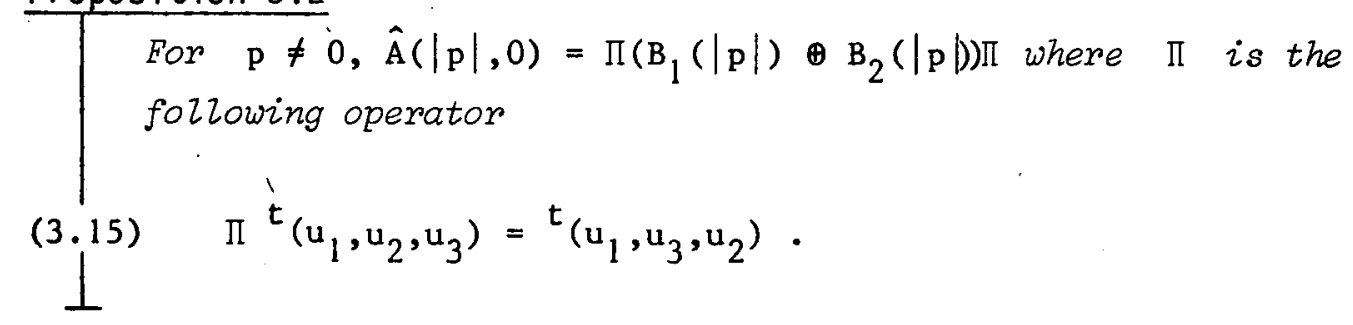


As well known $\mathrm{B}_{2}(|\mathrm{p}|)$ is an absolutely continuous operator whose spectrum is $\left[\mu_{0} / \rho_{0}|p|^{2}, \infty\right)$. It is not the case for $B_{1}(|p|)$. In fact we have the following result.

\section{Proposition 3.3}

For every $\mathrm{p} \neq 0, \mathrm{~B}_{1}(|\mathrm{p}|)$ has an unique simple eigenvalue $\mathrm{c}_{\mathrm{R}}^{2}|\mathrm{p}|^{2}$ such that $0<\mathrm{c}_{\mathrm{R}}^{2}|\mathrm{p}|^{2}<\left(\mu_{0} / \rho_{0}\right)|\mathrm{p}|^{2}$.

$c_{R}>0$ is the unique solution in $\left(0,\left(\mu_{0} / \rho_{0}\right)^{1 / 2}\right)$ to the following implicit equation

$$
\left(1-\frac{\alpha^{2} \rho_{0}}{2 \mu_{0}}\right)^{2}-\left(1-\frac{\alpha^{2} \rho_{0}}{\mu_{0}}\right)^{1 / 2}\left(1-\frac{\alpha^{2} \rho_{0}}{\lambda_{0}+2 \mu_{0}}\right)^{1 / 2}=0 .
$$

For a proof of proposition 3.3 see [17]. This unique eigenvalue for $B_{1}(|p|)$ is the origin of the Rayleigh surface wave and $c_{R}$ is its, speed.

The spectral analysis of $B_{1}(|p|)$ and $B_{2}(|p|)$ is based on a formulation of the Weyl-Kodaira theory due to N. Dunford and J.T. Schwartz [9; chapter XIII]. However the theorems given in [9; chapter XIII] are not directely applicable to $B_{1}(|p|)$. One verifies that the DunfordSchwartz theorems can be easily generalized to selfadjoint operators associated with $2 \times 2$ systems of Sturm-Liouville operators in order to get the spectral analysis and spectral representation of these selfadjoint realizations. In this article we only give the results.

Finally, from the spectral analysis of $B_{1}(|p|)$ and $B_{2}(|p|)$, from propositions $3.3,3.2$ and 3.1 and from (3.2) and (3.6) we obtain the spectral analysis of $A_{0}$. Expansions in terms of generalized eigenfunctions for $A_{0}$ are given. These generalized eigenfunctions are exactly the same as those used by physicists and engineers. They are P-plane waves and their reflections, S-plane waves and their reflections and the Rayleigh surface wave. 
Let

(3.17) $\quad c_{P}^{2}=\frac{\lambda_{0}+2 \mu_{0}}{\rho_{0}}$ and $\quad c_{S}^{2}=\frac{\mu_{0}}{\rho_{0}}$

$$
E=\left\{(p, \omega) \in \mathbb{R}_{+}^{3} ; \omega>c_{p}|p|\right\}
$$

(3.18) $\quad E_{S V}^{0}=\left\{(p, \omega) \in \mathbb{R}_{+}^{3} ; c_{S}|p|<\omega<c_{P}|p|\right\}$

$$
\begin{aligned}
& E_{S H}=\left\{(p, \omega) \in \mathbb{R}_{+}^{3} ; c_{S}|p|<\omega\right\} \\
& \xi_{P}\left(\omega^{2}\right)=\left(\omega^{2} / c_{P}^{2}-|p|^{2}\right)^{1 / 2} \text { for }(p, \omega) \in E \text { and }
\end{aligned}
$$

(3.19)

$$
\xi_{S}\left(\omega^{2}\right)=\left(\omega^{2} / c^{2}-|p|^{2}\right)^{1 / 2} \text { for }(p, \omega) \in E_{S H}
$$

(3.20)

$$
\xi_{p}^{\prime}\left(\omega^{2}\right)=\left(|p|^{2}-\omega^{2} / c_{P}^{2}\right)^{1 / 2} \text { for } \omega<c_{p}|p|
$$

\section{Definition 3.5}

(i) For every $\mathrm{x} \in \mathbb{R}_{+}^{3}$ and for every $(\mathrm{p}, \omega) \in \mathrm{E}$ let

$$
\psi_{\mathrm{P}}(\mathrm{x} ; \mathrm{p}, \omega)=\frac{1}{(2 \pi)^{3 / 2}} \frac{e^{i\left(\mathrm{p}_{1} \mathrm{x}_{1}+\mathrm{p}_{2} \mathrm{x}_{2}\right)}}{\left(\omega \rho_{0} \xi_{\mathrm{p}}\left(\omega^{2}\right)\right)^{1 / 2}}\left[\mathrm{e}^{-i \xi_{\mathrm{p}}\left(\omega^{2}\right) \mathrm{x}_{3}} \mathrm{t}_{\left(\mathrm{p}_{1}, \mathrm{p}_{2},-\xi_{\mathrm{p}}\left(\omega^{2}\right)\right)}\right.
$$

$$
+\frac{4|p|\left(\omega^{2} / c_{s}^{2}-2|p|^{2}\right) \xi_{p}\left(\omega^{2}\right)}{\left(\omega^{2} / c_{S}^{2}-2|p|^{2}\right)^{2}+4|p|^{2} \xi_{p}\left(\omega^{2}\right) \xi_{S}\left(\omega^{2}\right)}
$$

$$
\begin{gathered}
\left.e^{i \xi_{S}\left(\omega^{2}\right) x_{3}}{ }_{t}^{t_{T p} p_{1}} \xi_{S}\left(\omega^{2}\right), \frac{p_{2}}{|p|} \xi_{S}\left(\omega^{2}\right),-|p|\right) \\
-\frac{\left(\omega^{2} / c_{S}^{2}-2|p|^{2}\right)^{2}-4|p|^{2} \xi_{p}\left(\omega^{2}\right) \xi_{S}\left(\omega^{2}\right)}{\left(\omega^{2} / c_{S}^{2}-2|p|^{2}\right)^{2}+4|p|^{2} \xi_{P}\left(\omega^{2}\right) \xi_{S}\left(\omega^{2}\right)} \\
e^{i \xi_{P}\left(\omega^{2}\right) x_{3} \quad t}{ }_{\left.\left(p_{1}, p_{2}, \xi_{P}\left(\omega^{2}\right)\right)\right]}
\end{gathered}
$$


(ii) For every $\mathrm{x} \in \mathbf{R}_{+}^{3}$ and for every $(\mathrm{p}, \omega) \in \mathrm{E}$ let

$$
\begin{aligned}
& \psi_{S V}(x ; p, \omega)=\frac{1}{(2 \pi)^{3 / 2}} \frac{e^{i\left(p_{1} x_{1}+p_{2} x_{2}\right)}}{\left(\omega \rho_{0} \xi_{S}\left(\omega^{2}\right)\right)^{1 / 2}}\left[e^{-i \xi_{S}\left(\omega^{2}\right) x_{3}} \tau \frac{p_{1}}{|p|^{\xi} S}\left(\omega^{2}\right) \cdot \frac{p_{2}}{\mid p T^{\xi} S}\left(\omega^{2}\right), p \mid\right) \\
& +\frac{\left(\omega^{2} / c^{2} s^{-2}|p|^{2}\right)^{2}-4|p|^{2} \xi_{P}\left(\omega^{2}\right) \xi_{S}\left(\omega^{2}\right)}{\left(\omega^{2} / c^{2}-2|p|^{2}\right)^{2}+4|p|^{2} \xi_{P}\left(\omega^{2}\right) \xi_{S}\left(\omega^{2}\right)}
\end{aligned}
$$

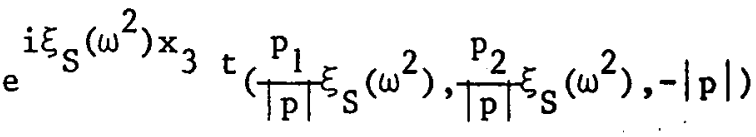

$$
\begin{aligned}
& +\frac{4|p|\left(\omega^{2} / c_{S}^{2}-2|p|^{2}\right) \xi_{S}\left(\omega^{2}\right)}{\left(\omega / c_{S}^{2}-2|p|^{2}\right)^{2}+4|p|^{2} \xi_{p}\left(\omega^{2}\right) \xi_{S}\left(\omega^{2}\right)}
\end{aligned}
$$

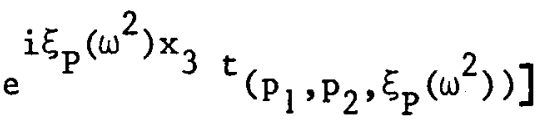

(iii) For every $\mathrm{x} \in \mathbb{R}_{+}^{3}$ and for every $(\mathrm{p}, \mathrm{w}) \in \mathrm{E}_{\mathrm{SV}}^{0}$ let

$$
\begin{aligned}
& \psi_{S V}^{0}(x ; p, \omega)=\frac{1}{(2 \pi \mathrm{T})^{3 / 2}} \frac{e^{i\left(p_{1} x_{1}+p_{2} x_{2}\right)}}{\left(\omega \rho_{0} \xi_{S}\left(\omega^{2}\right)\right)^{1 / 2}}
\end{aligned}
$$

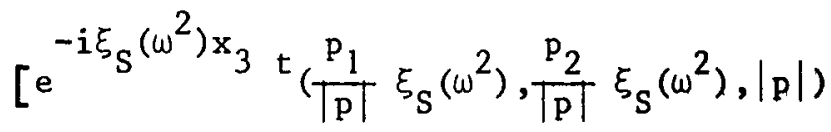

$$
\begin{aligned}
& +\frac{\left(\omega^{2} / c_{s}^{2}-2|p|^{2}\right)^{2}-4 i|p|^{2} \xi_{p}^{\prime}\left(\omega^{2}\right) \xi_{S}\left(\omega^{2}\right)}{\left(\omega^{2} / c_{S}^{2}-2|p|^{2}\right)^{2}+4 i|p|^{2} \xi_{p}^{\prime}\left(\omega^{2}\right) \xi_{S}\left(\omega^{2}\right)} \\
& \left.e^{i \xi_{S}\left(\omega^{2}\right) x_{3}} t \frac{p_{1}}{|p|} \xi_{S}\left(\omega^{2}\right), \frac{p_{2}}{|p|} \xi_{S}\left(\omega^{2}\right),-|p|\right)
\end{aligned}
$$




$$
\begin{array}{r}
+\frac{4|p|\left(\omega^{2} / c_{s}^{2}-2|p|^{2}\right) \xi_{s}\left(\omega^{2}\right)}{\left(\omega^{2} / c^{2} s^{\left.-2|p|^{2}\right)^{2}+4 i|p|^{2} \xi_{p}^{\prime}\left(\omega^{2}\right) \xi_{S}\left(\omega^{2}\right)}\right.} \\
e^{\left.\left.-\xi_{p}^{\prime}\left(\omega^{2}\right) x_{3} t_{\left(p_{1}\right.}, p_{2}, i \xi_{p}^{\prime}\left(\omega^{2}\right)\right)\right]}
\end{array}
$$

(iv) For every $\mathrm{x} \in \mathbb{R}_{+}^{3}$ and for every $(\mathrm{p}, w) \in \mathrm{E}_{\mathrm{SH}}$ let

$$
\psi_{S H}(x ; p, \omega)=\frac{1}{c_{s} \pi^{3 / 2}}\left(\frac{\omega}{2 \rho_{0} \xi_{S}\left(\omega^{2}\right)}\right)^{1 / 2} e^{i\left(p_{1} x_{1},+p_{2} x_{2}\right)} \cos \xi_{S}\left(\omega^{2}\right) x_{3}
$$

(3.24)

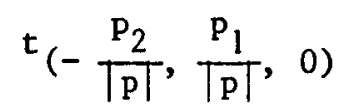

(v) For every $\mathrm{x} \in \mathbb{R}_{+}^{3}$ and for every $\mathrm{p} \in \mathbf{R}^{2}$ let

$$
\begin{gathered}
\Psi_{R}(x ; p)=\frac{c}{2 \pi} e^{i\left(p_{1} x_{1}+p_{2} x_{2}\right)}|p|^{1 / 2}\left[\left(2-\left(c_{R} / c_{S}\right){ }^{2}\right) e^{-|p|\left(1-\left(c_{R} / c_{P}\right)^{2}\right)^{1 / 2} x_{3}}\right. \\
\left.{ }_{\left(i \frac{p_{1}}{T p} T\right.}, i \frac{p_{2}}{|p|},-\left(1-\left(c_{R} / c_{p}\right)^{2}\right)^{1 / 2}\right)
\end{gathered}
$$

$$
\begin{aligned}
& \quad 2\left(1-\left(c_{R} / c_{P}\right)^{2}\right)^{1 / 2} e^{-|p|\left(1-\left(c_{R} / c_{S}\right)^{2}\right)^{1 / 2} x_{3}} \\
& \left.\left.{ }^{t}{ }_{(i} \frac{p_{1}^{1}}{T T^{-}}\left(1-\left(c_{R} / c_{S}\right)^{2}\right)^{1 / 2}, i \frac{p_{2}}{T p T^{-}}\left(1-\left(c_{R} / c_{S}^{2}\right)^{2}\right)^{1 / 2},-1\right)\right]
\end{aligned}
$$

c is a strictly positive constant such that

(3.26) $4 \pi^{2} \int_{0}^{\infty}\left(\left|\psi_{\mathrm{R} 1}(\mathrm{x} ; \mathrm{p})\right|^{2}+\left|\psi_{\mathrm{R} 2}(\mathrm{x} ; \mathrm{p})\right|^{2}+\left|\psi_{\mathrm{R} 3}(\mathrm{x} ; \mathrm{p})\right|^{2}\right) \mathrm{p}_{0} \mathrm{~d} \mathrm{x}_{3}=1$.

$\psi_{\mathrm{P}}, \psi_{\mathrm{SV}}, \psi_{\mathrm{SV}}^{0}, \psi_{\mathrm{SH}}$ and $\psi_{\mathrm{R}}$ are generalized eigenfunctions for $\mathrm{A}_{0}$.

We then have the following theorem 
Theorem 3.6

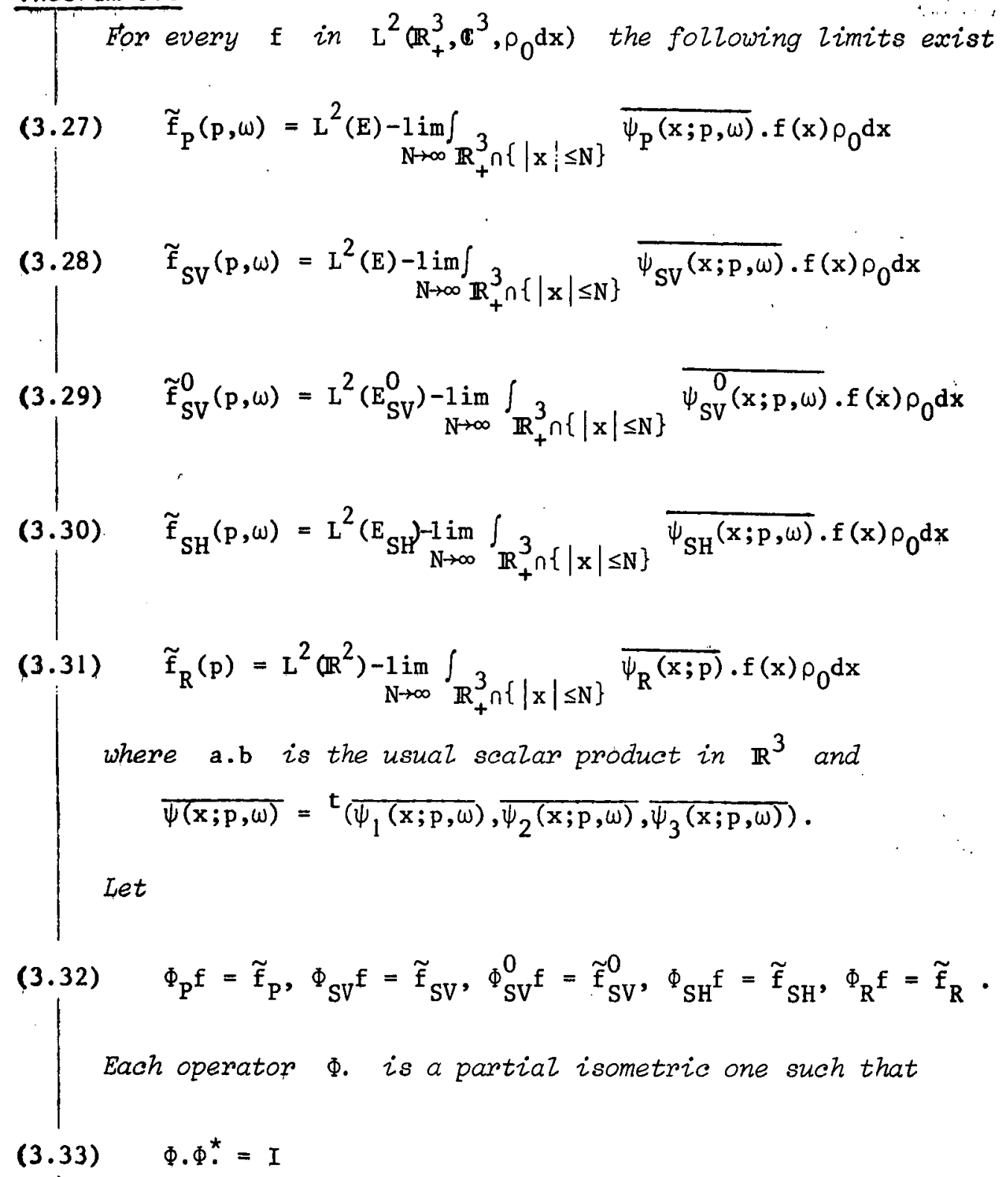

Moreover the following limits exist in $L^{2}\left(\mathbb{R}_{+}^{3}, \mathbb{C}^{3}, \rho_{0} \mathrm{dx}\right)$ :

(3.34) $\left(\Phi_{\mathrm{P}}^{*} \tilde{\mathrm{f}}_{\mathrm{P}}\right)(\mathrm{x})=\mathrm{L} \cdot \mathrm{i} \cdot \mathrm{m} \cdot \int_{\mathrm{E}} \psi_{\mathrm{P}}(\mathrm{x} ; \mathrm{p}, \omega) \tilde{\mathrm{f}}_{\mathrm{P}}(\mathrm{p}, \omega) \mathrm{dpd} \omega$

$(3.35) \quad\left(\Phi_{S V}^{\star} \tilde{\mathrm{f}}_{S V}\right)(x)=\mathrm{L} \cdot \dot{i} \cdot \mathrm{m} \cdot \int_{E} \psi_{S V}(\mathrm{x} ; \mathrm{p}, \omega) \tilde{\mathrm{f}}_{S V}(\mathrm{p}, \omega) \mathrm{dpd} \omega$ 


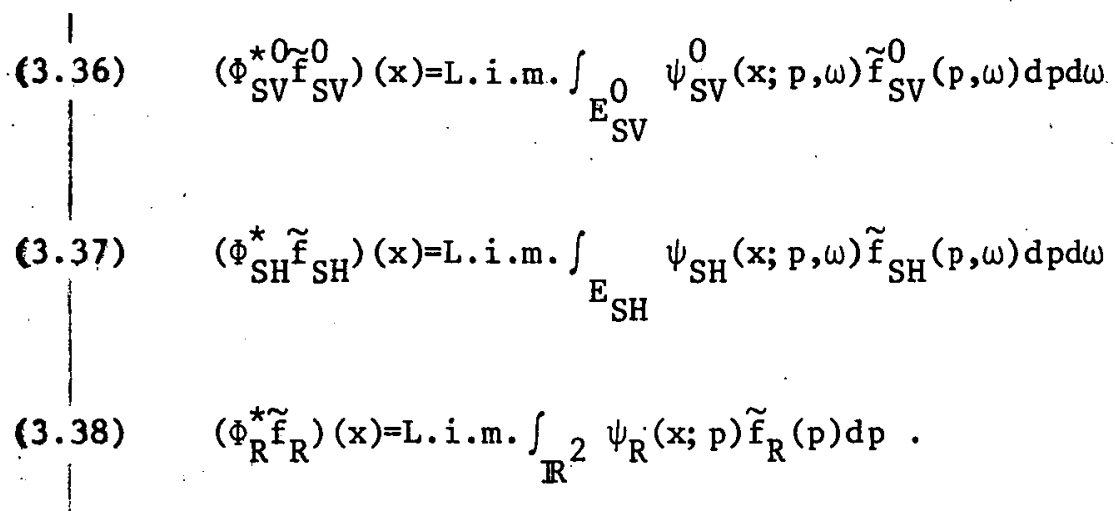

Let $\Phi$ be the following application

$$
\Phi f=\left(\Phi_{P} f, \Phi_{S V} f, \Phi_{S V}^{0} f, \Phi_{S H} f, \Phi_{R} f\right)
$$

$\Phi$ is an unitary operator from $\mathrm{L}^{2}\left(\mathbb{R}_{+}^{3}, \mathbb{C}^{3}, \rho_{0} \mathrm{dx}\right)$ into

(3.40) $L^{2}(E) \oplus L^{2}(E) \oplus L^{2}\left(E_{S V}^{0}\right) \oplus L^{2}\left(E_{S H}\right) \oplus L^{2}\left(\mathbb{R}^{2}\right)$

Furthermore $\mathrm{f}$ belongs to $\mathrm{D}\left(\mathrm{A}_{0}\right)$ if and only if

(3.41)

$$
\begin{aligned}
& \int_{E} \omega^{4}\left(\left|\tilde{\mathrm{f}}_{\mathrm{P}}(\mathrm{p}, \omega)\right|^{2}+\left|\tilde{\mathrm{f}}_{S V}(p, \omega)\right|^{2}\right) \mathrm{dpd} \omega+\int_{E_{S V}^{0}} \omega^{4}\left|\tilde{\mathrm{f}}_{S V}^{0}(p, \omega)\right|^{2} \mathrm{dpd} \omega \\
& +\int_{E_{S H}} \omega^{4}\left|\tilde{\mathrm{f}}_{S H}(p, \omega)\right|^{2} \mathrm{dpd} \omega+\int_{\mathbb{R}^{2}}|p|^{4}\left|\tilde{\mathrm{f}}_{\mathrm{R}}(\mathrm{p})\right|^{2} \mathrm{dp}<\infty
\end{aligned}
$$

and

$$
\Phi\left(A_{0} f\right)=\left(\omega^{2} \widetilde{\mathrm{f}}_{P}, \omega^{2} \widetilde{\mathrm{f}}_{S V}, \omega^{2} \widetilde{\mathrm{f}}_{\mathrm{SV}}^{0}, \omega^{2} \widetilde{\mathrm{f}}_{\mathrm{SH}}, \mathrm{c}_{\mathrm{R}}^{2}|\mathrm{P}|^{2} \widetilde{\mathrm{f}}_{\mathrm{R}}\right)
$$

if $f \in D\left(A_{0}\right)$.

In particular $\mathrm{A}_{0}$ is an absolutely continuous selfadjoint operator whose spectrum is $[0, \infty)$.

The limiting absorption principle for $A_{0}$ is a consequence of Theorem 3.6. The proof will be exactly the same as for the stratified media (cf. [6], [29]). Thus we only give the main results for $A_{0}$. 
Let $s_{1}$ and $s_{2}$ be two real numbers. We denote $L^{2 ; s_{1}, s_{2}}\left(\mathbb{R}_{+}^{3}, c^{3}\right)$ the space of all measurable $\mathfrak{c}^{3}$ valued functions on $\mathbb{R}_{+}^{3}$ defined by

$$
\mathrm{L}^{2 ; s_{1}, s_{2}}\left(\mathbb{R}_{+}^{3}, \mathbb{C}^{3}\right)=\left\{u(x):\left(1+x_{1}^{2}+x_{2}^{2}\right)^{s_{1} / 2}\left(1+x_{3}^{2}\right)^{s_{2} / 2} u(x) \in L^{2}\left(\mathbb{R}_{+}^{3}, \mathbb{C}^{3}\right)\right\} .
$$

In $L^{2 ; s_{1}, s_{2}}\left(\mathbb{R}_{+}^{3}, \mathbb{C}^{3}\right)$ we introduce the norm

(3.44) $\quad\|\mathrm{u}\|_{0 ; s_{1}, s_{2}}^{2}=\int_{\mathbb{R}_{+}^{3}}\left(1+x_{1}^{2}+x_{2}^{2}\right)^{s_{1}}\left(1+x_{3}^{2}\right)^{s_{2}} u(x) \cdot \overline{u(x)} d x$.

More generally we consider weighted Sobolev $L^{2}$ spaces $\mathrm{H}^{\mathrm{m} ; \mathrm{s}_{1}, \mathrm{~s}_{2}}\left(\mathbb{R}_{+}^{3}, \mathrm{C}^{3}\right)$ defined for any integer $m \geq 0$ by

(3.45) $\quad \mathrm{H}^{\mathrm{m} ; \mathrm{s}_{1}, s_{2}}\left(\mathbb{R}_{+}^{3}, \mathbb{C}^{3}\right)=\left\{u(\mathrm{x}) ; D^{\alpha} u \in L^{2 ; s_{1}, s_{2}}\left(\mathbb{R}_{+}^{3}, \mathbb{C}^{3}\right), 0 \leq|\alpha| \leq \mathrm{m}\right\}$

$H^{m ; s_{1}, s_{2}}\left(R_{+}^{3}, \mathbb{C}^{3}\right)$ is a Hilbert space under the norm :

(3.46) $\quad\left\|\left.u\right|_{m ; s_{1}, s_{2}} ^{2}=\sum_{|\alpha| \leq m}|| D^{\alpha} u\right\|_{0 ; s_{1}, s_{2}}^{2}$.

We then have the following theorem :

Theorem 3.7 (Limiting absorption principle for $A_{0}$ )

Suppose $s_{1}>\frac{1}{2}$ and $s_{2}>\frac{1}{2} ; z \rightarrow R_{0}(z)$ is an analytic operator

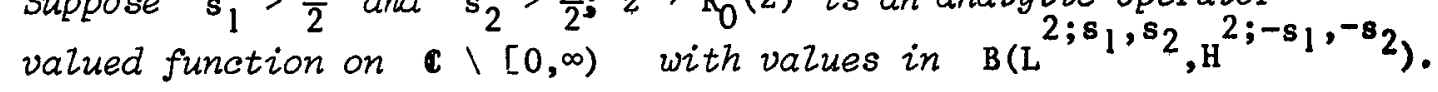

Then

(i) For any $\omega_{0}>0$ the following two limits exist in the uniform operator topology of $\mathrm{B}\left(\mathrm{L}{ }^{2 ; \mathrm{s}_{1}, \mathrm{~s}_{2}, \mathrm{H}} 2 ;-\mathrm{s}_{1},-\mathrm{s}_{2}\right)$ :

$(3.47) \quad R_{0}^{ \pm}\left(\omega_{0}^{2}\right)=\lim \lim \rightarrow \omega_{0}(z)$

Moreover, for any $\mathrm{f} \in \mathrm{L}^{2 ; \mathrm{s}_{1}, \mathrm{~s}_{2}}\left(\mathbb{R}_{+}^{3}, \mathbb{C}^{3}\right)$ the function $\mathrm{u}=\mathrm{R}_{0}^{ \pm}\left(\omega_{0}^{2}\right) \mathbf{f}$ verifies the differential equation 


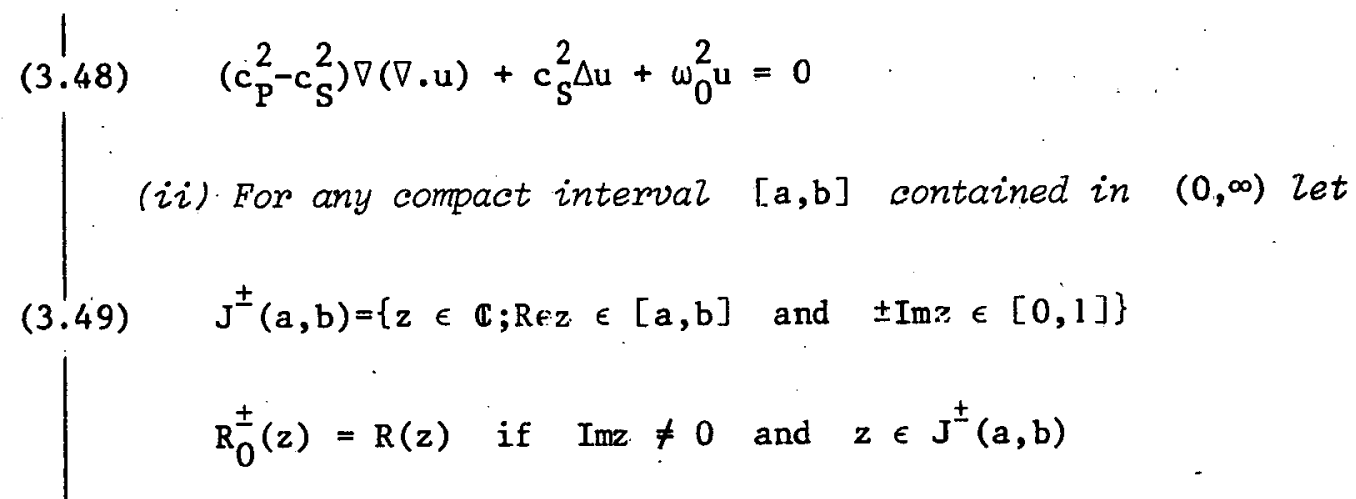

For every $\delta$ such that $\delta<\inf \left(1, s_{1}-1 / 2, s_{2}-1 / 2\right)$ there exists a positive constant $\mathrm{C}$ depending on $\mathrm{A}_{0}, \mathrm{a}, \mathrm{b}$ and $\delta$ such that

$$
|| \mathrm{R}_{0}^{+}\left(\mathrm{z}_{1}\right)-\mathrm{R}_{0}^{+}\left(\mathrm{z}_{2}\right) \|_{\mathrm{B}(\mathrm{L}} 2 ; \mathrm{s}_{1}, \mathrm{~s}_{2}, \mathrm{H}{ }^{\left.2 ;-\mathrm{s}_{1},-\mathrm{s}_{2}\right)} \leq \mathrm{C}\left|\mathrm{z}_{1}-\mathrm{z}_{2}\right|^{\delta}
$$

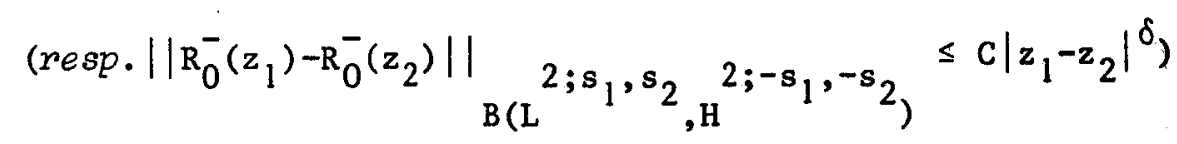

for every $\left(z_{1}, z_{2}\right) \in J^{+}(a, b) \times J^{+}(a, b) \quad\left(\right.$ resp. $\left.J^{-}(a, b) \times J^{-}(a, b)\right)$.

As in [6] in order to prove the limiting absorption principle we have to define generalized trace operators associated with $\mathrm{A}_{0}$. For any $\omega>0$ let

$$
\begin{aligned}
& E(\omega)=\left\{p \in \mathbb{R}^{2} ;(p, \omega) \in E\right\} \\
& E_{S V}^{0}(\omega)=\left\{p \in \mathbb{R}^{2} ;(p, \omega) \in E_{S V}^{0}\right\} \\
& E_{S H}(\omega)=\left\{p \in \mathbb{R}^{2} ;(p, \omega) \in E_{S H}\right\} \\
& E_{R}(\omega)=\left\{p \in \mathbb{R}^{2} ; c_{R}|p|=\omega\right\} .
\end{aligned}
$$

We then have 


\section{Proposition 3.8}

Suppose $s_{1}>1 / 2$ and $s_{2}>1 / 2$. For any $\omega>0$ there exist

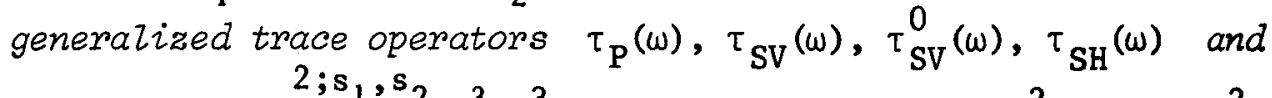
$\tau_{R}(\omega)$ from $L^{2 ; s_{1}, s_{2}}\left(\mathbb{R}_{+}^{3}, \mathbb{C}^{3}\right)$ into, respectively, $L^{2}(E(\omega)), L^{2}(E(\omega))$, $\mathrm{L}^{2}\left(\mathrm{E}_{\mathrm{SV}}^{0}(\omega)\right), \mathrm{L}^{2}\left(\mathrm{E}_{\mathrm{SH}}(\omega)\right)$, and $\mathrm{L}^{2}\left(\mathrm{E}_{\mathrm{R}}(\omega)\right)$, such that for any $f \in C_{0}^{\infty}\left(\mathbb{R}_{+}^{3}, \mathbb{c}^{3}\right):$

$$
\begin{aligned}
& \left(\tau_{P}(\omega) f\right)(p)=\tilde{f}_{P}(p, \omega) \\
& \left(\tau_{S V}(\omega) f\right)(p)=\tilde{f}_{S V}(p, \omega) \\
& \left(\tau_{S V}^{0}(\omega) f\right)(p)=\tilde{f}_{S V}^{0}(p, \omega) \\
& \left(\tau_{S H}(\omega) f\right)(p)=\tilde{f}_{S H}(p, \omega) \\
& \left(\tau_{R}(\omega) f\right)(p)=\tilde{f}_{R}(p) .
\end{aligned}
$$

Furthermore for any $f \in L^{2}\left(\mathbb{R}_{+}^{3}, \mathbb{C}^{3}\right)$ one has

$$
\left\|\left.\tau_{P}(\omega) f\right|_{L^{2}(E(\omega))} \leq M(\omega)\right\| f \|_{0 ; s_{1}, s_{2}}
$$

$$
\text { (resp. } \left.\left\|\tau_{S V}(\omega) f\right\|,\left\|\tau_{S V}^{0}(\omega) f\right\|,\left\|\tau_{S H}(\omega) f\right\|,\left\|\tau_{R}(\omega) f\right\|\right) \text {. }
$$

where $M(\omega)$ is a continuous function on $(0, \infty)$.

The proof of proposition 3.8 is exactly as in [6].

Now, for $f$ and $g$ in $C_{0}^{\infty}\left(\mathbb{R}_{+}^{3}\right)$, we have

$$
\begin{aligned}
\int_{\mathbb{R}_{+}^{3}}{ }^{R}(z) f \cdot g \rho_{0} d x= & B_{P}(z, f, g)+B_{S V}(z, f, g)+B_{S V}^{0}(z, f, g)+ \\
& +B_{S H}(z, f, g)+B_{R}(z, f, g)
\end{aligned}
$$

where 


$$
\begin{aligned}
& B_{P}(z, f, g)=\int_{0}^{\infty} h_{P}(\omega, f, g) \frac{d \omega}{\omega^{2}-z} \\
& \text { (resp. } \left.B_{S V}(z, f, g), \ldots\right)
\end{aligned}
$$

with

$$
\begin{aligned}
& h_{P}(\omega, f, g)=\int_{E(\omega)} \widetilde{f}_{P}(p, \omega) \overline{\tilde{g}_{P}(p, \omega)} d \sigma_{P}(\omega) \\
& \text { (resp. } \left.\quad h_{S V}(\omega, f, g), \ldots\right)
\end{aligned}
$$

where $d \sigma_{P}(\cdot)$ is the measure on $E(\omega)$ induced by the Lebesgue one.
Proposition 3.8 gives a meaning to (3.56) for any $f$ and $g$ in $L^{2 ; s_{1}, s_{2}}\left(\mathbb{R}_{+}^{3}, \mathbb{C}^{3}\right)$.

Useful properties of $h_{P}(., .,$.$) are now described in the following$ lemma.

\section{Lemma 3.9}

Suppose $s_{1}>1 / 2, s_{2}>1 / 2, \delta<\inf \left(s_{1}-1 / 2, s_{2}-1 / 2\right)$ and $\delta \in[0,1]$ Then there exist two continuous positive functions $M($.$) and M(.,$. respectively, on $(0, \infty)$ and $(0, \infty) \times(0, \infty)$ such that

$i^{(3.59)}\left|h_{P}(\omega, f, g)-h_{P}\left(\omega^{\prime}, f, g\right)\right| \leq M\left(\omega, \omega^{\prime}\right)\left|\omega-\omega^{\prime}\right|^{\delta}|| f||_{0 ; s_{1}, s_{2}}|| g \|_{0 ; s_{1}, s_{2}}$ (resp. $\left.\left.\left|\mathrm{h}_{\mathrm{SV}}(\omega, \mathrm{f}, \mathrm{g})-\mathrm{h}_{\mathrm{SV}}\left(\omega^{\prime}, \mathrm{f}, \mathrm{g}\right)\right|, \ldots\right)\right)$. .

It follows from the lemma 3.9 that $B_{P}\left(\omega_{0}^{2} \pm i \varepsilon, f, g\right)$ (resp. $B_{S V}\left(\omega_{0}^{2} \pm i \varepsilon, f, g\right), \ldots$ ) tends to a limit denoted by $B_{P}\left(\omega_{0}^{2} \pm i 0, f, g\right)$ when $\varepsilon$ tends to $0_{+} \cdot$ We have 
(3.60) $\quad B_{P}\left(\omega_{0}^{2} \pm i 0, f, g\right)=P V \int_{E} \frac{\widetilde{\mathrm{f}}_{P}(p, \omega) \overline{\tilde{g}_{P}(p, \omega)}}{\omega^{2}-\omega_{0}^{2}} d p d \omega \pm i \frac{\pi}{2 \omega_{0}} h_{P}\left(\omega_{0}, f, g\right)$ (resp. $\left.\quad B_{S V}\left(\omega_{0}^{2} \pm i o, f, g\right), \ldots\right)$.

From the above results and from the proof of theorem 4.1 of [3] we immediately deduce (i) of theorem 3.7. Then ( $i i)$ is a consequence of (3.56), (3.57) and (3.59). Thus theorem 3.7 is proved.

Finally we conclude this section with three remarks.

\section{$\underline{\text { Remark } 1}$}

As for the remarks 2.7 and 2.8 in [6] we have the following lemma

\section{Lemma 3.10}

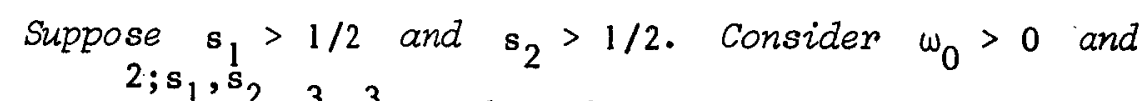
$\mathrm{f} \in \mathrm{L}^{2 ; \mathrm{s}_{1}, \mathrm{~s}_{2}}\left(\mathbb{R}_{+}^{3}, \mathbb{c}^{3}\right)$. Then the following statements are equivalent :

$(3.61)(i) \quad \mathrm{R}_{0}^{+}\left(\omega_{0}^{2}\right) \mathrm{f}=\mathrm{R}_{0}^{-}\left(\omega_{0}^{2}\right) \mathrm{f}$

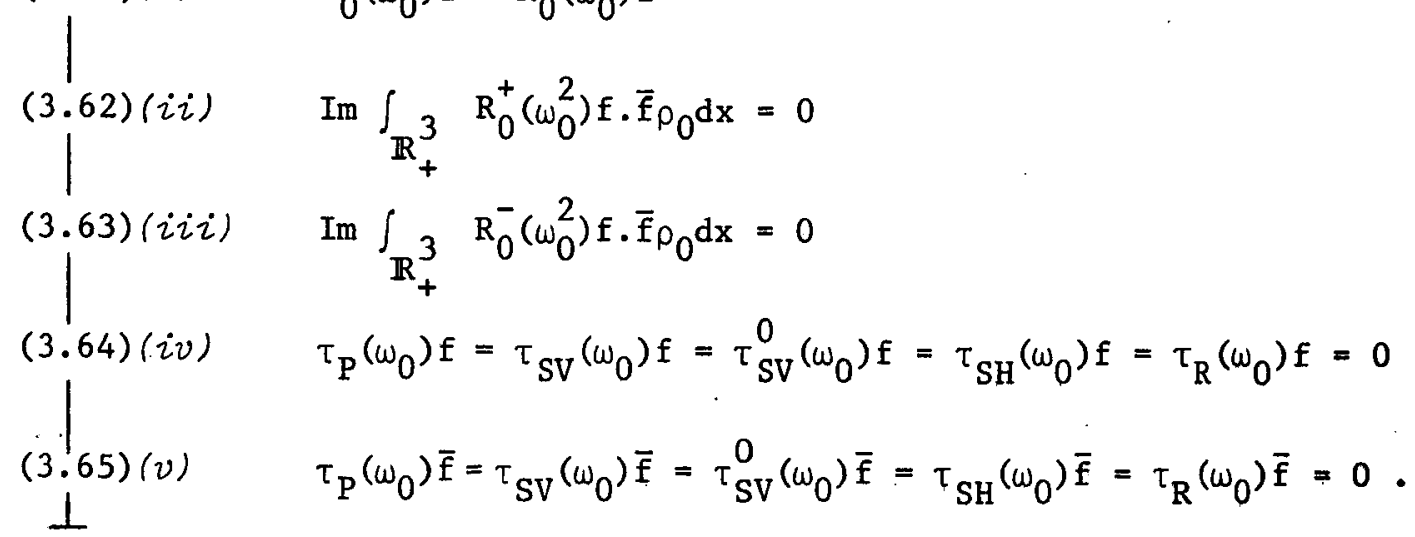

Remark 2

Consider the following Cauchy problem :

(3.66) $\quad \frac{d^{2} u}{d t^{2}}+A_{0} u=0$ 
(3.67). $u(0)=f \quad \frac{d u}{d t}(0)=g$

with $f \in D\left(A_{0}^{1 / 2}\right)$ and ${ }^{\circ} g \in L^{2}\left(\mathbb{R}_{+}^{3}, \mathbb{C}^{3}, \rho_{0} d x\right)$.

Theorem 3.6 implies the following representation of the solution $u(t)$ of $(3.66)$ and $(3.67)$

$$
u(t, x)=L \cdot i \cdot m \cdot \int_{E} \psi_{P}(x ; p, \omega)\left[\cos \omega t \tilde{f}_{P}(p, \omega)+\frac{\sin \omega t}{\omega} \tilde{g}_{P}(p, \omega)\right] d p d \omega
$$

$+\mathrm{L} \cdot \mathrm{i} \cdot \mathrm{m} \cdot \int_{\mathrm{E}} \psi_{S V}(\mathrm{x} ; \mathrm{p}, \omega)\left[\cos \omega t \widetilde{\mathbf{f}}_{S V}(p, \omega)+\frac{\sin \omega t}{\omega} \tilde{\mathrm{g}}_{S V}(p, \omega)\right] \mathrm{dpd} \omega$

$$
\begin{aligned}
& +\mathrm{L} \cdot \mathrm{i} \cdot \mathrm{m} \cdot \int_{E_{S V}^{0}}^{0} \psi_{S V}^{0}(\mathrm{x} ; \mathrm{p}, \omega)\left[\cos \omega t \tilde{\mathrm{f}}_{S V}^{0}(p, \omega)+\frac{\sin \omega t}{\omega} \tilde{g}_{S V}^{0}(p, \omega)\right] \mathrm{dpd} \omega \\
& +\mathrm{L} \cdot \mathrm{i} \cdot \mathrm{m} \cdot \int_{\mathrm{E}_{\mathrm{SH}}} \psi_{\mathrm{SH}}(\mathrm{x} ; \mathrm{p}, \omega)\left[\cos \omega t \tilde{\mathrm{f}}_{\mathrm{SH}}(\mathrm{p}, \omega)+\frac{\sin \omega t}{\omega} \tilde{\mathrm{g}}_{\mathrm{SH}}(\mathrm{p}, \omega)\right] \mathrm{dpd} \omega \\
& + \text { L.i.m. } \int_{\mathbb{R}^{2}} \psi_{R}(\mathrm{x} ; \mathrm{p})\left[\cos c_{R}|\mathrm{p}| t \widetilde{\mathrm{F}}_{\mathrm{R}}(\mathrm{p})+\frac{\sin \mathrm{c}_{\mathrm{R}}|\mathrm{p}| \mathrm{t}}{\mathrm{c}_{\mathrm{R}}|\mathrm{p}|} \tilde{\mathrm{g}}_{\mathrm{R}}(\mathrm{p})\right] \mathrm{d} \mathrm{p} \text {. }
\end{aligned}
$$

From (3.68) it is not difficult to deduce that $u(t,$.$) behaves$ when $t$ tends to $+\infty$ as a sum of an outgoing $\mathrm{P}$-wave, propagating with velocity $c_{p}$, two outgoing $s$-waves propagating with velocity. $c_{S}$ and an outgoing cylindrical Rayleigh surface wave propagating with velocity $c_{R}$. This is a consequence of the stationary phase method as in [33].

\section{Remark 3}

For the half space $\mathbb{R}_{+}^{2}$ the above results are exactly the same as for $\mathbb{R}_{+}^{3}$. The only difference is that for $n=2$ the operator $A_{0}$ has only four generalized eigenfunctions denoted by $\psi_{\mathrm{P}}(\mathrm{x}, \mathrm{p}, \omega) ; \psi_{\mathrm{S}}(\mathrm{x}, \mathrm{p}, \omega)$ $\psi_{S}^{0}(x, p, w)$ and $\psi_{R}(x, p)$ where $x=\left(x_{1}, x_{2}\right) \in \mathbb{R}_{+}^{2}$ and $p \in \mathbb{R}$. This can be proved by following the same method as for $\mathfrak{n}=3$. 
Let $\xi_{p}\left(\omega^{2}\right)=\left(\omega^{2} / c_{p}^{2}-p^{2}\right)^{1 / 2}$ if $\omega>c_{p}|p|, \xi_{p}^{\prime}\left(\omega^{2}\right)=\left(p^{2}-\omega^{2} / c_{p}^{2}\right)^{1 / 2}$ if $c_{S}|p|<\omega<c_{p}|p|$ and $\xi_{S}\left(\omega^{2}\right)=\left(\omega_{0}^{2} / c_{S}^{2}-p^{2}\right)^{1 / 2}$ if $\omega>c_{S}|p|$.

Let

$(3.69)$

$$
E=\left\{(p, \omega) \in \mathbb{R}_{+}^{2} ; \omega>c_{p}|p .|\right\}
$$

$$
\mathbf{E}_{S}^{0}=\left\{(p, \omega) \in \mathbb{R}_{+}^{2} ; c_{S}|p|<\omega<c_{P}|p|\right\}
$$

For any $(p, \omega) \in E$ we have

$$
\psi_{\mathrm{p}}(\mathrm{x}, \mathrm{p}, \omega)=\frac{1}{2 \pi} e^{i p x_{1}} \frac{1}{\left(\omega \rho_{0} \xi_{\mathrm{P}}\left(\omega^{2}\right)\right)^{1 / 2}}\left\{e^{-i \xi_{\mathrm{P}}\left(\omega^{2}\right) x_{2}} \mathrm{t}_{\left(\mathrm{p},-\xi_{\mathrm{P}}\left(\omega^{2}\right)\right)} .\right.
$$

$$
\begin{aligned}
& +\frac{4 p\left(\omega^{2} / c_{s}-2 p^{2}\right) \xi_{p}\left(\omega^{2}\right)}{\left(\omega^{2} / c^{2}-2 p^{2}\right)^{2}+4 p^{2} \xi_{p}\left(\omega^{2}\right) \xi_{S}\left(\omega^{2}\right)} e^{i \xi_{s}\left(\omega^{2}\right) x_{2}} t_{\left(\xi_{s}\left(\omega^{2}\right),-p\right)}
\end{aligned}
$$

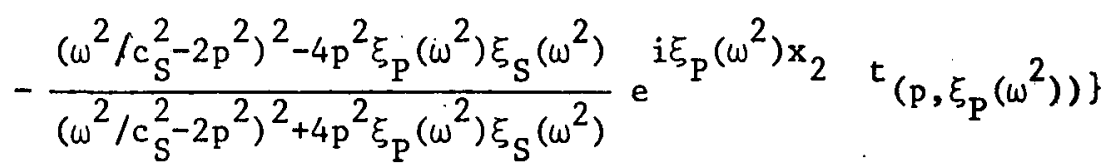

$$
\begin{aligned}
& \psi_{S}(x, p, \omega)=\frac{1}{2 \pi} e^{i p x_{1}} \frac{1}{\left(\omega \rho_{0} \xi_{S}\left(\omega^{2}\right)\right)^{1 / 2}}\left\{e^{-i \xi_{S}\left(\omega^{2}\right) x_{2}}{ }^{t}\left(\xi_{S}\left(\omega^{2}\right), p\right)\right. \\
& +\frac{\left(\omega^{2} / c^{2}-2 p^{2}\right)^{2}-4 p^{2} \xi_{P}\left(\omega^{2}\right) \xi_{S}\left(\omega^{2}\right)}{\left(\omega^{2} / c_{S}^{2}-2 p^{2}\right)^{2}+4 p^{2} \xi_{P}\left(\omega^{2}\right) \xi_{S}\left(\omega^{2}\right)} e^{i \xi_{S}(\omega) x_{2}}{ }^{t}\left(\xi_{S}\left(\omega^{2}\right),-p\right) \\
& \left.+\frac{4 p\left(\omega^{2} / c^{2} s^{\left.-2 p^{2}\right) \xi_{S}\left(\omega^{2}\right)}\right.}{\left(\omega^{2} / c^{2} s^{2} p^{2}\right)^{2}+4 p^{2} \xi_{p}\left(\omega^{2}\right) \xi_{S}\left(\omega^{2}\right)} e^{i \xi_{p}\left(\omega^{2}\right) x_{2}}{ }^{t}\left(p, \xi_{p}\left(\omega^{2}\right)\right)\right\} .
\end{aligned}
$$

For any $(p, w) \in E_{S}^{0}$ we have 
29

$$
\begin{aligned}
\psi_{S}^{0}(x, p, \omega) & =\frac{1}{2 \pi} e^{i p x_{1}} \frac{1}{\left(\omega \rho_{0} \xi_{S}\left(\omega^{2}\right)\right)}{ }^{1 / 2}\left\{e^{-i \xi_{S}\left(\omega^{2}\right) x_{2}} t_{\left(\xi_{S}\right.}\left(\omega^{2}\right), p\right) \\
& +\frac{\left(\omega^{2} / c_{S}^{2}-2 p^{2}\right)^{2}-4 i p^{2} \xi_{P}^{\prime}\left(\omega^{2}\right) \xi_{S}\left(\omega^{2}\right)}{\left(\omega^{2} / c_{S}^{2}-2 p^{2}\right)^{2}+4 i p^{2} \xi_{P}^{\prime}\left(\omega^{2}\right) \xi_{S}\left(\omega^{2}\right)} e^{i \xi_{S}\left(\omega^{2}\right) x_{2} t\left(\xi_{S}\left(\omega^{2}\right),-p\right)} \\
& \left.+\frac{4 p\left(\omega^{2} / c_{S}^{2}-2 p^{2}\right) \xi_{S}\left(\omega^{2}\right)}{\left(\omega^{2} / c_{S}^{2}-2 p^{2}\right)^{2}+4 i p^{2} \xi_{P}^{\prime}\left(\omega^{2}\right) \xi_{S}\left(\omega^{2}\right)} e^{-\xi_{P}^{\prime}\left(\omega^{2}\right) x_{2}} t\left(p, i \xi_{P}^{\prime}\left(\omega^{2}\right)\right)\right\}
\end{aligned}
$$

and for any $p \in \mathbb{R}$ we have

$$
\begin{aligned}
& \psi_{R}(x, p)=\frac{c}{2 \pi} e^{i p x_{1}}|p|^{1 / 2}\left\{\left(2-\frac{c_{R}^{2}}{\left.c_{S}^{2}\right)} e^{-\mid p\left(1-c_{R}^{2} / c_{P}^{2}\right)^{1 / 2} x_{2}}\right.\right. \\
& t_{\left(i,-\left(1-c_{R}^{2} / c_{P}^{2}\right)^{1 / 2} \frac{p}{|p|}\right)} \\
& \left.\left.-2\left(1-\frac{c_{R}^{2}}{c_{P}^{2}}\right)^{1 / 2} e^{-|p|\left(1-c_{R}^{2} / c_{S}^{2}\right)^{1 / 2} x_{2}} t_{\left(i\left(1-c_{R}^{2} / c_{S}^{2}\right) 1 / 2\right.},-\frac{p}{|p|}\right)\right\}
\end{aligned}
$$

where $C$ is a positive constant such that

(3.74) $\quad 4 \pi^{2} \int_{0}^{\infty}\left|\psi_{R}(x, p)\right|^{2} \rho_{0} d x_{2}=1$. 
4. THE DIVISION THEOREM FOR $A_{0}$

This section is devoted to the generalization for $A_{0}$ of theorem $\mathrm{B} .1$ proved by S. Agmon in [3] (see also [18], [25], [27]). This is the so called division theorem for $A_{0}$. Roughly speaking, this theorem says that if the generalized traces of $f \in L^{2 ; s_{1}, s_{2}}\left(\mathbb{R}_{+}^{3}, \mathbb{C}^{3}\right)$ vanish on $E(\omega), E(\omega)$, $E_{S V}^{0}(\omega), E_{S H}(\omega)$ and $E_{R}(\omega)$ then the function $u=R_{0}^{ \pm}\left(\omega^{2}\right) f$ has a better decay at infinity than it is expected from theorem 3.7 .

As a consequence of the division theorem we deduce the limiting absorption principle for $A$ without using any explicit radiation condition and any subsequent uniqueness theorem as the Rellich's theorem for the Laplacian in $\mathbb{R}^{n}$. Moreover the division theorem enables us to prove decay properties on the $x_{3}$-direction of the eigenfunctions for $A$ associated with the strictly positive eigenvalues.

Thus we have

Theorem 4.1 (the division theorem for $A_{0}$ )

Assume $\mathrm{s}_{1}>1 / 2$ and $\mathrm{s}_{2}>1 / 2$. Let $\mathrm{f} \in \mathrm{L}^{2 ; \mathrm{s}_{1}, \mathrm{~s}_{2}}\left(\mathbb{R}_{+}^{3}, \mathbb{C}^{3}\right)$ and let $\sigma$ be a strictly positive number such that

$$
\begin{aligned}
& \text { (4.1) } \quad \tau_{P}(\sigma) f=\tau_{S V}(\sigma) f=\tau_{S V}^{0}(\sigma) f=\tau_{S H}(\sigma) f=\tau_{R}(\sigma) f=0 . \\
& \text { Let } \tilde{s}_{1} \text { and } \tilde{s}_{2} \text { be two real numbers such that } \\
& (4.2) \quad \tilde{s}_{1}= \begin{cases}s_{1}-1 & \text { if } 1 / 2<s_{1} \leq 1 \\
0 & \text { if } 1<s_{1}\end{cases}
\end{aligned}
$$

and

$$
\tilde{s}_{2}<\inf \left(2 s_{1}-3 / 2, s_{2}-1\right) \text { if } 1 / 2<s_{2} \leq 1
$$

$$
\tilde{s}_{2}<\inf \left(2 s_{1}-3 / 2, s_{2} / 2-1 / 2,1\right) \text { if } 1<s_{2} \text {. }
$$


Then

(4.4) $R_{0}^{+}\left(\sigma^{2}\right) f=R_{0}^{-}\left(\sigma^{2}\right) f \in L^{2 ; \tilde{s}_{1}, \tilde{s}_{2}}\left(\mathbb{R}_{+}^{3}, \mathbb{C}^{3}\right)$

and

$(4.5)$

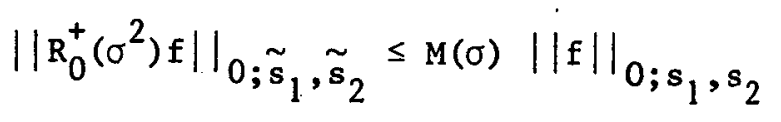

where $\mathrm{M}($.$) is a positive continuous function on (0, \infty)$ depending only on $s_{1}, s_{2}$ and $\tilde{s}_{2}$.

\section{Remark}

Continuity of $M($.$) is useful in proving that the positive eigenva-$ lues of $A$ can accumulate only at 0 and $+\infty$.

The proof of theorem 4.1 is very long and depends on several propositions.

We have (cf. section 3):

$$
\begin{aligned}
& A_{0}=\mathscr{\not}^{-1} \hat{A}_{0} \oiint=\mathscr{Q}^{-1}\left(\int_{p \in \mathbb{R}^{2}}^{\oplus} U\left(p_{1}, p_{2}\right) \Pi\left(B_{1}(|p|) \oplus B_{2}(|p|)\right) \Pi U\left(p_{1},-p_{2}\right) d p\right) \mathscr{\not} \\
& \text { For any } z \in \mathbb{C} \backslash[0, \infty) \text { let } \\
& R_{1}(z)=\left(B_{1}(|p|)-z I\right)^{-1} \text { and } R_{2}(z)=\left(B_{2}(|p|)-z I\right)^{-1} \text {. }
\end{aligned}
$$

We then have

$$
\begin{aligned}
& \text { (4.7) } \quad R_{0}(z)=\mathscr{\bigotimes}^{-1}\left(\int_{p \in \mathbb{R}^{2}}^{\oplus} U\left(p_{1}, p_{2}\right) \Pi\left(R_{1}(z) \oplus R_{2}(z)\right) \Pi U\left(p_{1},-p_{2}\right) d p\right) \& \\
& \text { Assume } \mathbf{s}_{1}>1 / 2, \mathbf{s}_{2}>1 / 2 \text { and } \mathbf{f} \in \mathrm{L}^{2}\left(\mathbb{R}_{+}^{3}, \mathbb{C}^{3}\right) \text {. Let } \\
& \text { (4.8) } \quad g\left(p, x_{3}\right)={ }^{t}\left(g_{1}\left(p, x_{3}\right), g_{2}\left(p, x_{3}\right), g_{3}\left(p, x_{3}\right)\right)=U\left(p_{1},-p_{2}\right)\left(\&_{f}\right)\left(p, x_{3}\right)
\end{aligned}
$$


and

$$
g^{\prime}\left(p, x_{3}\right)=\left(g_{1}\left(p, x_{3}\right), g_{3}\left(p, x_{3}\right)\right) \text {. }
$$

Thus $g \in \mathrm{L}^{2 ; 0, \mathrm{~s}_{2}}\left(\mathbb{R}_{+}^{3}, \mathrm{C}^{3}, \mathrm{dpdx_{3 }}\right)$.

The method used in section 3 gives a meaning to $R_{1}^{ \pm}\left(\sigma^{2}\right) g^{\prime}$ and to $\mathrm{R}_{2}^{ \pm}\left(\sigma^{2}\right) g_{2}$. Thus we have

(4.10) $\quad\left(\mathscr{W}_{0}^{ \pm}\left(\sigma^{2}\right) f\right)\left(p, x_{3}\right)=U\left(p_{1}, p_{2}\right) \Pi\left(R_{1}^{ \pm}\left(\sigma^{2}\right) g^{\prime}(p,.) \oplus R_{2}^{ \pm}\left(\sigma^{2}\right) g_{2}(p,).\right)\left(x_{3}\right)$.

Thus the proof of theorem 4.1 is the consequence of $(4.10)$ and of a careful study of $R_{1}^{ \pm}\left(\dot{\sigma}^{2}\right) g^{\prime}(p)$ and $R_{2}^{ \pm}\left(\sigma^{2}\right) g_{2}(p)$.

First of all we have to give definitions of several analytic functions in the cut complex plane

(i) $\xi_{S}(z)$ denotes the branch of $\left(z / c_{S}^{2}-|p|^{2}\right)^{1 / 2}$ on $\mathbb{C} \backslash\left[-\infty, c_{S}^{2}|p|^{2}\right]$ such that $\operatorname{Re} \xi_{S}(z)>0$.

$\tilde{\xi}_{S}(z)$ denotes the branch of $\left(z / c_{S}^{2}-|p|^{2}\right)^{1 / 2}$ on $\mathbb{C} \backslash\left[c_{S}^{2}|p|^{2}, \infty\right)$ such that $\operatorname{Im} \tilde{\xi}_{S}(z)>0$.

$\xi_{S}^{\prime}(z)$ denotes the branch of $\left(|p|^{2}-z / c_{S}^{2}\right)^{1 / 2}$ on $\mathbb{C} \backslash\left[c_{S}^{2}|p|^{2}, \infty\right)$ such that $\operatorname{Re} \xi_{S}^{\prime}(z)>0$.

(ii) $\xi_{p}(z)$ denotes the branch of $\left(z / c_{p}^{2}-|p|^{2}\right)^{1 / 2}$ on $\mathbb{C} \backslash\left(-\infty, c_{p}^{2}|p|^{2}\right]$ such that $\operatorname{Re} \xi_{\mathrm{P}}(z)>0$.

$\tilde{\xi}_{P}(z)$ denotes the branch of $\left(z / c_{P}^{2}-|p|^{2}\right)^{1 / 2}$ on $\mathbb{C} \backslash\left[c_{p}^{2}|p|^{2}, \infty\right)$ such that $\operatorname{Im} \tilde{\xi}_{P}(z)>0$.

$\xi_{p}^{\prime}(z)$ denotes the branch of $\left(|p|^{2}-z / c_{p}^{2}\right)^{1 / 2}$ on $\mathbb{C} \backslash\left[c_{p}^{2}|p|^{2}, \infty\right)$ such that $\operatorname{Re} \xi_{\mathrm{P}}^{\prime}(\mathrm{z})>0$.

One verifies that

(4.11) $\operatorname{Im} \xi_{S}(z) \operatorname{Im} z>0$ and $\operatorname{Im} \xi_{S}^{\prime}(z) \operatorname{Im} z<0$ if $\operatorname{Imz} \neq 0$.

Moreover one has 
(4.12) $\xi_{S}(z)=\tilde{\xi}_{S}(z)=i \xi_{S}^{\prime}(z)$ and $\xi_{P}(z)=\tilde{\xi}_{P}(z)=i \xi_{P}^{\prime}(z)$ if $\operatorname{Im} z>0$ and

(4.13) $\xi_{S}(z)=-\tilde{\xi}_{S}(z)=-i \xi_{S}^{\prime}(z)$ and $\xi_{p}(z)=-\tilde{\xi}_{P}(z)=-i \xi_{P}^{\prime}(z)$ if $\operatorname{Im} z<0$.

\subsection{Study of $R_{2}^{ \pm}\left(\sigma^{2}\right) g_{2}$}

Let

$(4.14) \quad v\left(p, x_{3}, \sigma^{2}\right)=\left(R_{2}^{+}\left(\sigma^{2}\right) g_{3}(p,).\right)\left(x_{3}\right)$

We then have

\section{Proposition 4.2}

Suppose $s_{1}>1 / 2$ and $s_{2}>1 / 2$. Let $f \in L^{2 ; s_{1}, s_{2}}\left(\mathbb{R}_{+}^{3}, \mathbb{C}^{3}\right)$ and let $\sigma$ be a strictly positive number such that

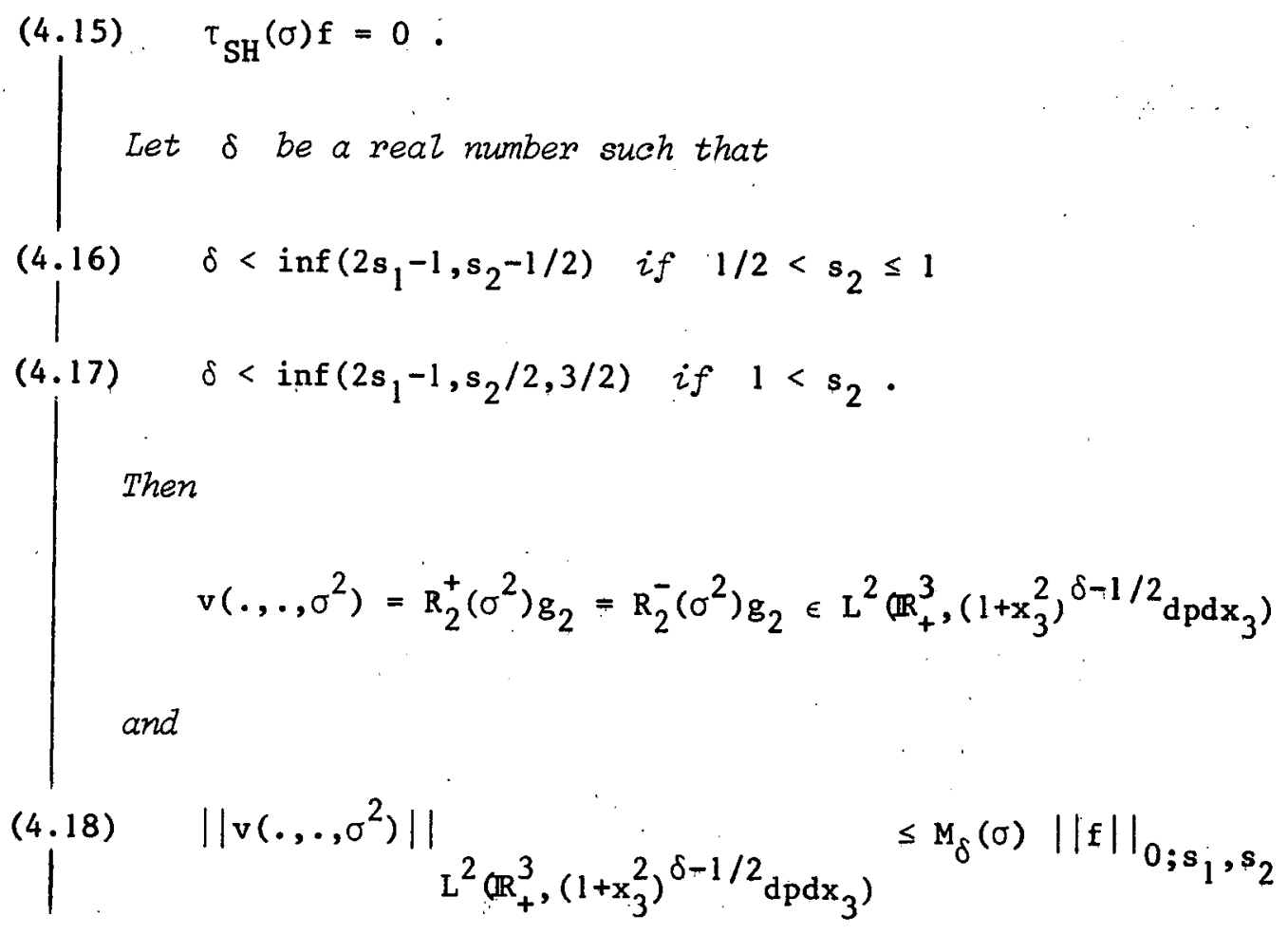


where $\mathrm{M}_{\delta}($.$) is a positive continuous function on (0, \infty)$.

(4.15) is equivalent to

(4.19) $\quad \int_{0}^{\infty} \cos \xi_{S}\left(\sigma^{2}\right) x_{3} g_{2}\left(p, x_{3}\right) d x_{3}=0$ for $|p|<\sigma / c_{s}$.

Let $x_{1}$ (resp. $\chi_{2}, \chi_{3}$ ) be the characteristic function of $\left(0, \sigma / c_{s}\right)$ (resp. $\left.\left(\sigma / c_{S}, \sqrt{2} \sigma / c_{S}\right),\left(\sqrt{2} \sigma / c_{S}, \infty\right)\right)$.

Let

$$
\begin{aligned}
& v_{i}\left(p, x_{3}, z\right)=\chi_{i}(|p|) v\left(p, x_{3}, z\right)=\chi_{i}(|p|)\left[R_{2}(z) g_{2}(p, .)\right]\left(x_{3}\right) \\
& i=1,2,3 .
\end{aligned}
$$

$v_{i}\left(p, x_{3}, z\right)$ has a meaning for $z \in \mathbb{C} \backslash[0, \infty) . v_{i}\left(p, x_{3}, \sigma^{2}\right)$ will be defined as the limit of $v_{2}\left(p, x_{3}, z\right)$ as $z$ tends to $\sigma^{2}$ such that Im $z>0$.

After having estimated each $v_{i}\left(p, x_{3}, \sigma^{2}\right)$ it will not be difficult to show as in [6] that

(4.21) $\quad v\left(p, x_{3}, \sigma^{2}\right)=\sum_{i=1}^{3} v_{i}\left(p, x_{3}, \sigma^{2}\right)$

4.1.1. Definition and estimate of $v_{1}\left(p, x_{3}, \sigma^{2}\right)$

Let

$$
\phi_{1}\left(p, x_{3}, z\right)=\cos \xi_{S}(z) x_{3} \text { and } \phi_{2}\left(p, x_{3}, z\right)=\exp i \xi_{S}(z) x_{3} .
$$

It follows from theorem XIII.3.16 in [9] that

$$
v_{1}\left(p, x_{3}, z\right)=i x_{1}(|p|)\left(c_{S}^{2} \xi_{S}(z)\right)^{-1}\left[\phi_{2}\left(p, x_{3}, z\right) \int_{0}^{x_{3}} \phi_{1}(p, y, z) g_{2}(p, y) d y\right.
$$

$$
\left.+\phi_{1}\left(p, x_{3}, z\right) \int_{x_{3}}^{\infty} \phi_{2}(p, y, z) g_{2}(p, y) d y\right] \text {. }
$$


Letting

$$
\begin{aligned}
v_{1}\left(p, x_{3}, \sigma^{2}\right)= & \lim _{\substack{z \rightarrow \sigma^{2} \\
\operatorname{Imz}>0}} v_{1}\left(p, x_{3}, z\right) \text {, we get ... } \\
v_{1}\left(p, x_{3}, \sigma^{2}\right)= & i x_{1}(|p|)\left(c_{S}^{2} \xi_{S}\left(\sigma^{2}\right)\right)^{-1}\left[e^{i \xi_{S}\left(\sigma^{2}\right) x_{3}} \int_{0}^{x_{3}} \cos \xi_{S}\left(\sigma^{2}\right) y g_{2}(p, y) d y\right. \\
& \left.+\cos \xi_{S}\left(\sigma^{2}\right) x_{3} \int_{x_{3}}^{\infty} e^{i \xi_{S}\left(\sigma^{2}\right) y} g_{2}(p, y) d y\right] .
\end{aligned}
$$

From (4.19) we deduce that

$$
\begin{aligned}
v_{1}\left(p, x_{3}, \sigma^{2}\right)=i x_{1}(|p|)\left(c_{S}^{2} \xi_{S}\left(\sigma^{2}\right)\right)^{-1} \int_{x_{3}}^{\infty} & {\left[\cos \xi_{S}\left(\sigma^{2}\right) x_{3} e^{i \xi_{s}\left(\sigma^{2}\right) y}\right.} \\
& \left.-\cos \xi_{S}\left(\sigma^{2}\right) y e^{i \xi_{S}\left(\sigma^{2}\right) x_{3}}\right] g_{2}(p, y) d y
\end{aligned}
$$

We have

(4.25) $\quad\left|e^{\text {iax }_{3}}-e^{\text {iay }}\right| \leq 2^{\gamma}|a|^{\gamma}\left|x_{3}-y\right|^{\gamma}$ for $0 \leq \gamma \leq 1$.

Thus (4.24) and (4.25) imply

(4.26) $\quad\left|v_{1}\left(p, x_{3}, \sigma^{2}\right)\right| \leq c_{X_{1}}(|p|)\left(\xi_{s}\left(\sigma^{2}\right)\right)^{\gamma-1} \int_{x_{3}}^{\infty}\left|x_{3}-y\right|^{\gamma}\left|g_{2}(p, y)\right| d y$.

For $\alpha$ such that $0 \leq \alpha<s_{2}-\gamma-1 / 2$ we have

(4.27) $\int_{x_{3}}^{\infty}\left(1+y^{2}\right)^{\gamma-s} 2 d y \leq c\left(1+x_{3}^{2}\right)^{-\alpha}$.

Therefore

$$
\left|v_{1}\left(p, x_{3}, \sigma^{2}\right)\right|^{2} \leq\left.\left. c x_{1}(|p|)\left(\xi_{S}\left(\sigma^{2}\right)\right)^{2 \gamma-2}\left(1+x_{3}^{2}\right)^{-\alpha} \int_{0}^{\infty}\left(1+y^{2}\right)^{s}\right|_{g_{2}}(p, y)\right|^{2} d y
$$

Let $(|p|, \theta)$ be the polar coordinates of $p$. The first remark to be made is that $f_{|\mathrm{p}|=r}\left|g_{2}(\mathrm{p}, \mathrm{y})\right|^{2}|\mathrm{p}| \mathrm{d} \theta$ has a meaning because. $g_{2}(., y)$ is in $\mathbf{H}^{s_{1}}\left(\mathbb{R}^{2}\right)$ for almost every " $y>0$. 
The second remark to be made is that the function $|p| \rightarrow\left(\xi_{s}\left(\sigma^{2}\right)\right)^{-\beta}$ is integrable on $\left[0, \sigma / c_{S}\right)$ for $\beta<2$.

From $(4.26)$ and these two remarks we deduce that $v_{1}\left(., ., \sigma^{2}\right)$ verifies $(4.18)$.

\section{Remark}

Just note that in the following the Young's inequality, the Peetre's inequality and this estimate:

$$
\int_{0}^{\infty} e^{-a y}(1+y) s d y \leq \Gamma(1+s) e^{a} a^{-(1+s)}, a>0,1+s>0
$$

will be of ten used without mentioning them explicitely.

\subsubsection{Definition and estimation of $v_{2}\left(p, x_{3}, \sigma^{2}\right)$}

Let

$$
\phi_{1}\left(p, x_{3}, z\right)=c h \xi_{S}^{\prime}(z) x_{3} \text { and } \phi_{2}\left(p, x_{3}, z\right)=\exp \left(-\xi_{S}^{\prime}(z) x_{3}\right)
$$

We have

$$
v_{2}\left(p, x_{3}, z\right)=x_{2}(|p|)\left(c_{S}^{2} \xi_{S}^{\prime}(z)\right)^{-1}\left[\phi_{2}\left(p, x_{3}, z\right) \int_{0}^{x_{3}} \phi_{1}(p, y, z) g_{2}(p, y) d y\right.
$$

$$
\left.+\phi_{1}\left(p, x_{3}, z\right) \int_{x_{3}}^{\infty} \phi_{2}(p, y, z) g_{2}(p, y) d y\right]
$$

and as in 4.1 .1 we get

$$
\begin{aligned}
v_{2}\left(p, x_{3}, \sigma^{2}\right) & =x_{2}(|p|)\left(c_{S}^{2} \xi_{S}^{\prime}\left(\sigma^{2}\right)\right)^{-1}\left[e^{-\xi_{S}^{\prime}\left(\sigma^{2}\right) x_{3}} \int_{0}^{x_{3}} \operatorname{ch} \xi_{S}^{\prime}\left(\sigma^{2}\right) y g_{2}(p, y) d y\right. \\
& \left.+\operatorname{ch~} \xi_{S}^{\prime}\left(\sigma^{2}\right) x_{3} \int_{x_{3}}^{\infty} e^{-\xi_{S}^{\prime}\left(\sigma^{2}\right) y} g_{2}(p, y) d y\right] .
\end{aligned}
$$

But in order to estimate (4.31) we cannot use (4.19) as for $v_{1}\left(., \ldots, \sigma^{2}\right)$ because we have $|\mathrm{p}|>\sigma / \mathrm{c}_{\mathrm{S}}$ in the case of $\mathrm{v}_{2}\left(,, \ldots, \sigma^{2}\right)$. Instead we 
adapt to our case the method followed in the proof of theorem 3.5 of [6].

Let

(4.32) $\quad p^{\prime}=p|p|^{-1}\left(2 \sigma^{2} c_{s}^{2}-|p|^{2}\right)^{1 / 2}=p|p|^{-1}\left(\sigma^{2} c_{s}^{-2}-\left(\xi_{S}^{\prime}\left(\sigma^{2}\right)\right)^{2}\right)^{1 / 2}$

and in order to simplify let

$$
\xi_{S}\left(\sigma^{2}\right)=\left(\sigma^{2} c_{S}^{-2}-\left|p^{\prime}\right|^{2}\right)^{1 / 2} \text { and } \xi_{S}^{\prime}\left(\sigma^{2}\right)=\left(|p|^{2}-\sigma^{2} c_{S}^{-2}\right)^{1 / 2} \text {. }
$$

One verifies that $\left|\mathrm{p}^{\prime}\right|<\sigma / \mathrm{c}_{S}$ and $\xi_{\mathrm{S}}\left(\sigma^{2}\right)=\xi_{S}^{\prime}\left(\sigma^{2}\right)$.

From (4.19) and (4.31) we obtain

$$
v_{2}\left(p, x_{3}, \sigma^{2}\right)=\sum_{j=1}^{4} v_{2 j}\left(p, x_{3}, \sigma^{2}\right)
$$

with

$$
v_{2 j}\left(p, x_{3}, \sigma^{2}\right)=x_{2}(|p|)\left(c_{s}^{2} \xi_{s}^{\prime}\left(\sigma^{2}\right)\right)^{-1} v_{2 j}^{\prime}\left(p, x_{3}, \sigma^{2}\right)
$$

and

$$
\begin{aligned}
& v_{21}^{\prime}\left(p, x_{3}^{\prime}, \sigma^{2}\right)=e^{-\xi_{S}^{\prime}\left(\sigma^{2}\right) x_{3}} \int_{0}^{x_{3}}\left[\operatorname{ch} \xi_{S}^{\prime}\left(\sigma^{2}\right) y-\cos \xi_{S}\left(\sigma^{2}\right) y\right] g_{2}(p, y) d y, \\
& v_{22}^{\prime}\left(p, x_{3}, \sigma^{2}\right)=e^{-\xi_{S}^{\prime}\left(\sigma^{2}\right) x_{3}} \int_{0}^{x_{3}} \cos \xi_{S}\left(\sigma^{2}\right) y\left[g_{2}(p, y)-g_{2}\left(p^{\prime}, y\right)\right] d y,
\end{aligned}
$$

$$
\begin{aligned}
& v_{23}^{\prime}\left(p, x_{3}, \sigma^{2}\right)=\operatorname{ch} \xi_{S}^{\prime}\left(\sigma^{2}\right) x_{3} \int_{x_{3}}^{\infty} e^{-\xi_{S}^{\prime}\left(\sigma^{2}\right) y}\left[g_{2}(p, y)-g_{2}\left(p^{\prime}, y\right)\right] d y, \\
& v_{24}^{\prime}\left(p, x_{3}, \sigma^{2}\right)=\int_{x_{3}}^{\infty}\left[\operatorname{ch} \xi_{S}^{\prime}\left(\sigma^{2}\right) x_{3} e^{-\xi_{S}^{\prime}\left(\sigma^{2}\right) y}-e^{-\xi_{S}^{\prime}\left(\sigma^{2}\right) x_{3}} \cos \xi_{S}\left(\sigma^{2}\right) y\right] \\
& g_{2}\left(p^{\prime}, y\right) d y .
\end{aligned}
$$

But we have the following inequalities 


$$
\left|\operatorname{ch} \xi_{S}^{\prime}\left(\sigma^{2}\right) y-\cos \xi_{S}\left(\sigma^{2}\right) y\right| \leq M_{\gamma}\left(\xi_{S}^{\prime}\left(\sigma^{2}\right)\right)^{2 \gamma}\left(1+y^{2}\right)^{\gamma} e^{\xi_{S}^{\prime}\left(\sigma^{2}\right) y}, 0 \leq \gamma \leq 1
$$

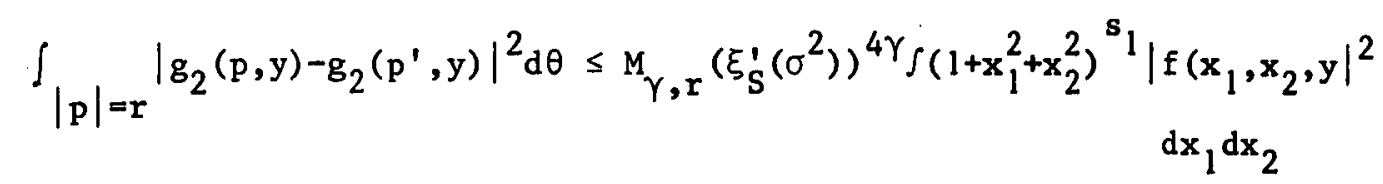

if $0 \leq \gamma \leq 1$ and $\gamma<s_{1}^{-1 / 2}$,

$$
\left|\operatorname{ch} \xi_{S}^{\prime}\left(\sigma^{2}\right) x_{3} e^{-\xi_{S}^{\prime}\left(\sigma^{2}\right) y}-e^{-\xi_{S}^{\prime}\left(\sigma^{2}\right) x_{3}} \cos \xi_{S}\left(\sigma^{2}\right) y\right| \leq M_{\gamma}\left(\xi_{S}^{\prime}\left(\sigma^{2}\right)\right)^{\gamma}\left(1+y^{2}\right)^{\gamma / 2}
$$

if $0 \leq \gamma \leq 1$ and $0 \leq x_{3} \leq y$.

From (4.35) - (4.37) we easily deduce that each $v_{2 j}$ verifies (4.18) with, respectively,

$$
\begin{aligned}
& \delta<s_{2}-1 / 2 \text { if } 1 / 2<s_{2} \leq 1 \text { for } v_{21} \\
& \delta<\inf \left(s_{2} / 2,3 / 2\right) \text { if } 1<s_{2} \text { for } v_{21} \\
& \delta<\inf \left(2 s_{1}-1,2\right) \text { for } v_{22} \\
& \delta<s_{2}-1 / 2 \text { for } v_{23} \text { and } v_{24^{\circ}} .
\end{aligned}
$$

Thus $\mathrm{v}_{2}\left(., ., \sigma^{2}\right)$ verifies $(4.18)$.

4.1.3. Definition and estimation of $v_{3}\left(p, x_{3}, \sigma^{2}\right)$

$v_{3}\left(p, x_{3}, \sigma^{2}\right)$ is also given by (4.31) with $x_{3}(|p|)$ instead of $x_{2}(|p|)$. Estimation of $v_{3}\left(p, x_{3}, \sigma^{2}\right)$ is very simple because we have $\xi_{S}^{\prime}\left(\sigma^{2}\right) \geq \sigma / c s$. In fact $v_{3}\left(., ., \sigma^{2}\right)$ verifies (4.18) with $\delta=s_{2}+1 / 2$.

As for the remarks 2.7 and 2.8 in [6] we have $R_{2}^{+}\left(\sigma^{2}\right) g_{2}=R_{2}^{-}\left(\sigma^{2}\right) g_{2}$.

This concludes the proof of proposition 4.2.

Q.E.D. 
4.2. Study_of $R_{1}\left(\underline{\sigma}_{-}^{2}\right) g_{-}^{\prime}$

Let $\eta$ be a strictly positive number such that $\sigma / c_{S}+\eta<\sigma / c_{R}-\eta$. Let $x_{1}$ (resp. $x_{2}, x_{3}, x_{4}, x_{5}, x$ ) be the characteristic function of $\left(0, \sigma / c_{P}\right)$ (resp. $\left(\sigma / c_{P}, \sigma / c_{S}\right),\left(\sigma / c_{S}, \sigma / c_{S}+\eta\right),\left(\sigma / c_{S}+\eta, \sigma / c_{R}-\eta\right) \cup\left(\sigma / c_{R}+n, \infty\right)$, $\left(\sigma / c_{R}-\eta, \sigma / c_{R}+\eta\right),(0, \infty) \backslash\left[\sigma / c_{R}-\eta, \sigma / c_{R}+n\right]$.

Let

$$
w_{i}\left(p, x_{3}, z\right)=\chi_{i}(|p|) R_{1}(z){ }^{t}\left(g_{1}(p, .), g_{3}(p, .)\right)\left(x_{3}\right)
$$

where $z \in \mathbb{C} \backslash[0, \infty)$ and $i=1,2,3,4,5$, and

$$
w\left(p, x_{3}, \sigma^{2}\right)=\chi(|p|) R_{1}^{+}\left(\sigma^{2}\right){ }^{t}\left(g_{1}(p, .), g_{3}(p, .)\right)\left(x_{3}\right) .
$$

We then have

\section{Proposition 4.3}

Assume $s_{1}>1 / 2$ and $s_{2}>1 / 2$. Let $f \in L^{2 ; s_{1}, s_{2}}\left(\mathbb{R}_{+}^{3}, \mathbb{C}^{3}\right)$ and let $\sigma$ be a strictly positive number such that

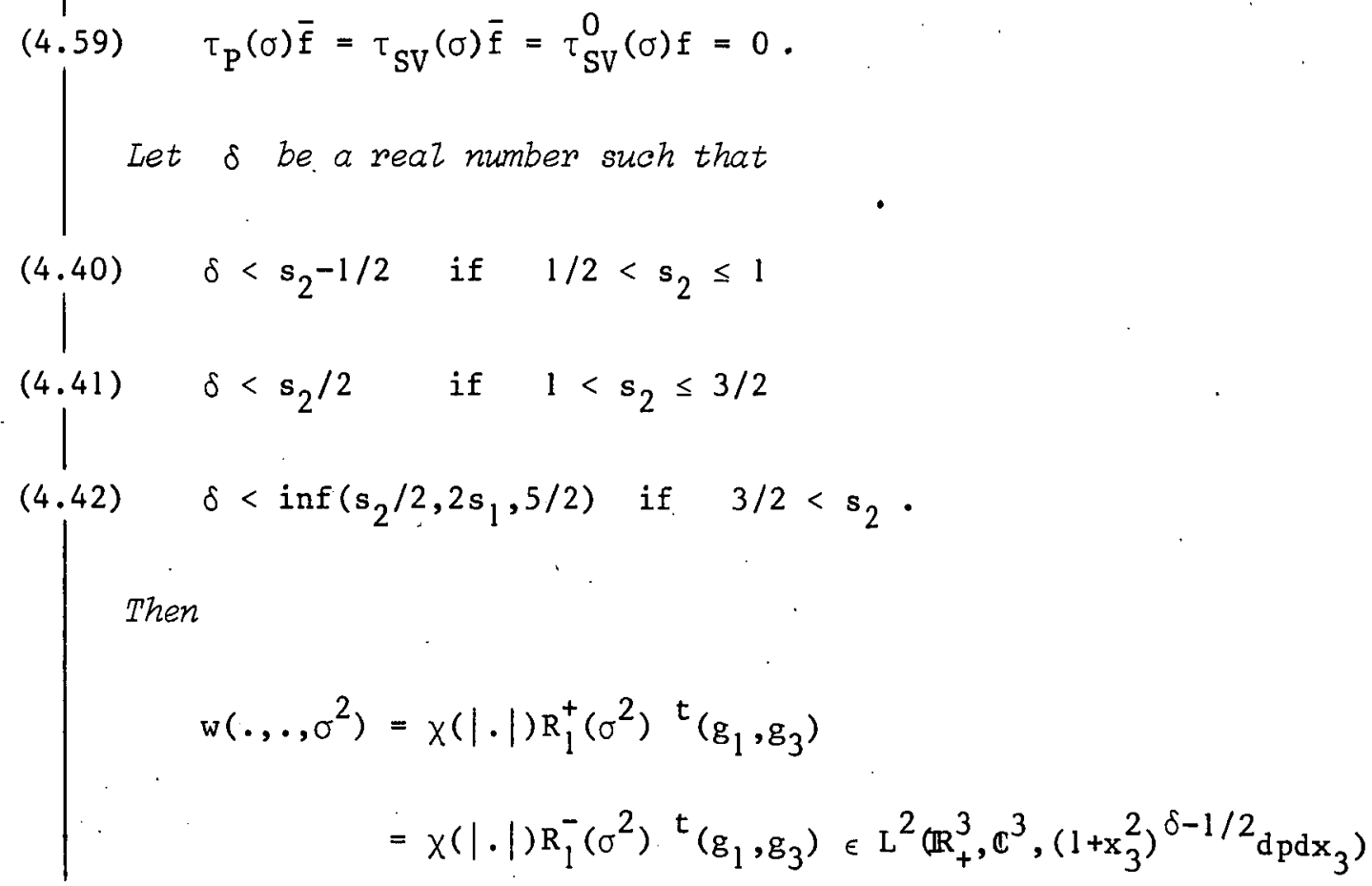




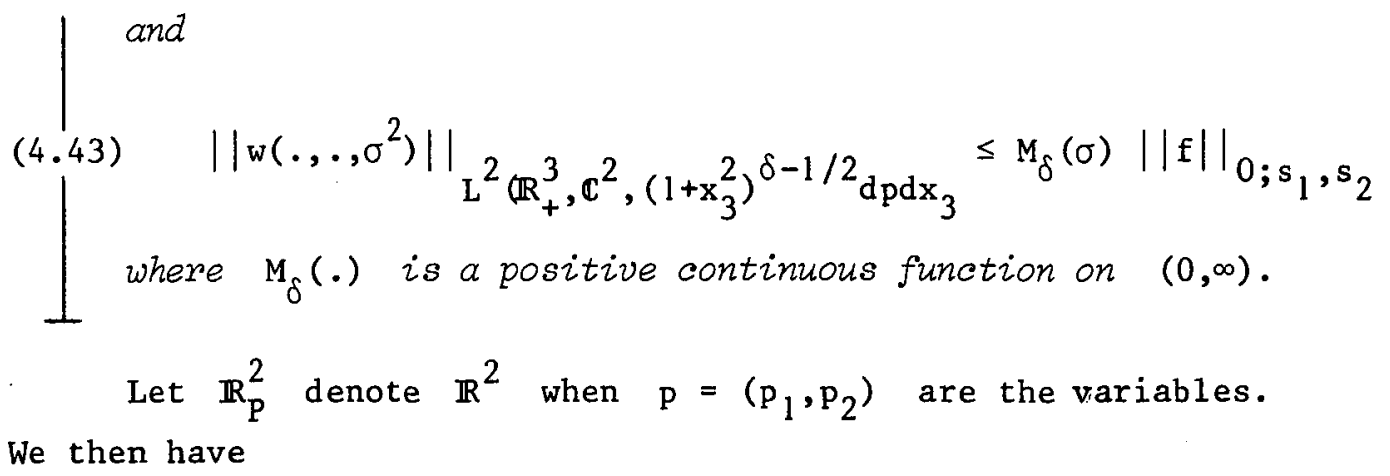

\section{Proposition 4.4}

Suppose $\mathrm{s}_{1}>1 / 2$ and $\mathrm{s}_{2}>1 / 2$. Let $\mathrm{f} \in \mathrm{L}^{2 ; \mathrm{s}_{1}, \mathrm{~s}_{2}}\left(\mathbb{R}_{+}^{3}, \mathbb{C}^{3}\right)$ and let

$\sigma$ be a strictly positive number such that

$$
\int_{\text {Let } \delta \text { be a real number such that } \delta<s_{2} \text {. }}^{(4.44)} \tau_{\mathrm{R}}(\sigma) \mathrm{f}=0 .
$$

Then

$$
\mathrm{w}_{5}\left(., ., \sigma^{2}\right) \in \mathrm{H}^{\inf \left(\mathrm{s}_{1}-1,0\right)}\left(\mathbb{R}_{\mathrm{P}}^{2}, \mathrm{~L}^{2 ; \delta-1 / 2}\left(\mathbb{R}_{+}, \mathbb{C}^{2}, \mathrm{dx}_{3}\right)\right)
$$

$$
\underbrace{(4.45)}_{\mathrm{H}}|| \mathrm{w}_{5}\left(., ., \sigma^{2}\right)\left\|\inf \left(\mathrm{s}_{1}-1,0\right) \underset{\left.\mathbb{R}_{\mathrm{P}}^{2}, \mathrm{~L}^{2 ; \delta-1 / 2}\left(\mathbb{R}_{+}, \mathbb{C}^{2}, \mathrm{dx}_{3}\right)\right)}{ } \leq \mathrm{M}_{\delta}(\sigma)|| \mathrm{f}\right\|_{0 ; \mathrm{s}_{1}, \mathrm{~s}_{2}}
$$

where $\mathrm{M}_{\delta}($.$) is a positive continuous function on (0, \infty)$.

As a consequence of $(4.18),(4.43)$ and $(4.45)$ we have

$$
\begin{gathered}
\chi_{5}(|p|) \Pi\left(R_{1}^{+}\left(\sigma^{2}\right) g^{\prime}(p, .) \oplus R_{2}^{+}\left(\sigma^{2}\right) g_{2}(p, .)\right)\left(x_{3}\right) \\
\in \text { H }^{\inf \left(s_{1}-1,0\right)}\left(\mathbb{R}_{\mathrm{P}}^{2}, L^{2 ; \delta-1 / 2}\left(\mathbb{R}_{+}, \mathbb{C}^{2}, \mathrm{dx}_{3}\right)\right)
\end{gathered}
$$

and (4.7) will enable us to show that

$$
\begin{gathered}
\mathscr{F}^{-1}\left[u\left(p_{1}, p_{2}\right) x_{5}(|p|) \Pi\left(R_{1}^{+}\left(\sigma^{2}\right) g^{\prime}(p, .) \oplus R_{2}^{+}\left(\sigma^{2}\right) g_{2}(p, .)\right)\right]\left(x_{1}, x_{2}, x_{3}\right) \\
\epsilon^{2 ; \tilde{s}_{1}, \delta-1 / 2}\left(\mathbb{R}_{+}^{3}, \mathbb{C}^{3}\right)
\end{gathered}
$$


where $\tilde{s}_{1}$ is defined in theorem 4.1 .

Thus theorem 4.1 will be a consequence of propositions 4.2-4.4.

Let $b_{1}(|p|)$ be the $2 \times 2$ system of ordinary differential operators given by the r.h.s. of (3.12). The $w_{i}\left(.,,, \sigma^{2}\right)$ depend on solutions of $b_{1}(|p|) \phi=\sigma^{2} \phi$.

More precisely let

$$
\phi_{\mathrm{P}}\left(\mathrm{x}_{3},|\mathrm{p}|, \sigma^{2}\right)=2 \pi \mathrm{e}^{-\mathrm{i}|\mathrm{p}| \mathrm{x}_{1}}\left(\sigma \rho_{0} \xi_{\mathrm{P}}\left(\sigma^{2}\right)\right)^{1 / 2} \psi_{\mathrm{P}}\left(\left(\mathrm{x}_{1}, \mathrm{x}_{3}\right),|\mathrm{p}|, \sigma\right)
$$

where $\psi_{\mathrm{P}}\left(\left(\mathrm{x}_{1}, \mathrm{x}_{3}\right),|\mathrm{p}|, \sigma\right)$ is given by $(3: 70)$

$$
\phi_{S}\left(x_{3},|p|, \sigma^{2}\right)=2 \pi e^{-i|p| x_{1}}\left(\sigma \rho_{0} \xi_{S}\left(\sigma^{2}\right)\right)^{1 / 2} \psi_{S}\left(\left(x_{1}, x_{3}\right),|p|, \sigma\right)
$$

where $\psi_{S}\left(\left(x_{1}, x_{3}\right),|p|, \sigma\right)$ is given by (3.71)

$$
\phi_{S}^{0}\left(x_{3},|p|, \sigma^{2}\right)=2 \pi e^{-i|p| x_{1}}\left(\sigma \rho_{0} \xi_{S}\left(\sigma^{2}\right)\right)^{1 / 2} \psi_{S}^{0}\left(\left(x_{1}, x_{2}\right),|p|, \sigma\right)
$$

where $\psi_{S}^{0}\left(\left(x_{1}, x_{3}\right),|p|, \sigma\right)$ is given by (3.72)

$$
\Phi_{R}\left(x_{3},|p|\right)=2 \pi e^{-i|p| x_{1}} \psi_{R}\left(\left(x_{1}, x_{3}\right),|p|\right)
$$

where $\psi_{\mathrm{R}}\left(\left(\mathrm{x}_{1}, \mathrm{x}_{3}\right),|\mathrm{p}|\right)$ is given by $(3.73)$.

Remark that the r.h.s. of $(4.48)-(4.51)$ depend only on $\sigma^{2}$.

We have

$$
\begin{aligned}
& \mathrm{b}_{1}(|\mathrm{p}|) \phi_{\mathrm{P}}\left(\mathrm{x}_{3},|\mathrm{p}|, \sigma^{2}\right)=\sigma^{2} \phi_{\mathrm{P}}\left(\mathrm{x}_{3},|\mathrm{p}|, \sigma^{2}\right), \text { etc... } \\
& \mathrm{b}_{1}(|\mathrm{p}|) \phi_{\mathrm{R}}\left(\mathrm{x}_{3},|\mathrm{p}|\right)=\mathrm{c}_{\mathrm{R}}^{2}|\mathrm{p}|^{2} \phi_{\mathrm{R}}\left(\mathrm{x}_{3},|\mathrm{p}|\right) .
\end{aligned}
$$

The following lemma is proved by computation 
Lemma 4.5

Suppose $s_{1}>1 / 2$ and $s_{2}>1 / 2$. Let $f \in \mathrm{L}^{2 ; s_{1}, s_{2}}\left(\mathbb{R}_{+}^{3}, \mathbb{c}^{3}\right)$ and let $\sigma$. be a strictly positive number. Let $\mathrm{g}($.$) be given by (4.8).$ Then

(i) $\tau_{P}(\sigma) \bar{f}=0$ if and only if $\int_{0}^{\infty}\left[\phi_{P 1}\left(x_{3},|p|, \sigma^{2}\right) g_{1}\left(p, x_{3}\right)-\phi_{P 2}\left(x_{3},|p|, \sigma^{2}\right) g_{3}\left(p, x_{3}\right)\right] d x_{3}=0$ for $|p|<\sigma / c_{P}$ (ii) $\tau_{S V}(\sigma) \bar{f}=0$ if and only if $(4.55) \quad \int_{0}^{\infty}\left[\phi_{S 1}\left(x_{3},|p|, \sigma^{2}\right) g_{1}\left(p, x_{3}\right)-\phi_{S 2}\left(x_{3},|p|, \sigma^{2}\right) g_{3}\left(p, x_{3}\right)\right] d x_{3}=0$ for $|p|<\sigma / c_{p}$ (iii) $\tau_{\mathrm{SV}}^{0}(\sigma) \overline{\mathrm{f}}=0$ if and only if

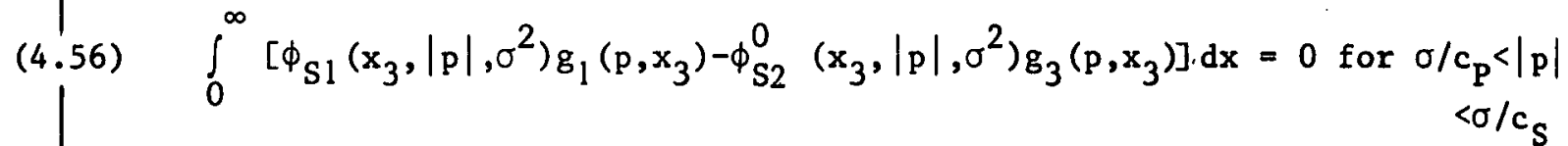
(iv) $\tau_{R}(\sigma) f=0$ if and onzy if $\int_{0}^{\infty}\left[\phi_{R 1}\left(x_{3},|p|\right) g_{1}\left(p, x_{3}\right)+\phi_{R 2}\left(x_{3},|p|\right) g_{3}\left(p, x_{3}\right)\right] d x_{3}=0$ for $|p|=\sigma / c_{R}$.

4.2.1. Definition and estimate of $w_{1}\left(p, x_{3}, \sigma^{2}\right)$

In this section let $\phi_{P}\left(x_{3},|p|, z\right)$ (resp. $\phi_{S}\left(x_{3},|p|, z\right)$ ) be the function we get from (4.48) (resp. (4.49)) by substituting $\xi_{\mathrm{P}}(z)$ (resp. $\xi_{\mathrm{S}}(z)$ ) for $\xi_{\mathrm{P}}(\sigma) \quad\left(\right.$ resp. $\left.\xi_{\mathrm{S}}(\sigma)\right)$.

Let

$$
\Phi_{P}\left(x_{3},|p|, z\right)=e^{i \xi_{P}(z) x_{3} t}\left(|p|, \xi_{P}(z)\right)
$$

$$
\Phi_{S}\left(x_{3},|p|, z\right)=e^{i \xi_{S}(z) x_{3}} t_{\left(\xi_{S}(z),-|p|\right)} \text {. }
$$

All the $\phi$ and $\Phi$ satisfy $b_{1}(|p|) \phi=z \phi$. By adapting the proof 
of the corollary XIII 3.13 in [9] to our case we obtain

$$
w_{1}\left(p, x_{3}, z\right)=\sum_{j=1}^{4} w_{1 j}\left(p, x_{3}, z\right)=\sum_{j=1}^{4} i x_{1}(|p|) \frac{1}{2 z} w_{1 j}^{\prime}\left(p, x_{3}, z\right)
$$

for $\operatorname{Rez}>\mathrm{c}_{\mathrm{P}}^{2}|\mathrm{p}|^{2}$ and $\operatorname{Imz}>0$ where

$$
w_{11}^{1}\left(p, x_{3}, z\right)=\left(\xi_{P}(z)\right)^{-1} \Phi_{P}\left(x_{3},|p|, z\right) \int_{0}^{x_{3}}\left[\phi_{P 1}(y,|p|, z) g_{1}(p, y)\right.
$$

$$
\left.-\phi_{P 2}(y,|p|, z) g_{3}(p, y)\right] d y
$$

$$
w_{12}^{\prime}\left(p, x_{3}, z\right)=\left(\xi_{S}(z)\right)^{-1} \Phi_{S}\left(x_{3},|p|, z\right) \int_{0}^{x_{3}}\left[\phi_{S 1}(y,|p|, z) g_{1}(p, y)\right.
$$

$$
\left.-\phi_{S 2}(y,|p|, z) g_{3}(p, y)\right] d y
$$

$$
w_{13}^{\prime}\left(p, x_{3}, z\right)=\left(\xi_{P}(z)\right)^{-1} \phi_{P}\left(x_{3},|p|, z\right) \int_{x_{3}}^{\infty}\left[\Phi_{P 1}(y,|p|, z) g_{1}(p, y)\right.
$$

$$
\left.-\Phi_{P 2}(y,|p|, z) g_{3}(p, y)\right] d y
$$

$$
w_{14}^{\prime}\left(p, x_{3}, z\right)=\left(\xi_{S}(z)\right)^{-1} \phi_{S}\left(x_{3},|p|, z\right) \int_{x_{3}}^{\infty}\left[\Phi_{S 1}(y,|p|, z) g_{1}(p, y)\right.
$$

$$
\left.-\Phi_{S 2}(\dot{y},|p|, z) g_{3}(p, y)\right] d y
$$

If $z$ tends to $\sigma^{2}$ with a positive imaginary part we get

$$
w_{1}\left(p, x_{3}, \sigma^{2}\right)=\sum_{j=1}^{4} w_{1 j}\left(p, x_{3}, \sigma^{2}\right)=\sum_{j=1}^{4} i x_{1}(|p|) \frac{1}{2 \sigma^{2}} w_{1 j}^{\prime}\left(p, x_{3}, \sigma^{2}\right)
$$

where the $w_{1 j}^{\prime}\left(p, x_{3}, \sigma^{2}\right)$ are given by the r.h.s. of $(4.59)-(4.62)$ but with $\xi_{P}\left(\sigma^{2}\right), \phi_{P}\left(., ., \sigma^{2}\right) \quad \ldots$ instead of $\xi_{P}(z), \phi_{P}(., ., z) \ldots$

Moreover it follows from (4.54) and (4.55) that we have

$$
w_{11}^{\prime}\left(p, x_{3}, \sigma^{2}\right)=-\left(\xi_{P}\left(\sigma^{2}\right)\right)^{-1} \Phi_{P}\left(x_{3},|p|, \sigma^{2}\right) \int_{x_{3}}^{\infty}\left[\phi_{P 1}\left(y,|p|, \sigma^{2}\right) g_{1}(p, y)\right.
$$

$$
\left.-\phi_{P 2}\left(y,|p|, \sigma^{2}\right) g_{3}(p, y)\right] d y
$$




$$
\begin{aligned}
w_{12}^{\prime}\left(p, x_{3}, \sigma^{2}\right) & =-\left(\xi_{S}\left(\sigma^{2}\right)\right)^{-1} \Phi_{S}\left(x_{3},|p|, \sigma^{2}\right) \int_{x_{3}}^{\infty}\left[\phi_{S 1}\left(y,|p|, \sigma^{2}\right) g_{1}(p, y)\right. \\
& \left.-\phi_{S 2}\left(y,|p|, \sigma^{2}\right) g_{3}(p, y)\right] d y .
\end{aligned}
$$

Let us remark that

$$
\left|\phi_{\mathrm{Pi}}\left(\mathrm{y},|\mathrm{p}|, \sigma^{2}\right)\right| \leq \mathrm{c}_{\gamma}\left(\xi_{\mathrm{p}}\left(\sigma^{2}\right)\right)^{\gamma}\left(1+\mathrm{y}^{2}\right)^{\sigma / 2} \text { for } 0 \leq \gamma \leq 1, i=1,2 \text {. }
$$

Thus from $w_{13}^{\prime}\left(p, x_{3}, \sigma^{2}\right), w_{14}^{\prime}\left(p, x_{3}, \sigma^{2}\right),(4.63),(4.64)$ and $(4.65)$ we get

$$
\left.\left|w_{1 j}\left(p, x_{3}, \sigma^{2}\right)\right| \leq C \chi_{l}(|p|)\left(\xi_{P}\left(\sigma^{2}\right)\right)^{\gamma-1}\left(1+y^{2}\right)^{-\tau / 2}\left(\int_{0}^{\infty}\left(1+y^{2}\right)^{s}{ }^{2}|g(p, y)|^{2} d y\right)\right)^{1 / 2}
$$

if $0 \leq \gamma \leq 1$ and $\tau<s_{2}-\gamma-1 / 2$.

Finally $w_{1}\left(p, x_{3}, \sigma^{2}\right)$ satisfies (4.43) with $\delta<s_{2}-1 / 2$.

\subsubsection{Definition and estimate of $w_{2}\left(p ; x_{3}, \sigma^{2}\right)$}

We now consider analytic extensions of the functions $\phi\left(x_{3},|p|, z\right)$ and $\Phi\left(x_{3},|p|, z\right)$ from the upper halfplane $\{\operatorname{Imz}>0\}$ into the lower one $\{\operatorname{Imz}<0\}$ through the interval $\left(c_{S}^{2}|p|^{2}, c_{p}^{2}|p|^{2}\right)$. For that it suffices to substitute $i \xi_{\mathrm{P}}^{\prime}(z)$ for $\xi_{\mathrm{P}}(z)$ in $\phi_{\mathrm{P}}\left(x_{3},|p|, z\right)$, $\phi_{S}\left(x_{3},|p|, z\right), \Phi_{P}\left(x_{3},|p|, z\right)$ given in $4.2 .1 . \phi_{S}\left(x_{3},|p|, z\right)$ remains the same.

We keep the same notations for these analytic extensions as those used in 4.2 .1 but we must remark that with these notations we now have

$$
\phi_{S}\left(x_{3},|p|, \sigma^{2}\right)=\phi_{S}^{0}\left(x_{3},|p|, \sigma^{2}\right)
$$

where $\sigma / c_{\mathrm{P}}<|\mathrm{p}|<\sigma / \mathrm{c}_{\mathrm{S}}$ and where $\phi_{\mathrm{S}}^{0}\left(\mathrm{x}_{3},|\mathrm{p}|, \sigma^{2}\right)$ is given by (4.50).

Thus for $z \in \mathbb{C}$ such that $\mathrm{c}_{\mathrm{S}}^{2}|\mathrm{p}|^{2}<\operatorname{Rez}<\mathrm{c}_{\mathrm{P}}^{2}|\mathrm{p}|^{2}$ and $\operatorname{Imz}>0$ we have 
(4.68) $\quad w_{2}\left(p, x_{3}, z\right)=\sum_{j=1}^{4} w_{2 j}\left(p, x_{3}, z\right)$.

Each $w_{2 j}$ is deduced from the corresponding $w_{1 j}$ defined in 4.2.1 by substituting in it $\chi_{2}(|p|)\left(2 z \xi_{p}^{\prime}(z)\right)^{-1}$ (resp. $\left.i \chi_{2}(|p|)\left(2 z \xi_{s}(z)\right)^{-1}\right)$ for $i \chi_{l}(|p|)\left(2 z \xi_{p}(z)\right)^{-1}$ (resp. i i $\left.\chi_{l}(|p|)\left(2 z \xi_{S}(z)\right)^{-1}\right)$.

As in 4.2 .1 we define $w_{2}\left(p, x_{3}, \sigma^{2}\right)$ and $w_{2 j}\left(p, x_{3}, \sigma^{2}\right) \quad(j=1,2,3,4)$. (4.67) enables us to use (4.56) in order to transform $w_{22}$ as we have done for $w_{11}$ and $w_{12}$ in 4.2 .1 . We have

(4.69) $\left|\phi_{\mathrm{Pi}}\left(\mathrm{x}_{3},|\mathrm{p}|, \sigma\right)\right| \leq \mathrm{c}_{\gamma}\left(\xi_{\mathrm{p}}^{\prime}\left(\sigma^{2}\right)\right)^{\gamma}\left(1+\mathrm{x}_{3}^{2}\right)^{\gamma / 2} \mathrm{e}^{\xi_{\mathrm{P}}^{\prime}\left(\gamma^{2}\right) \mathrm{x}_{3}}$

(4.70) $\left|\phi_{S i}\left(x_{3},|p|, \sigma\right)\right| \leq c_{\gamma}\left(\xi_{S}\left(\sigma^{2}\right)\right)^{\gamma}\left(1+x_{3}^{2}\right)^{\gamma / 2}$

for $i=1,2$ and $0 \leq \gamma \leq 1$.

(4.69) and (4.70) enable us to estimate each $w_{2 j}(j=2,3,4)$ as $\mathrm{w}_{1 \mathrm{j}}$ in 4.2 .1 .

$\mathrm{w}_{21}$ needs a more careful study, especially when $s_{2}>1$.

First of all for $s_{2}, \delta$ and $\gamma$ such that $1 / 2<s_{2} \leq 1, \delta<s_{2}-1 / 2$ and $0 \leq \gamma \leq 1$ one has

$$
\left|\mathrm{w}_{21}\left(\mathrm{p}, \mathrm{x}_{3}, \sigma^{2}\right)\right|\left(1+\mathrm{y}^{2}\right)(\delta-1 / 2) / 2 \leq
$$

$(4.71)$

$$
\frac{C x_{2}(|p|)}{\left(\xi_{p}^{\prime}\left(\sigma^{2}\right)\right)^{1-\gamma}} \int_{0}^{x_{3}} \frac{e^{-\xi_{p}^{\prime}\left(\sigma^{2}\right)\left(x_{3}-y\right)}}{\left(1+\left|x_{3}-y\right|^{2}\right)^{(1 / 2-\gamma) / 2}}\left(1+y^{2}\right)^{\gamma / 2}|g(p, y)| d y
$$

and in that case $w_{21}$ has the same behaviour as $w_{2 j} \quad(j=2,3,4)$.

When $s_{2}>1$ we proceed in the same way as we have done for $\mathrm{v}_{2}\left(\mathrm{x}_{3},|\mathrm{p}|, \sigma\right)$ in 4.1 .2 . 
Let $\varepsilon$ be a strictly positive sufficiently small number such that $\sigma / \mathrm{c}_{\mathrm{P}}+\varepsilon<\sigma / \mathrm{c}_{\mathrm{S}}$. Suppose $\sigma / \mathrm{c}_{\mathrm{P}}<|\mathrm{p}|<\sigma / \mathrm{c}_{\mathrm{P}}+\varepsilon$ and let

$$
p^{\prime}=p|p|^{-1}\left(\sigma^{2} c_{p}^{-2}-\left(\xi_{p}\left(\sigma^{2}\right)\right)^{2}\right)^{1 / 2}=p|p|^{-1}\left(2 \sigma^{2} c_{p}^{-2}-|p|^{2}\right)^{1 / 2} .
$$

We have $\left|\mathrm{p}^{\prime}\right|<\sigma / \mathrm{c}_{\mathrm{P}}$.

Let

$$
\begin{aligned}
& \text { (4.73) } \xi_{\mathrm{P}}\left(\sigma^{2},\left|\mathrm{p}^{\prime}\right|\right)=\left(\sigma^{2} / \mathrm{c}_{\mathrm{P}}^{2}-\left|\mathrm{p}^{\prime}\right|^{2}\right)^{1 / 2}, \xi_{\mathrm{P}}^{\prime}\left(\sigma^{2},|\mathrm{p}|\right)=\left(|\mathrm{p}|^{2}-\sigma^{2} / \mathrm{c}_{\mathrm{P}}^{2}\right)^{1 / 2} \\
& \text { (4.74) } \xi_{\mathrm{S}}\left(\sigma^{2},|\mathrm{p}|\right)=\left(\sigma^{2} / \mathrm{c}_{\mathrm{S}}^{2}-|\mathrm{p}|^{2}\right)^{1 / 2}, \xi_{\mathrm{S}}\left(\sigma^{2},\left|\mathrm{p}^{\prime}\right|\right)=\left(\sigma^{2} / \mathrm{c}_{\mathrm{S}}^{2}-\left|\mathrm{p}^{\prime}\right|^{2}\right)^{1 / 2} .
\end{aligned}
$$

Let

(4.75) $\quad \tilde{\phi}\left(\mathrm{x}_{3},|\mathrm{p}|,\left|\mathrm{p}^{\prime}\right|, \sigma^{2}\right)=\mathrm{id}\left(|\mathrm{p}|,\left|\mathrm{p}^{\prime}\right|, \sigma^{2}\right) \phi_{\mathrm{p}}\left(\mathrm{x}_{3},\left|\mathrm{p}^{\prime}\right|, \sigma^{2}\right)$

where

$$
d\left(|p|,\left|p^{\prime}\right|, \sigma^{2}\right)=\frac{\left(\sigma^{2} / c^{2} s^{-2}\left|p^{\prime}\right|^{2}\right)^{2}+4\left|p^{\prime}\right|^{2} \xi_{p}\left(\sigma^{2},\left|p^{\prime}\right|\right) \xi_{S}\left(\sigma^{2},\left|p^{\prime}\right|\right)}{\left(\sigma^{2} / c^{2} s^{2}|p|^{2}\right)^{2}+4 i|p|^{2} \xi_{p}^{\prime}\left(\sigma^{2},|p|\right) \xi_{S}\left(\sigma^{2},|p|\right)} .
$$

It follows from (4.54) that $w_{21}$ can be written as

$$
\begin{aligned}
w_{21}\left(p, x_{3}, \sigma^{2}\right) & =\sum_{j=1}^{5} w_{21, j}\left(p, x_{3}, \sigma^{2}\right) \\
& =\sum_{j=1}^{5} x_{2}(|p|)\left(2 \sigma^{2} \xi_{p}^{\prime}\left(\sigma^{2},|p|\right)\right)^{-1} w_{21, j}^{\prime}\left(p, x_{3}, \sigma^{2}\right)
\end{aligned}
$$

where

$$
\begin{aligned}
w_{21,1}^{\prime}\left(p, x_{3}, \sigma^{2}\right) & =\Phi_{P}\left(x_{3},|p|, \sigma^{2}\right) \int_{0}^{x_{3}}\left[\phi_{P 1}\left(y,|p|, \sigma^{2}\right)\right. \\
& \left.-\tilde{\phi}_{1}\left(y,|p|,\left|p^{\prime}\right|, \sigma^{2}\right)\right] g_{1}(p, y) d y
\end{aligned}
$$




$$
w_{21,2}^{\prime}\left(p, x_{3}, \sigma^{2}\right)=\Phi_{p}\left(x_{3},|p|, \sigma^{2}\right) \int_{0}^{x_{3}}\left[\tilde{\phi}_{2}\left(y,|p|,\left|p^{\prime}\right|, \sigma^{2}\right)\right.
$$

$$
\left.-\phi_{\mathrm{P} 2}\left(y,|p|, \sigma^{2}\right)\right] g_{3}\left(p^{\prime}, y\right) d y
$$

$$
w_{21,3}^{\prime}\left(p, x_{3}, \sigma^{2}\right)=\Phi_{P}\left(x_{3},|p|, \sigma^{2}\right) \int_{0}^{x_{3}} \tilde{\phi}_{1}\left(y,|p|,\left|p^{\prime}\right|, \sigma^{2}\right)
$$

$$
\left(g_{1}(p, y)-g_{1}\left(p^{\prime}, y\right)\right) d y .
$$

$$
w_{21,4}^{\prime}\left(p, x_{3}, \sigma^{2}\right)=\Phi_{P}\left(x_{3},|p|, \sigma^{2}\right) \int_{0}^{x_{3}} \tilde{\phi}_{2}\left(y,|p|,\left|p^{\prime}\right|, \sigma^{2}\right)
$$

$$
\left(g_{3}\left(p^{\prime}, y\right)-g_{3}(p, y)\right) d y
$$

$$
w_{21,5}^{\prime}\left(p, x_{3}, \sigma^{2}\right)=\Phi_{P}\left(x_{3},|p|, \sigma^{2}\right) \int_{x_{3}}^{\infty}\left[\tilde{\phi}_{2}\left(y,|p|,\left|p^{\prime}\right|, \sigma^{2}\right) g_{3}\left(p^{\prime}, y\right)\right.
$$

$$
\left.-\tilde{\phi}_{1}\left(y,|p|,\left|p^{\prime}\right|, \sigma^{2}\right) g_{1}\left(p^{\prime}, y\right)\right] d y \text {. }
$$

Notations used in $(4.77)-(4.81)$ should be clear. For example the r.h.s. of (4.77) is a function of both $|p|$ and $\left|p^{\prime}\right|$ but it can be considered as a function of $p$ alone because of (4.72). In this last case it is denoted by $w_{21,1}^{\prime}\left(p, x_{3}, \sigma^{2}\right)$.

Finally it follows from (4.76)-(4.81) that $w_{21}$ satisfies (4.43) for any $\delta$ such that $\delta<\inf \left(s_{2} / 2,2 s_{1}, 2 s_{1}+s_{2}-3 / 2,5 / 2\right)$ because of the following inequalities

$$
\begin{gathered}
|| p|-| p^{\prime}|| \leq c\left(\xi_{p^{\prime}}^{\prime}\left(\sigma^{2},|p|\right)\right)^{2} \\
\left|\phi_{P_{i}}\left(y,|p|, \sigma^{2}\right)-\phi_{i}\left(y,|p|,\left|p^{\prime}\right|, \sigma^{2}\right)\right| \leq c_{\gamma}\left(\xi_{p}^{\prime}\left(\sigma^{2},|p|\right)^{3 \gamma}\left(1+y^{2}\right)^{3 \gamma / 2}\right. \\
e^{\xi_{P}^{\prime}\left(\sigma^{2},|p|\right) y}
\end{gathered}
$$

$$
\left|\tilde{\phi}_{i}\left(y,|p|,\left|p^{\prime}\right|, \sigma^{2}\right)\right| \leq C_{\gamma}\left(\xi_{P}^{\prime}\left(\sigma^{2},|p|\right)\right)^{\gamma}\left(1+y^{2}\right)^{\gamma / 2}
$$

for $0 \leq \gamma \leq 1$. 
4.2.3. Definition and estimation of $w_{3}\left(p, x_{3}, \sigma^{2}\right)$

In section 4.2 .3 and 4.2 .4 let $\phi_{\mathrm{P}}\left(\mathrm{x}_{3},|\mathrm{p}|, \mathrm{z}\right), \phi_{\mathrm{S}}\left(\mathrm{x}_{3},|\mathrm{p}|, \mathrm{z}\right)$ $\Phi_{P}\left(x_{3},|p|, z\right)$ and $\Phi_{S}\left(x_{3},|p|, z\right)$ be the functions we obtain from (4.48), $(4.50)$ and $(4.58)$ by substituting $\tilde{\xi}_{P}(z)$ and $\tilde{\xi}_{S}(z)$ for $\xi_{p}\left(\sigma^{2}\right)$ and $\xi_{S}\left(\sigma^{2}\right)$

As before we have

$$
w_{3}\left(p, x_{3}, z\right)=\sum_{j=1}^{4} w_{3 j}\left(p, x_{3}, z\right) .
$$

Each $w_{3 j}$ is deduced from the corresponding $w_{1 j}$ by using the new definitions of $\phi_{P}, \phi_{S}, \Phi_{P}, \Phi_{S}$ and by substituting $\tilde{\xi}_{P}(z)$ (resp. $\tilde{\xi}_{S}(z)$, $\chi_{3}(|p|)$ ) for $\xi_{p}(z)$ (resp. $\left.\xi_{S}(z), \chi_{1}(|p|)\right)$. When $z$ tends to $\sigma^{2}$ with a positive imaginary part, we define $w_{3}\left(p, x_{3}, \sigma^{2}\right)$ and $w_{3 j}\left(p, x_{3}, \sigma^{2}\right)$.

We now have $(i=1,2)$

$$
\left|\Phi_{\mathrm{Pi}}\left(\mathrm{x}_{3},|\mathrm{p}|, \sigma^{2}\right)\right| \leq \mathrm{C} \mathrm{e}^{-\xi_{\mathrm{P}}^{\prime}\left(\sigma^{2}\right) \mathrm{x}_{3}}
$$

$(4.86)$

$$
\begin{aligned}
& \left|\phi_{\mathrm{Pi}}\left(\mathrm{x}_{3},|\mathrm{p}|, \sigma^{2}\right)\right| \leq \mathrm{C} \mathrm{e}^{\xi_{\mathrm{P}}^{\prime}\left(\sigma^{2}\right) \mathrm{x}_{3}} \\
& \xi_{\mathrm{P}}^{\prime}\left(\sigma^{2}\right) \geq \mathrm{C}>0
\end{aligned}
$$

(4.86) enables us to show that $w_{31}\left(p, x_{3}, \sigma^{2}\right)$ and $w_{33}\left(p, x_{3}, \sigma^{2}\right)$ both satisfy (4.43) with $\delta=s_{2}+1 / 2$. Furthermore we have

$$
\left|\Phi_{S i}\left(x_{3},|p|, \sigma^{2}\right)\right| \leq C e^{-\xi_{S}^{\prime}\left(\sigma^{2}\right) x_{3}}
$$

$$
\left|\phi_{S i}\left(x_{3},|p|, \sigma^{2}\right)\right| \leq c_{\gamma}\left(\xi_{S}^{\prime}\left(\sigma^{2}\right)\right)^{\gamma}\left(1+x_{3}^{2}\right)^{\gamma / 2} e^{\xi_{S}^{\prime}\left(\sigma^{2}\right) x_{3}}
$$

with $0 \leq \gamma \leq 1$. 
(4.87) enables us to show that $w_{34}\left(p, x_{3}, \sigma^{2}\right)$ satifies (4.43) for any $\delta$ such that $\delta<s_{2}-1 / 2$.

When $1 / 2<s_{2} \leq 1$ these estimates show that $w_{32}\left(p, x_{3}, \sigma^{2}\right)$

satisfies (4.43) for any $\delta$ such that $\delta<s_{2}-1 / 2$. When $s_{2}>1$ the study of $w_{32}$ is the same as for $w_{21}$ in 4.2.2. In the same

way we consider a strictly positive sufficiently small number $\varepsilon$ and suppose $\sigma / \mathrm{c}_{\mathrm{S}}<|\mathrm{p}|<\sigma / \mathrm{c}_{\mathrm{S}}+\varepsilon$. Let

(4.88) $\quad p^{\prime}=p|p|^{-1}\left(2 \sigma^{2} / c_{s}^{2}-|p|^{2}\right)^{1 / 2}=p|p|^{-1}\left(\sigma^{2} / c_{S}^{2}-\left(\xi_{S}^{\prime}\left(\sigma^{2}\right)\right)^{2}\right)^{1 / 2}$

(4.89) $\quad \xi_{S}^{\prime}\left(\sigma^{2},|\mathrm{p}|\right)=\left(|\mathrm{p}|^{2}-\sigma^{2} / \mathrm{c}_{S}^{2}\right)^{1 / 2}, \xi_{S}\left(\sigma^{2},\left|\mathbf{p}^{\prime}\right|\right)=\left(\sigma^{2} / \mathrm{c}_{S}^{2}-\left|\mathbf{p}^{\prime}\right|^{2}\right)^{1 / 2}$

(4.90) $\quad \xi_{p}^{\prime}\left(\sigma^{2},|p|\right)=\left(|p|^{2}-\sigma^{2} / c_{p}^{2}\right)^{1 / 2}, \xi_{p}^{\prime}\left(\sigma^{2},\left|p^{\prime}\right|\right)=\left(\left|p^{\prime}\right|^{2}-\sigma^{2} / c_{p}^{2}\right)^{1 / 2}$.

Let

(4.91) $\quad \tilde{\phi}^{0}\left(x_{3},|p|,\left|p^{\prime}\right|, \sigma^{2}\right)=i d\left(|p|,\left|p^{\prime}\right|, \sigma^{2}\right) \phi_{. S}^{0}\left(x_{3},\left|p^{\prime}\right|, \sigma^{2}\right)$

where

(4.92) $\mathrm{d}\left(|\mathrm{p}|,\left|\mathrm{p}^{\prime}\right|, \sigma^{2}\right)=\frac{\left(\sigma^{2} / \mathrm{c}^{2}-2\left|\mathrm{p}^{\prime}\right|^{2}\right)^{2}+4 i\left|\mathrm{p}^{\prime}\right|^{2} \xi_{\mathrm{p}^{\prime}}\left(\sigma^{2},\left|\mathrm{p}^{\prime}\right|\right) \xi_{\mathrm{S}}\left(\sigma^{2},\left|\mathrm{p}^{\prime}\right|\right)}{\left(\sigma^{2} / \mathrm{c}_{\mathrm{S}}^{2}-2 \mid \mathrm{p}^{2}\right)^{2}-4|\mathrm{p}|^{2} \xi_{\mathrm{p}}^{\prime}\left(\sigma^{2},|\mathrm{p}|\right) \xi_{\mathrm{S}}^{\prime}\left(\sigma^{2},|\mathrm{p}|\right)}$.

Remark that $\tilde{\phi}^{0}\left(x_{3},|p|,\left|p^{\prime}\right|, \sigma^{2}\right)$ satisfies (4.56). Furthermore we have

$$
\begin{aligned}
\mid \tilde{\phi}_{i}^{0}\left(x_{3},|p|,\left|p^{\prime}\right|, \sigma^{2}\right)-\phi_{S i}\left(x_{3},|p|, \sigma^{2}\right) \leq & C_{\gamma}\left(\xi_{S}^{\prime}\left(\sigma^{2},|p|\right)\right)^{3 \gamma} \cdot\left(1+x_{3}^{2}\right)^{3 \gamma / 2} \\
& e^{\xi_{S}^{\prime}\left(\sigma^{2},|p|\right) x_{3}}
\end{aligned}
$$

when $0 \leq \gamma \leq 1$. 
Finally following the same method as in 4.2 .2 we show that $\mathrm{w}_{32}\left(\mathrm{p}, \mathrm{x}_{3}, \sigma^{2}\right)$ satisfies $(4.43)$ for any $\delta$ such that $\delta<\inf \left(s_{2} / 2,2 s_{1}, 2 s_{1}+s_{2}-3 / 2,5 / 2\right)$ when $s_{2}>1$.

\subsubsection{Definition and estimate of $w_{4}\left(p, x_{3}, \sigma^{2}\right)$}

The study of $w_{4}\left(p, x_{3}, \sigma^{2}\right)$ is very easy because when $\chi_{4}(|p|)$ is different from zero, $\sigma^{2}$ belongs to the resolvent set of $B_{1}(|p|)$ and $\xi_{P}^{\prime}\left(\sigma^{2}\right)$ and $\xi_{S}^{\prime}\left(\sigma^{2}\right)$ are larger than a strictly positive constant. $w_{4}\left(p, x_{3}, z\right)$ can be written in the same way as $w_{3}\left(p, x_{3}, z\right)$ with $x_{4}$ instead of $x_{3}$. Finally we verify that $w_{4}\left(p, x_{3}, \sigma^{2}\right)$ satisfies (4.43) for $\delta=s_{2}+1 / 2$.

4.2.5. $x(1 \cdot 1) \underline{R}_{-1}^{+}\left(\sigma_{-}^{2}\right){ }_{-}^{t}\left(g_{12} g_{2}\right) \equiv x(1 \cdot 1) \underline{R}_{1}^{-}\left(\sigma^{2}\right){ }_{-}^{t}\left(g_{1}, g_{2}\right)$

is proved as in [6] (see remarks 2.7 and 2.8 ).

This concludes the proof of proposition 4.3 .

Q.E.D.

\subsubsection{Proof of_proposition_ 4.4}

$\mathrm{w}_{5}$ is dealt with in the same way as $\tilde{u}_{4}(\mathrm{p}, \mathrm{y}, \mu)$ in section III.4 of [6] (see (3.138)). Recall (4.9) and (4.51).

Let

$$
w_{5}\left(p, x_{3}, z\right)=x_{5}(|p|) \tilde{g}_{R}^{\prime}(p) \phi_{R}\left(x_{3},|p|\right)\left(c_{R}^{2}|p|^{2}-z\right)^{-1}+u\left(p, x_{3}, z\right)
$$

where

$$
u\left(p, x_{3}, z\right)=w_{5}\left(p, x_{3}, z\right)-\lim _{z^{\prime} \rightarrow c_{R}^{2}|p|^{2}}\left(c_{R}^{2}|p|^{2}-z^{\prime}\right)\left(c_{R}^{2}|p|^{2}-z\right)^{-1} w_{5}\left(p, x_{3}, z^{\prime}\right)
$$

When $z$ is not equal to $c_{R}^{2}|p|^{2}, z$ is in the resolvent set of $B_{l}(|p|)$ 
and $w_{5}\left(p, x_{3}, z\right)$ can be written in the same way as $w_{3}\left(p, x_{3}, z\right)$ (see 4.2 .3 ) but with $x_{5}$ instead of $x_{3}$.

Let

(4.96) $\quad D(z)=\left(z / c_{S}^{2}-2|p|^{2}\right)^{2}-4|p|^{2} \xi_{p}^{\prime}(z) \xi_{S}^{\prime}(z)=\left(z-c_{R}^{2}|p|^{2}\right) D^{\prime}(z)$.

Note that $D^{\prime}\left(c_{R}^{2}|p|^{2}\right) \neq 0$.

Let

$$
\Gamma_{\mathrm{P}}\left(\mathrm{x}_{3},|\mathrm{p}|, \mathrm{z}\right)=\mathrm{D}(\mathrm{z}) \phi_{\mathrm{P}}\left(\mathrm{x}_{3},|\mathrm{p}|, \mathrm{z}\right)
$$

(4.97)

$$
\Gamma_{S}\left(x_{3},|p|, z\right)=D(z) \phi_{S}\left(x_{3},|p|, z\right)
$$

where $\phi_{\mathrm{p}}\left(\mathrm{x}_{3},|\mathrm{p}|, z\right)$ and $\phi_{\mathrm{S}}\left(\mathrm{x}_{3},|\mathrm{p}|, z\right)$ are defined in 4.2.3.

$u\left(p, x_{3}, z\right)$ can be written as follows

(4.98) $u\left(p, x_{3}, z\right)=\sum_{j=1}^{4} u_{j}\left(p, x_{3}, z\right)$

where

(4.99) $\quad u_{j}\left(p, x_{3}, z\right)=x_{5}(|p|)\left(2\left(z-c_{R}^{2}|p|^{2}\right)\right)^{-1}\left(u_{j 1}^{\prime}\left(p, x_{3}, z\right)-u_{j 2}^{\prime}\left(p, x_{3}, z\right)\right)$.

Each $u_{j i}^{\prime}$ is defined as follows. Let

$(4 \cdot 100)$

$$
e_{P}(z)=\left(z \xi_{P}^{\prime}(z) D^{\prime}(z)\right)^{-1}
$$

$$
e_{S}(z)=\left(z \xi_{S}^{\prime}(z) D^{\prime}(z)\right)^{-1}
$$

Then 


$$
u_{11}^{\prime}\left(p, x_{3}, z\right)=\int_{0}^{x_{3}}\left[e_{P}(z) \Phi_{P}\left(x_{3},|p|, z\right) \Gamma_{P 1}(y,|p|, z)-e_{P}\left(c_{R}^{2}|p|^{2}\right)\right.
$$

$$
\left.\Phi_{P}\left(x_{3},|p|, c_{R}^{2}|p|^{2}\right) \Gamma_{P 1}\left(y,|p|, c_{R}^{2}|p|^{2}\right)\right] g_{1}(p, y) d y
$$

$u_{12}^{\prime}$ is deduced from $u_{11}^{\prime}$ by substituting $\Gamma_{P 2}$ and $g_{3}$ for $\Gamma_{P 1}$ and $g_{1}$. $u_{21}^{\prime}$ (resp. $u_{22}^{\prime}$ ) is deduced from $u_{11}^{\prime}$ (resp. $u_{12}^{\prime}$ ) by substituting the subseript $S$ for $P$.

Moreover

$$
\begin{aligned}
u_{31}^{\prime}\left(p, x_{3}, z\right)= & \int_{x_{3}}^{\infty}\left[e_{P}(z) \Gamma_{P}\left(x_{3},|p|, z\right) \Phi_{P 1}(y,|p|, z)-\right. \\
& -e_{P}\left(c_{R}^{2}|p|^{2}\right) \Gamma_{P}\left(x_{3},|p|, c_{R}^{2}|p|^{2}\right) \\
& \left.\Phi_{P 1}\left(y,|p|, c_{R}^{2}|p|^{2}\right)\right] g_{1}(p, y) d y
\end{aligned}
$$

$u_{32}^{\prime}$ is deduced from $u_{31}^{\prime}$ by substituting in it $\Phi_{P 2}$ and $g_{3}$ for $\Phi_{\mathrm{P} 1}$ and $\mathrm{g}_{1}$.

$u_{41}^{\prime}$ (resp. $u_{42}^{\prime}$ ) is deduced from $u_{31}^{\prime}$ (resp. $u_{32}^{\prime}$ ) by substituting in it the subscript $S$ for $P$.

$u_{j i}^{\prime}\left(p, x_{3}, \sigma^{2}\right)$ is defined as the limit of $u_{j i}^{\prime}\left(p, x_{3}, z\right)$ when $z$ tends to $\sigma^{2}$ wjth a positive imaginary part. Let

$$
u_{j}\left(p, x_{3}, \sigma^{2}\right)=x_{5}(|p|)\left(2\left(\sigma^{2}-c_{R}^{2}|p|^{2}\right)\right)^{-1}\left(u_{j l}^{\prime}\left(p, x_{3}, \sigma^{2}\right)-u_{j 2}^{\prime}\left(p, x_{3}, \sigma^{2}\right)\right)
$$

(4.104). $u\left(p, x_{3}, \sigma^{2}\right)=\sum_{j=1}^{4} u_{j}\left(p, x_{3}, \sigma^{2}\right)$.

As in theorem 3.6 of [6] one shows that $u\left(p, x_{3}, \sigma^{2}\right)$ satisfies (4.43) with $\delta<s_{2}$.

(4.57) shows that $\tilde{g}_{R}^{\prime}(p)=0$ for $|p|=\sigma / c_{R}$. From this we deduce 
that $\mathrm{w}_{5}\left(., ., \sigma^{2}\right)-\mathrm{u}\left(., ., \sigma^{2}\right)$ belongs to $\mathrm{H}^{\mathrm{s}_{1}-1}\left(\mathbb{R}_{\mathrm{P}}^{2}, \mathrm{~L}^{2, \mathrm{~s}}\left(\mathbb{R}_{+}, \mathbb{C}^{2}, \mathrm{dx} \mathrm{x}_{3}\right)\right)$ for any $s$ by using decreasing properties of $\phi_{R}\left(x_{3},|p|\right.$ ) (see Lemma 3.7 of $[6])$.

This concludes the proof of proposition 4.4 .

\section{LIMITING ABSORPTION PRINCIPLE FOR A}

In this section we give two proofs of the limiting absorption principle for $A$ in appropriate Hilbert spaces. The first one uses the method of D.M. Eidus $([12],[13])$ and the theorem 4.1. The second one uses the method of R. Phillips [22] (see also [20]) and the theorem 4.1 too. Phillips method enables us to prove that the extended resolvents of $A$ are locally Hölder continuous as functions of $z$ with values in bounded operators on appropriate spaces (see theorem 5.2).

Let us mention the main differences with the case of perturbed stratified media [6]. First of all the boundary of $\Omega$ is unbounded for the perturbed isotropic half space with a free boundary. Furthermore if $\psi($.$) is in C_{0}^{\infty}\left(\mathbb{R}^{3}\right)$ and if $\psi(x)=1$ for $|x| \leq L$ and if $u$ is locally in the domain of $A$, it is not necessary true that $\psi_{u}$ is in the domain of $A$ because $\psi \mathbf{u}$ does not necessary satisfy the free boundary condition.

This difficulty is solved by the following lemma.

Lemma 5.1

There exists a bounded Zinear transformation $\mathscr{E}$ of $\mathrm{H}^{3 / 2}\left(\mathbb{R}^{2}, \mathbb{C}^{3}\right)$ into $\mathrm{H}^{3}\left(\mathbb{R}^{3}, \mathbb{C}^{3}\right)$-such that for every $\mathrm{h} \in \mathrm{H}^{3 / 2}\left(\mathbb{R}^{2}, \mathbb{C}^{3}\right)$ the extension $\mathrm{u}=\mathscr{E}, \mathrm{h}$ satisfies 


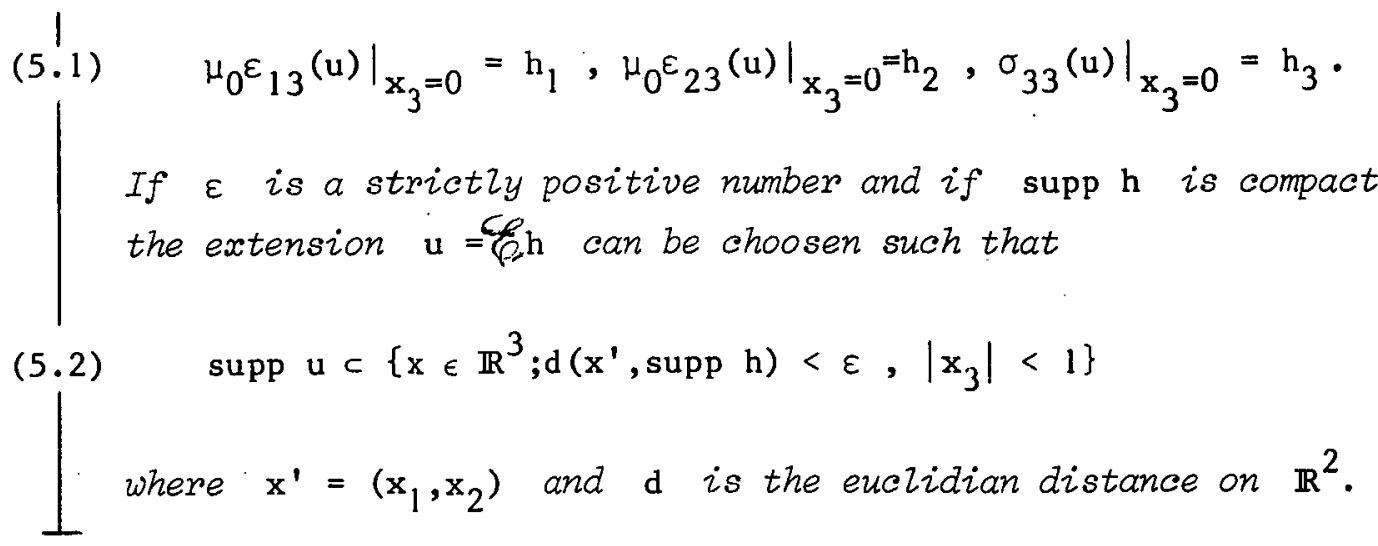

\section{Proof of the lemma}

Let

$$
\gamma_{0}\left(v_{i}\right)=\left.v_{i}\right|_{x_{3}=0}, \quad \gamma_{1}\left(v_{i}\right)=\left.\frac{\partial v_{i}}{\partial x_{3}}\right|_{x_{3}=0}
$$

for $v \in H^{3}\left(\mathbb{R}^{3}, \mathbb{c}^{3}\right)$.

Any such extension $v$ of $h$ must satisfy the following system

$$
\begin{aligned}
& \mu_{0}\left(\gamma_{1}\left(v_{1}\right)+\gamma_{0}\left(\frac{\partial v_{3}}{\partial x_{1}}\right)\right)=h_{1} \\
& \mu_{0}\left(\gamma_{1}\left(v_{2}\right)+\gamma_{0}\left(\frac{\partial v_{3}}{\partial x_{2}}\right)\right)=h_{2} \\
& \lambda_{0} \gamma_{0}\left(\frac{\partial v_{1}}{\partial x_{1}}+\frac{\partial v_{2}}{\partial x_{2}}\right)+\left(\lambda_{0}+2 \mu_{0}\right) \gamma_{1}\left(v_{3}\right)=h_{3} .
\end{aligned}
$$

(5.4) has an infinite number of solutions. In order to choose one of them we add the following conditions

$$
\gamma_{0}\left(v_{1}\right)=\gamma_{0}\left(v_{2}\right)=\gamma_{0}\left(v_{3}\right)=0
$$

Let $\alpha \in C_{0}^{\infty}(\mathbb{R})$ such that $\alpha(0)=0$ and $\alpha^{\prime}(0)=1$. The solution $u$ of $(5.4)$ and $(5.5)$ is given by

$$
\left(\mathscr{B}_{\mathrm{u}}\right)_{i}\left(\mathrm{p}, \mathrm{x}_{3}\right)=\mu_{0}^{-1}\left(1+|p|^{2}\right)^{-1 / 2}\left(\mathscr{F}_{\mathrm{h}}\right)_{i}(p) \alpha\left(\left(1+|p|^{2}\right)^{1 / 2} \mathrm{x}_{3}\right), \quad \mathrm{i}=1,2
$$




$$
(\mathscr{Q u})_{3}\left(p, x_{3}\right)=\left(\lambda_{0}+2 \mu_{0}\right)^{-1}\left(1+|p|^{2}\right)^{-1 / 2}(\not{\not h})_{3}(p) \alpha\left(\left(1+|p|^{2}\right)^{1 / 2} x_{3}\right)
$$

and the transformation $\mathscr{E}$ defined by

(5.8) $\quad \mathscr{E}, \mathrm{h}=\mathrm{u}$

is a bounded operator from $H^{3 / 2}\left(\mathbb{R}^{2}, \mathbf{c}^{3}\right)$ into $\mathrm{H}^{3}\left(\mathbb{R}^{3}, \mathbb{C}^{3}\right)$ such that (5.1) is satisfied.

Moreover if supp $h$ is compact one can choose $\phi \in C_{0}^{\infty}\left(\mathbb{R}^{3}\right)$ such that $\phi \equiv l$ in a sufficiently small neighbourhood of supp $h$, then $\mathscr{C}_{\mathrm{C}}=\phi \mathrm{u}$ satisfies both $(5.1)$ and $(5.2)$.

Let. $s_{1}$ and $s_{2}$ be two real numbers. Let $L^{2 ; s_{1}, s_{2}}\left(\Omega, \mathbb{C}^{3}\right)$ be the space of all measurable $\mathbb{C}^{3}$-valued functions on $\Omega$ defined by

$$
\mathrm{L}^{2 ; \mathrm{s}_{1}, \mathrm{~s}_{2}}\left(\Omega, \mathbb{C}^{3}\right)=\left\{\mathrm{u}(\mathrm{x}):\left(1+\mathrm{x}_{1}^{2}+\mathrm{x}_{2}^{2}\right)^{\mathrm{s}_{1} / 2}\left(1+\mathrm{x}_{3}^{2}\right)^{\mathrm{s}_{2} / 2} \mathrm{u}(\mathrm{x}) \in \mathrm{L}^{2}\left(\Omega, \mathbb{C}^{3}\right)\right\} .
$$

In $L^{2 ; s_{1}, s_{2}}\left(\Omega, \mathbb{C}^{3}\right)$ we introduce the norm

(5.9) $\quad\||| u \mid\|_{0 ; s_{1}, s_{2}}^{2}=\int_{\Omega}\left(1+x_{1}^{2}+x_{2}^{2}\right)^{s}{ }^{1}\left(1+x_{3}^{2}\right)^{s_{2}} u(x) \cdot \overline{u(x)} d x$.

More generally we consider weighted Sobolev $L^{2}$ spaces $H^{m ; s_{1}, s_{2}}\left(\Omega, \mathbb{C}^{3}\right)$ defined for any integer $m \geq 0$ by

(5.10) $\mathrm{H}^{\mathrm{m} ; \mathrm{s}_{1}, \mathrm{~s}_{2}}\left(\Omega, \mathbb{C}^{3}\right)=\left\{\mathrm{u}(\mathrm{x}) ; \mathrm{D}^{\alpha} \mathrm{u} \in \mathrm{L}^{2 ; \mathrm{s}_{1}, \mathrm{~s}_{2}}\left(\Omega, \mathbb{C}^{3}\right), 0 \leq|\alpha| \leq \mathrm{m}\right\}$

$\mathrm{H}^{\mathrm{m} ; \mathrm{s}_{1}, \mathrm{~s}_{2}}\left(\Omega, \mathbb{C}^{3}\right)$ is a Hilbert space under the norm

$$
\left\||u|||_{m ; s_{1}, s_{2}}^{2}=\sum_{|\alpha| \leq m}\right\||| D^{\alpha} u_{1} \|_{0 ; s_{1}, s_{2}}^{2} .
$$

Let 
(5.12) $\quad \mathrm{H}^{1 ; s_{1}, s_{2}}\left(\Omega, \mathcal{A}, \mathbb{C}^{3}\right)=\left\{u \in \mathrm{H}^{1 ; s_{1}, s_{2}}\left(\Omega, \mathbb{C}^{3}\right) ; \mathscr{A} u \in \mathrm{L}^{2 ; \mathbf{s}_{1}, s_{2}}\left(\Omega, \mathbb{C}^{3}\right)\right\}$

where $\mathbb{A}$ is defined by (2.11).

In. $\mathrm{H}^{1 ; \mathrm{s}_{1}, s_{2}}\left(\Omega, \mathbb{A}, \mathbb{C}^{3}\right)$ we introduce the norm

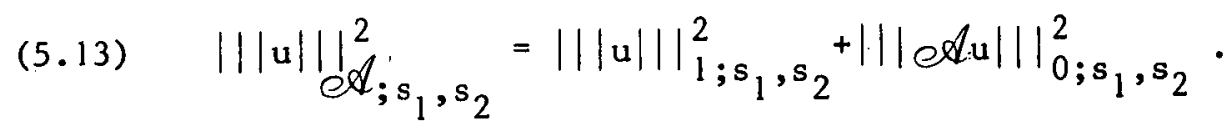

Suppose $s_{1} \geq 0$ and $s_{2} \geq 0$. Let us remark that the 1.h.s. of (2.12) has still a meaning for $u$ in $\mathrm{H}^{1 ;-\mathrm{s}_{1},-\mathrm{s}_{2}}\left(\Omega, \mathcal{A}, \mathrm{c}^{3}\right)$ and $\mathrm{v}$ in $\mathrm{H}^{1 ; \mathrm{s}_{1}, \mathrm{~s}_{2}}\left(\Omega, \mathrm{c}^{3}\right)$. Thus $u$ in $\mathrm{H}^{1 ;-\mathrm{s}_{1},-\mathrm{s}_{2}}\left(\Omega, \mathcal{A}, \mathbb{C}^{3}\right)$ is said to satisfy the generalized free boundary condition if (2.12) is verified for every $v$ in $\mathrm{H}^{1 ; \mathrm{s}_{1}, \mathrm{~s}_{2}}\left(\Omega, \mathbb{C}^{3}\right)$.

Finally let

$$
\mathrm{H}^{1 ;-\mathrm{s}_{1},-\mathrm{s}_{2}}\left(\Omega, \mathrm{A}, \mathbb{C}^{3}\right)=\left\{\mathrm{u} \in \mathrm{H}^{1 ;-\mathrm{s}_{1},-\mathrm{s}_{2}}\left(\Omega, \mathbb{A}, \mathbb{C}^{3}\right) ;\right.
$$

$(5.14)$

u satisfies the generalized free boundary condition\}

$\mathrm{H}^{1 ;-s_{1},-s_{2}}\left(\Omega, A, \mathbb{C}^{3}\right)$ is a closed subspace of $\mathrm{H}^{1 ;-\mathrm{s}_{1},-\mathrm{s}_{2}}\left(\Omega, \mathcal{A}, \mathbb{C}^{3}\right)$.

We then have

Theorem 5.2 (Limiting absorption principle for $A$ )

Suppose $s_{1}>1 / 2$ and $s_{2}>1 / 2$.

(i) For any $\omega_{0}>0$ such that $\omega_{0}^{2}$ is not an eigenvalue for $A$ the following two limits exist in the uniform operator topology of $\mathrm{B}\left(\mathrm{L}^{2 ; \mathrm{s}_{1}, \mathrm{~s}_{2}}\left(\Omega, \mathrm{C}^{3}\right), \mathrm{H}^{1 ;-\mathrm{s}_{1},-\mathrm{s}_{2}}\left(\Omega, \mathrm{A}, \mathrm{C}^{3}\right)\right)$ : 


$$
(5.15) \quad R^{ \pm}\left(\omega_{0}^{2}\right)=\lim _{z \rightarrow \omega_{0}^{2}} R(z)
$$

(ii) For any compact interval $[\mathrm{a}, \mathrm{b}]$ in $(0, \infty)$ which does not contain any eigenvalue of A let

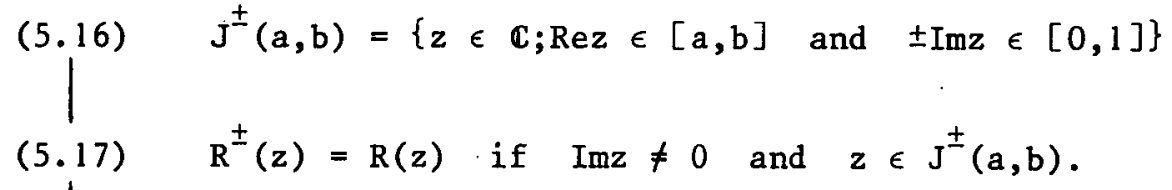

For every $\delta$ such that $\delta<\inf \left(1, s_{1}-1 / 2, s_{2}-1 / 2\right)$ there exists a positive constant $\mathrm{C}$ depending on $\mathrm{A}, \mathrm{a}, \mathrm{b}$, and $\delta$ such that

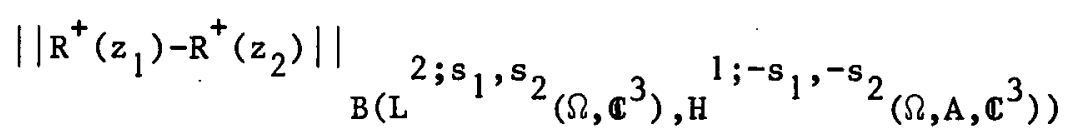

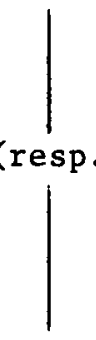

(5.19)

$$
\left.|| \mathrm{R}^{-}\left(\mathrm{z}_{1}\right)-\mathrm{R}^{-}\left(\mathrm{z}_{2}\right)|| \underset{B(\mathrm{~L}}{2 ; \mathrm{s}_{1}, \mathrm{~s}_{2}}\left(\Omega, \mathbb{C}^{3}\right), \mathrm{H}{ }^{1 ;-\mathrm{s}_{1},-\mathrm{s}_{2}}\left(\Omega, A, \mathbb{C}^{3}\right)\right)
$$

$$
\left.\leq \mathrm{C}\left|\mathrm{z}_{1}-\mathrm{z}_{2}\right|^{\delta}\right)
$$

for any $\left(\mathrm{z}_{1}, \mathrm{z}_{2}\right) \in \mathrm{J}^{+}(\mathrm{a}, \mathrm{b}) \times \mathrm{J}^{+}(\mathrm{a}, \mathrm{b})$ (resp. $\left.\mathrm{J}^{-}(\mathrm{a}, \mathrm{b}) \times \mathrm{J}^{-}(\mathrm{a}, \mathrm{b})\right)$.

We will give two differents proofs of (i). The first one uses the method of D.M. Eidus and the second one follows the R. Phillips method. (ii) is proved by using the Phillips one.

\section{First proof}

In order to prove (i) by using the method of D.M. Eidus one knows 
that the main result to be proved (see [30]) is the following inequality

$$
\|\mathrm{H}(\mathrm{z}) \mathrm{f}\|_{\mathbb{A} ;-\mathrm{s}_{1},-\mathrm{s}_{2}} \leq \mathrm{c}\|\mathrm{f}\|_{0 ; \mathrm{s}_{1}, \mathrm{~s}_{2}}
$$

for every $f$ in $L^{2 ; s_{1}, s_{2}}\left(\Omega, c^{3}\right)$ and $z$ in $J^{ \pm}(a, b) \backslash[a, b]$.

Several steps are needed.

\section{Firis $\underline{\text { t_s }}$ teqep}

Suppose $(5.20)$ false. Then there exist sequences $\left(u_{n}\right)$ in $D(A)$, $\left(F_{n}\right)$ in $L^{2 ; s_{1}, s_{2}}\left(\Omega, \mathbb{C}^{3}\right)$ and $\left(z_{n}\right)$ in $J^{ \pm}(a, b) \backslash[a, b]$ such that

$$
z_{n} \text { tends to } \sigma^{2} \in[a, b]
$$

$$
\left\|u_{n}\right\| \|_{d ;-s_{1},-s_{2}}=1
$$

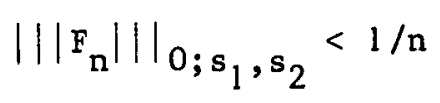

$$
\left(A-z_{n}\right) u_{n}=F_{n}
$$

By choosing $s_{1}^{\prime}>s_{1}$ and $s_{2}^{\prime}>s_{2}$ and by considering, if it is necessary, a subsequence of $\left(u_{n}\right)$ we denote by the same symbol, one then shows that $\left(u_{n}\right)$ converges to a limit, denoted by $u$, in $H^{1 ;-s_{1},-s_{2}}\left(\Omega, A, \mathbb{C}^{3}\right)$. This is a consequence of Korn's inequality and Rellich theorem. Finally we obtain

$$
\left(\mathbb{A}-\sigma^{2}\right) u=0
$$

Let

$$
\Omega^{\prime}=\Omega \cap\left\{\mathbf{x} \in \mathbb{R}^{3} ;|\mathbf{x}|>L\right\} \text {. }
$$

Then $u$ is in $H^{2 ;-s_{j}^{1},-s_{2}^{\prime}}\left(\Omega^{\prime}, c^{3}\right)$ (see [21] p. 222) and the traces of $\varepsilon_{13}(u), \varepsilon_{23}(u)$ and $\sigma_{33}(u)$ on $\Omega^{\prime} n\left\{x \in \mathbf{R}^{3} ; x_{3}=0\right\}$ are well defined and equal to zero. 


\section{Second stepep}

Let $\phi$ be a function in $C^{\infty}\left(\mathbb{R}^{3}\right)$ such that $\phi(x)=1$ for $|x|>L+2$ and $\phi(x)=0$ for $|x|<L+1$. Let

$$
\left.\mu_{0} \varepsilon_{13}(\phi u)\right|_{\mathbf{x}_{3}=0}=h_{1},\left.\mu_{0} \varepsilon_{23}(\phi u)\right|_{x_{3}=0}=h_{2},\left.\sigma_{33}(\phi u)\right|_{x_{3}=0}=h_{3} .
$$

It follows from lemma 5.1 that there exists an extension $\tilde{u}$ of $h$ such that the support of $\tilde{u}$ is compact and contained in $\left\{x \in \mathbb{R}_{+}^{3} ;|x|>L\right\}$.

Let

$$
u^{\prime}=\phi u-\tilde{u}
$$

$\mathrm{u}^{\prime}$ in $\mathrm{H}^{1 ;-\mathrm{s}_{1}^{\prime},-\mathrm{s}_{2}^{\prime}}\left(\Omega, e A, \mathbb{C}^{3}\right)$ satisfies $(2.12)$ for every $\mathrm{v}$ in $\mathrm{H}^{1 ; s_{1}^{j}, \mathrm{~s}_{2}^{\prime}}\left(\Omega, \mathrm{c}^{3}\right)$. Let $\mathscr{A}_{0}$ be the following operator in $\mathscr{Q}\left(\mathbb{R}_{+}^{3}, \mathbb{C}^{3}\right)$ :

$$
\mathscr{A}_{0} u=-\left(\frac{\lambda_{0}+\mu_{0}}{\dot{\rho}_{0}}\right) \nabla(\nabla . u)-\frac{\mu_{0}}{\rho_{0}} \Delta u, u \in \mathscr{D}^{\prime}\left(\mathbb{R}_{+}^{3}, \mathbb{c}^{3}\right) .
$$

Then it follows from (5.25) that $\left(\mathbb{A}_{0}-\sigma^{2}\right) u^{\prime}$ is in $L^{2 ; s ; t}\left(\mathbb{R}_{+}^{3}, \mathbb{C}^{3}\right)$ for any real numbers $s$ and $t$.

From the sequence $\left(z_{n}\right)$ there exists a subsequence we denote by the same symbol such that either $\operatorname{Im} z_{n}>0$ or $\operatorname{Im} z_{n}<0$.

Suppose $\operatorname{Imz}{ }_{n}>0$. It then follows from (5.23), (5.24) and Lemma 5.1 that one has

$$
u^{\prime}=R_{0}^{+}\left(\sigma^{2}\right)\left(A_{0}-\sigma^{2}\right) u^{\prime}
$$

(If $\operatorname{Im}_{\mathrm{n}}<0$ for every $n$ we substitute $\mathrm{R}_{0}^{-}\left(\sigma^{2}\right)$ for $\mathrm{R}_{0}^{+}\left(\sigma^{2}\right)$ in (5.30)) . 


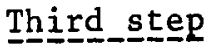

In fact one has (see (3.53))

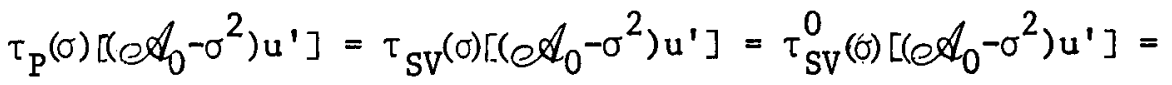

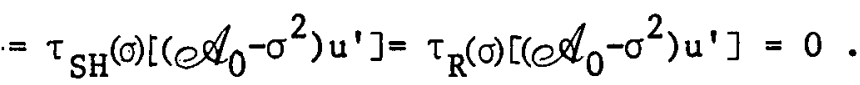

It then follows from (5.30), theorem 4.1 and (5.31) that $u$ ' is in $\mathrm{L}^{2}\left(\mathbb{R}_{+}^{3}, \mathbb{C}^{3}\right)$. Therefore $\mathrm{u}$ is in $\mathrm{L}^{2}\left(\Omega, \mathbb{C}^{3}, \rho(\mathrm{x}) \mathrm{dx}\right)$.

$$
\text { Duality between } \mathrm{L}^{2 ;-\mathrm{s}_{1}^{1}, \mathrm{~s}_{2}^{\prime}}\left(\Omega, \mathbb{C}^{3}\right) \text { and } \mathrm{L}^{2 ; s_{1}^{1}, \mathrm{~s}_{2}^{1}}\left(\Omega, \mathbb{C}^{3}\right) \text { is }
$$

denoted by $\langle\cdot, \cdot\rangle$.

From lemma 3.10 and (5.30) it is sufficient to show that

$$
\left.\left.I=\overline{\left\langle R_{0}^{+}\left(\sigma^{2}\right)\left[\left(\varrho \mathscr{A}_{0}-\sigma^{2}\right) u^{\prime}\right]\right.}, \dot{A}_{0}-\sigma^{2}\right) u^{\prime}\right\rangle=\left\langle\bar{u}^{\prime},\left(e \mathscr{A}_{0}-\sigma^{2}\right) u^{\prime}\right\rangle
$$

is a real number in order to prove (5.31).

Remark that the support of $\left(\mathbb{A}_{0}-\sigma^{2}\right) u^{\prime}$ is contained in $\left\{x \in \mathbb{R}_{+}^{3} ;|x|<L+2\right\}$. Let $\chi$ be a real function in $C_{0}^{\infty}\left(\mathbb{R}^{3}\right)$ such that $X(x)=1$ for $|x|<L+2$. We then have

$$
I=\left\langle\chi \bar{u}^{\prime}, e A_{0} u^{\prime}\right\rangle-\sigma^{2}\left\langle\bar{u}^{\prime}, \chi u^{\prime}\right\rangle
$$

Thus, in order to show that $I$ is a real number it is sufficient to prove that $\left\langle x \bar{u}, e_{0} u^{\prime}\right\rangle$ is real.

$u^{\prime}$ satisfies the generalized free boundary condition. Thus we get

$$
I=\int_{\mathbb{R}_{+}^{3}}\left[\lambda_{0} \nabla \cdot u^{\prime}\left(\bar{u}^{\prime} \cdot \nabla X\right)+\mu_{0} \sum_{i, j=1}^{3} \varepsilon_{i j}\left(u^{\prime}\right)\left(\bar{u}_{i}^{\prime} \frac{\partial x}{\partial x_{j}}+\bar{u}_{j}^{\prime} \frac{\partial x}{\partial x_{i}}\right)\right] d x+G
$$

where $G$ is a real number.

In the r.h.s. of (5.34) we are integrating on the support of $\nabla \chi$. 
Thus one can substitute $u$ for $u^{\prime}$ in (5.34).

But we have $A d u=\sigma^{2} u$ and $u$ is in $\mathrm{H}^{1 ;-s_{1}^{1},-s_{2}^{1}}\left(\Omega, A, \mathbb{C}^{3}\right)$.

Therefore $\langle x \bar{u}$, A tu $\rangle$ is a real number and we have

$$
J=\langle\chi \bar{u} \cdot e A u\rangle=\int_{\Omega}\left[\lambda \nabla \cdot u \nabla \cdot(\chi \bar{u})+2 \mu \sum_{i, j=1}^{3} \varepsilon_{i j}(u) \varepsilon_{i j}(\chi \bar{u})\right] d x
$$

$$
=\int_{\mathbb{R}_{+}^{3}}\left[\lambda \nabla \cdot u(\bar{u} \cdot \nabla \chi)+\mu \sum_{i, j=1}^{3} \varepsilon_{i j}(u)\left(\bar{u}_{i} \cdot \frac{\partial \chi}{\partial x_{j}}+\bar{u}_{j} \frac{\partial \chi}{\partial x_{i}}\right)\right] d x+G^{\prime}
$$

where $G^{\prime}$ is a real number.

One has $\lambda(x)=\lambda_{0}$ and $\mu(x)=\mu_{0}$ for $x$ in the support of $\nabla x$. Furthermore the r.h.s. of (5.35) is real. Therefore $I$ is real too. Thus (5.31) is proved.

Finally we prove that $u$ is different from zero because we have

$$
\|\mid u\|_{d ;-s_{1},-s_{2}}=1 \text {. }
$$

Thus $u$ is an eigenfunction of $A$ and $\sigma^{2}$ in $[a, b]$ is the associated eigenvalue. This is a contradiction because we have supposed that the interval $[a, b]$ does not contain any eigenvalue of $A$.

In order to finish the proof of (i) see [6] or [30].

\section{Second proof}

Let $\overline{\mathbb{C}^{+}}=\{z \in \mathbb{C} ; z \neq 0, \operatorname{Imz} \geq 0\}$. In this new approach we give a construction of $u=R(z) f$ for every $f$ in $L^{2 ; s_{1}, s_{2}}(\Omega)$ and every $z$ in $\overline{\mathbb{C}^{+}}$such that $\operatorname{Imz}>0$.

From this construction it is possible to deduce (i) and (ii). Several steps are needed for proving (i) and (ii) of theorem 5.2. 


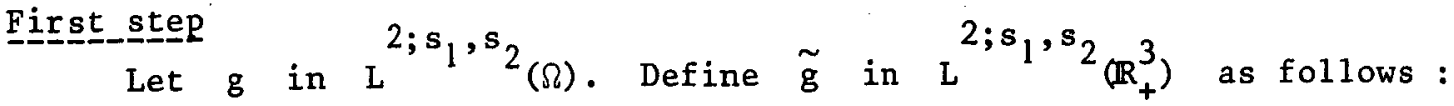

$$
\tilde{g}(x)=g(x) \text { if } \quad x \in \mathbb{R}_{+}^{3} \cap \Omega
$$

$$
\tilde{g}(x)=0 \quad \text { if } \quad x \in \mathbb{R}_{+}^{3} \backslash\left(\mathbb{R}_{+}^{3} \cap \Omega\right) .
$$

Let

$$
\Omega_{+}^{L}=\left\{x \in \Omega ;|x|<L+6, x_{3}>0\right\}
$$

$$
\begin{aligned}
& \Omega_{-}^{L}=\left\{x \in \Omega ;|x|<L+6, x_{3} \leq 0\right\} \\
& \Omega^{L}=\Omega_{+}^{L} \cup \Omega_{-}^{L} .
\end{aligned}
$$

Let

$$
u_{0}=R_{0}(z) \tilde{g} \text { if } \quad \operatorname{Im} z>0
$$

$$
\mathrm{u}_{0}=\mathrm{R}_{0}^{+}(\mathrm{z}) \tilde{\mathrm{g}} \quad \text { if } \quad z \in(0, \infty)
$$

Let $\mathbb{A}_{\mathrm{L}}$ be the restriction of $\mathcal{A}$ to $\Omega^{\mathrm{L}}$. v in $\mathrm{H}^{1}\left(\Omega^{\mathrm{L}}, \mathbb{C}^{3}\right)$ satisfies the generalized free boundary condition in $\Omega^{L}$ if and only if

$$
\int_{\Omega}{ }^{L}\left(e A_{L} v\right)_{i} \bar{w}_{i} \rho d x=\int_{\Omega}\left[\lambda(\nabla \cdot v)(\nabla \cdot \bar{w})+2 \mu \varepsilon_{i j}(v) \varepsilon_{i j}(\bar{w})\right] d x
$$

for every $w$ in $H^{1}\left(\Omega^{L}, \mathbb{C}^{3}\right)$.

The following operator $\left(A_{L}, D\left(A_{L}\right)\right)$ : 
$(5.41)$

$$
D\left(A_{L}\right)=\left\{v \in H^{1}\left(\Omega^{L}, \mathbb{C}^{3}\right) ; \mathbb{A}_{L} v \in L^{2}\left(\Omega^{L}, \mathbb{C}^{3}\right) \text { and } v \text { satisfies }(5.40)\right\}
$$

$$
A_{L} u=d A_{L} u, u \in D\left(A_{L}\right)
$$

is a positive selfadjoint one in $\mathrm{L}^{2}\left(\Omega^{\mathrm{L}}, \mathbb{c}^{3}, p d x\right)$.

Let $\psi_{1}$ and $\psi_{2}$ be two functions in $C_{0}^{\infty}\left(\mathbb{R}^{3}\right)$ such that

$(5.42) \quad \psi_{1}(x)=\left\{\begin{array}{lll}0 & \text { if } & |x|>L+2 \\ 1 & \text { if } & |x|<L+1\end{array}\right.$

(5.43) $\quad \psi_{2}(x)=\left\{\begin{array}{lll}0 & \text { if } & |x|>\text { L+5 } \\ 1 & \text { if } & |x|<L+4 .\end{array}\right.$

The lemma 5.1 shows that there exists $\tilde{u}_{0}$ in $H^{3}\left(\mathbb{R}^{3}, \mathbb{C}^{3}\right)$ such that $\psi_{2} \mathrm{u}_{0}-\tilde{\mathrm{u}}_{n}$ satisfies the generalized free boundary condition on $\partial \mathbb{R}_{+}^{3}$. $\tilde{u}_{0}$ can be choosen in such a way that its support is contained in $\left\{\mathrm{x} \in \mathbb{R}^{3} ; \mathrm{L}+3<|\mathrm{x}|<\mathrm{L}+6\right\}$.

Let $\Lambda$ be a complex number such that $\operatorname{Im} \Lambda<0$. Let

(5.44) $\left.\quad \mathrm{w}=6 \mathscr{A}_{0}-\Lambda\right)\left(\psi_{2} \mathrm{u}_{0}-\tilde{\mathrm{u}}_{0}\right)$ in $\Omega_{+}^{\mathrm{L}}$

(5.45) $\quad w=g$ in $\Omega_{-}^{\mathrm{L}}$.

Let

(5.46) $\quad \mathrm{w}^{\prime}=\left(\mathrm{A}_{\mathrm{L}}-\Lambda\right)^{-1} \mathrm{w}$.

We then have

$$
\left.\left(A_{L}-\Lambda\right) w^{\prime}=6 A_{0}-\Lambda\right)\left(\psi_{2} u_{0}-\tilde{u}_{0}\right) \text { in } \Omega_{+}^{L}
$$

$$
\left(A_{L}-\Lambda\right) w^{\prime}=g \text { in } \Omega_{-}^{L} \text {. }
$$


Seccond_-steeㄹ

Let

$$
h_{1}=\left.\mu_{0} \varepsilon_{13}\left(\left(1-\psi_{1}\right) u_{0}+\psi_{1} w^{\prime}\right)\right|_{x_{3}=0}
$$

$$
\begin{aligned}
& h_{2}=\left.\mu_{0} \varepsilon_{23}\left(\left(1-\psi_{1}\right) u_{0}+\psi_{1} w^{\prime}\right)\right|_{x_{3}=0} \\
& h_{3}=\left.\sigma_{33}\left(\left(1-\psi_{1}\right) u_{0}+\psi_{1} w^{\prime}\right)\right|_{x_{3}=0} .
\end{aligned}
$$

The support of $h={ }^{t}\left(h_{1}, h_{2}, h_{3}\right)$ is contained in $\left\{x \in \mathbb{R}^{3} ; L+1<|x|<L+2\right\}$. one easily verifies that $h$ is in $H^{3 / 2}\left(\mathbb{R}^{2}, \mathbb{C}^{3}\right)$. It follows from Lemma 5.1 that there exists $\tilde{u}=\mathscr{E}(\mathrm{h})$ such that

$$
\|\tilde{u}\|_{H^{3}\left(\mathbb{R}^{3}, \mathbb{C}^{3}\right)} \leq M\|g \mid\|_{0 ; s_{1}, s_{2}}
$$

for some positive constant $M$.

Furthermore $\tilde{\mathrm{u}}$ can be choosen in such way that its support is contained in $\left\{x \in \mathbb{R}^{3} ; L<|x|<L+3\right\}$.

\section{Thiiㅁ﹎. stẹep}

$$
\text { For any } z \text { in } \overline{\mathbb{C}}^{+} \text {let }
$$

$$
\mathrm{T}(\mathrm{z}) \mathrm{g}=\psi_{1}(\Lambda-z)\left(\mathrm{w}^{\prime}-\mathrm{u}_{0}\right)+\mathrm{D}\left(\psi_{1}, \mathrm{w}^{\prime}-\mathrm{u}_{0}\right)-\left(e \not_{0}-\mathrm{z}\right)^{\tilde{u}} \text { on } \Omega \cap \mathbb{R}_{+}^{3}
$$

$$
\mathrm{T}(\mathrm{z}) \mathrm{g}=(\Lambda-\mathrm{z}) \mathrm{w}^{\prime} \quad \text { on } \Omega \cap \mathbb{R}_{-}^{3}
$$

where $\quad \mathbb{R}_{-}^{3}=\left\{x \in \mathbb{R}^{3} ; \mathrm{x}_{3}<0\right\}$ and for $i=1,2,3$

(5.52) $\quad D_{i}(\psi, v)=-\sigma_{i j}(v) \frac{\partial \psi}{\partial x_{j}}-\frac{\partial}{\partial x_{j}}\left[\lambda_{0} \delta_{i j}\left(\sum_{k=1}^{3} v_{k} \frac{\partial \psi}{\partial x_{k}}\right)+\mu_{0}\left(v_{i} \frac{\partial \psi}{\partial x_{j}}+v_{j} \frac{\partial \psi}{\partial x_{i}}\right)\right]$

we have then the following lemma 
Lemma 5.3

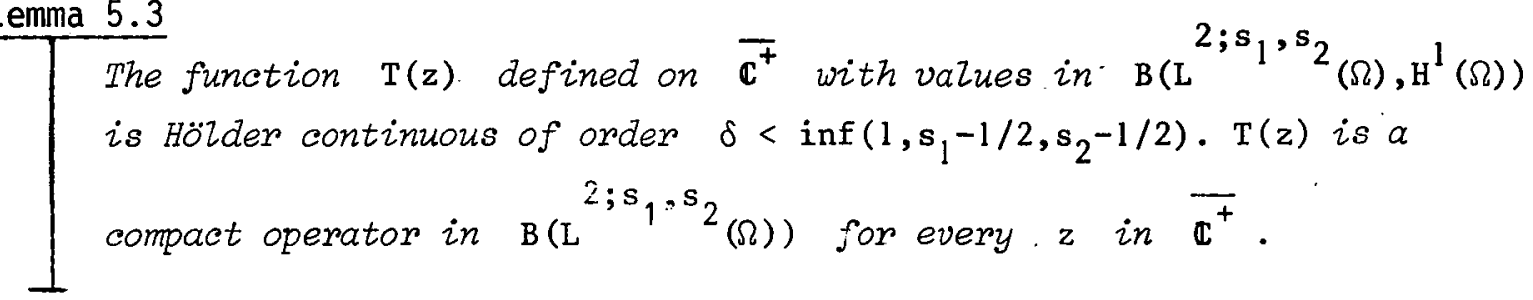

\section{Proof of Lemma 5.3}

Recall that $g$ is in $L^{2 ; s_{1}, s_{2}}(\Omega)$ and let $z$ and $z^{\prime}$ be in
$\mathbb{C}^{+}$. We then have

$$
|| w^{\prime}(z)-w^{\prime}\left(z^{\prime}\right)||_{H^{l}\left(\Omega^{L}\right)} \leq M|| R_{0}^{+}(z)-R_{0}^{+}\left(z^{\prime}\right)||_{B(L} 2 ; s_{1}, s_{2}\left(\mathbb{R}_{+}^{3}\right), H^{\left.2 ;-s_{1} 1^{-s_{2}}\left(\mathbb{R}_{+}^{3}\right)\right)}
$$

$$
. \mid\|g\|_{0 ; s_{1}, s_{2}}
$$

where $R_{0}^{+}(z)=R_{0}(z)$ if $\operatorname{Imz}>0$.

It then follows from theorem 3.7 that we have

$$
\left\|w^{\prime}(z)-w^{\prime}\left(z^{\prime}\right)\right\|_{H^{1}\left(\Omega^{L}\right)} \leq M\left|z-z^{\prime}\right|^{\delta}|| g \mid \|_{0 ; s_{1}, s_{2}}
$$

if $\delta<\inf \left(1, s_{1}-1 / 2, s_{2}-1 / 2\right)$.

From (5.54) we deduce that the first term of the r.h.s. of (5.51) has been estimated.

Let us remark that $\mathrm{w}^{\prime}$ belongs to $\mathrm{H}^{2}$ on $\{\mathrm{L}<|\mathrm{x}|<\mathrm{L}+3\}$. It then follows that we have

$$
\begin{aligned}
& \text { (5.55) } \quad|| w^{\prime}(z)-w^{\prime}\left(z^{\prime}\right)||_{H^{2}\left(\left\{L<|x|<L+3, c^{3}\right)\right.} \leq M\left|z-z^{\prime}\right|^{\delta}|||g|||_{0 ; s_{1}}, s_{2} \\
& \text { if } \delta<\inf \left(1, s_{1}-1 / 2, s_{2}-1 / 2\right) .
\end{aligned}
$$


Thus the second term of the r.h.s. of (5.51) is estimated. It is easy to estimate the third one because $\tilde{u}$ belongs to $H^{3}\left(\mathbb{R}^{3}\right)$. We get

$$
\left.|| \mathrm{T}(\mathrm{z}) \mathrm{g}\right|_{\mathrm{H}^{1}(\Omega)} \leq \mathrm{M}\||| \mathrm{g}\|_{0 ; s_{1}, s_{2}}
$$

and

$$
\left\|\mathrm{T}(\mathrm{z}) \mathrm{g}-\mathrm{T}\left(\mathrm{z}^{\prime}\right) \mathrm{g}\right\|_{\mathrm{H}^{1}(\Omega)} \leq \mathrm{M}\left|\mathrm{z}-\mathrm{z}^{\prime}\right|^{\delta}|||\mathrm{g}| \|_{0 ; \mathrm{s}_{1}, \mathrm{~s}_{2}} \text {. }
$$

Let $I^{+}(a, b)=\left\{z \in \overline{\mathbb{C}^{+}} ; a \leq \operatorname{Rez} \leq b\right\}$ and suppose that $[a, b] \subset(0, \infty)$ we finally get for $\delta<\inf \left(1, s_{1}-1 / 2, s_{2}-1 / 2\right)$ :

$$
|| T(z)-\left.T\left(z^{\prime}\right)\right|_{B\left(L^{2} ; s_{1}, s_{2}(\Omega), H^{1}(\Omega)\right)} \leq M_{\delta}\left|z-z^{\prime}\right|^{\delta}
$$

for every $\left(z, z^{\prime}\right)$ in $J^{+}(a, b) \times J^{+}(a, b)$.

The compactness of $T(z)$ considered as an operator in

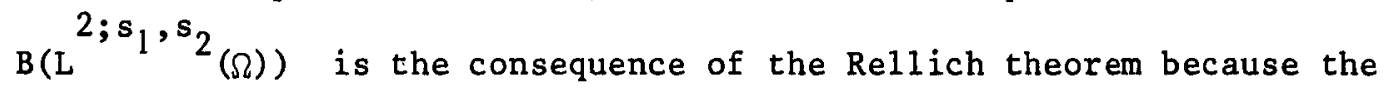
support of $T(z) g$ is contained in $\Omega^{\mathrm{L}}$ and $\Omega$ satisfies the cone condition.

\section{Fourth}

Let

$$
u=\left(1-\psi_{1}\right) u_{0}+\psi_{1} w^{\prime}-\tilde{u}
$$

One easily checks that $u$ is in $\mathrm{H}^{1 ;-\mathrm{s}_{1},-\mathrm{s}_{2}}\left(\Omega, A, \mathbb{C}^{3}\right)$ and that

$$
\text { (5.60) } \quad(d-z) u=g+T(z) g .
$$

Let

$$
\overline{\mathbf{C}_{c}^{+}}=\left\{z \in \overline{\mathbb{C}^{+}} ; z \text { is not a strictly positive eigenvalue of } A\right\} \text {. }
$$


We then have :

Lemma 5.4

$I$ For any $\mathrm{z}$ in $\overline{\mathrm{c}_{\mathrm{c}}^{+}}, \mathrm{I}+\mathrm{T}(\mathrm{z})$ is invertible in $\mathrm{B}\left(\mathrm{L}^{2 ; \mathbf{s}_{1}, \mathrm{~s}_{2}}(\Omega)\right)$.

The proof of lemma 5.4 is by contradiction.

Suppose there exists $g$ in $\mathrm{L}^{2 ; \mathrm{s}_{1}, s_{2}}(\Omega), \mathrm{g} \neq 0$ and $\mathrm{z}$ in $\overline{\mathbb{C}_{\mathrm{c}}^{+}}$ such that

(5.61) $g+T(z) g=0$.

From (5.60) we deduce that

(5.62) $\quad(e A-z) u=0$

where $u$ is in $H^{1 ;-s_{1},-s_{2}}\left(\Omega, A, \mathbb{C}^{3}\right)$.

If we prove that $u$ is different from zero and is an eigenfunction of $A$ associated with the eigenvalue $z$, this will be a contradiction because we have supposed that $z$ belongs to $\overline{\mathbb{C}_{c}^{+}}$.

Now suppose $g \neq 0$ and $u=0$. We then have $w^{\prime}=0$ in $\left\{x \in \Omega^{L} ;|x|<L\right\}$. From (5.48) it follows that $g=0$ in $\Omega_{-}^{\mathrm{L}}$. In particular $w^{\prime \prime}$ can be extended to $B_{+}=\left\{x \in \mathbb{R}_{+}^{3} ;|x|<L+6\right\}$ by letting

$$
\tilde{w}^{\prime}=w^{\prime} \text { in } \Omega^{L}
$$

$$
\tilde{\mathrm{w}}^{\prime}=0 \text { in } \mathrm{B}_{+} \backslash \Omega^{\mathrm{L}} \text {. }
$$

One easily verifies that $\tilde{w}^{\prime}$ is in $H^{2}\left(B_{+}\right)$and satisfies the free boundary condition on $\partial \mathrm{B}_{+}$. 
We have in $\Omega_{+}^{L}$

$$
\left(A_{0}-\Lambda\right) w^{\prime}=\left(A_{L}-\Lambda\right) w^{\prime}=\left(A A_{0}-\Lambda\right)\left(\psi_{2} u_{0}-\tilde{u}_{0}\right)
$$

\section{Therefore}

$$
\begin{aligned}
& \left(A_{0}-\Lambda\right)\left(\tilde{w}^{\prime}-\psi_{2} u_{0}+\tilde{u}_{0}\right)=0 \text { in } \Omega_{+}^{L} \\
& \left(A_{0}-\Lambda\right)\left(\tilde{w}^{\prime}-\psi_{2} u_{0}+\tilde{u}_{0}\right)=-\left(e A_{0}-\Lambda\right) u_{0} \text { in } B_{+} \backslash \Omega_{+}^{L} .
\end{aligned}
$$

Let

$$
\omega=\tilde{w}^{\prime}-\psi_{2} u_{0}+\tilde{u}_{0}
$$

Let $\tilde{\mathrm{A}}_{0}$ be the positive selfadjoint operator in $L^{2}\left(B_{+}, \mathbb{C}^{3}, \rho_{0} \mathrm{dx}\right)$ associated with $A_{0}$ and the free boundary condition on $\partial \mathrm{B}_{+} \cdot \omega$ is then in the domain of $\tilde{\mathrm{A}}_{0}$. It follows from (5.65) that $\tilde{\mathrm{A}}_{0} \omega=\Lambda \omega$ in $\Omega_{+}^{L}$ and from (5.66) that $\omega=-\mathrm{u}_{0}$ in $\mathrm{B}_{+} \backslash \Omega_{+}^{\mathrm{L}}$. Recall that $\mathcal{A}_{0} \mathrm{u}_{0}=\mathrm{zu}_{0}$ in $B_{+} \backslash \Omega_{+}^{L}$. We then have

$$
\int_{B_{+}}\left(\lambda_{0}|\nabla \cdot \omega|^{2}+2 \mu_{0} \sum_{i, j=1}^{3}\left|\varepsilon_{i j}(\omega)\right|^{2}\right) d x=\int_{B_{+}} \widetilde{A}_{0} \omega \cdot \bar{\omega} \rho_{0} d x
$$

$$
\begin{aligned}
& =\int_{\Omega_{+}^{L}} \tilde{A}_{0} \omega \cdot \bar{\omega} \rho_{0} \mathrm{dx}+\int_{\mathrm{B}_{+} \backslash \Omega_{+}^{L}} \widetilde{\mathrm{A}}_{0} \omega \cdot \bar{\omega} \rho_{0} \mathrm{dx} . \\
& =\Lambda \int_{\Omega_{+}^{L}}|\omega|^{2} \rho_{0} \mathrm{dx}-z \int_{\mathrm{B}_{+} \backslash \Omega_{+}^{L}}\left|\mathrm{u}_{0}\right|^{2} \rho_{0} \mathrm{dx} .
\end{aligned}
$$

Thus the r.h.s. of (5.67) is a real number and $\omega=0$ in $\Omega_{+}^{L}$ because we know that $\operatorname{Im} \Lambda$ is strictly negative and $\operatorname{Imz}$ is non negative.

Therefore 
$(5.68)$

$$
\tilde{w}^{\prime}=\psi_{2} u_{0}-\tilde{u}_{0} \text { in } \Omega_{+}^{L}
$$

$$
u=\left(1-\psi_{1}\right) u_{0}+\psi_{1} \psi_{2} u_{0}-\psi_{1} \tilde{u}_{0}-\tilde{u} \text { in } \Omega_{+}^{L}
$$

Remark that

$$
\psi_{1} \psi_{2}=\psi_{1} \text { and } \psi_{1} \tilde{u}_{0}=0
$$

Therefore

$$
u=u_{0}-\tilde{u} \text { in } \Omega_{+}^{L}
$$

(5.71) implies that $\tilde{u}$ satisfies the free boundary condition on $\partial \mathbb{R}_{+}^{3}$. Thus $h$ defined by $(5.49)$ is equal to zero and $\tilde{u}=0$. Finally $u=u_{0}=0$ in $\Omega_{+}^{L^{2}}$. We then deduce that $\tilde{u}_{0}=0$ and $\tilde{w}^{\prime}=0$ in $\Omega_{+}^{\mathrm{L}}$. From (5.51) and (5.61) we conclude that $g=0$. Then, if $\operatorname{Imz}>0,(5.59)$ and (5.62) show that $u$ belongs to $D(A)$ and is an eigenfunction of $A$ associated with the eigenvalue $z$. This is impossible because $A$ is a selfadjoint operator.

Now suppose $z=\sigma^{2}>0$ and $\sigma^{2} \in \overline{\mathbb{d}_{c}^{+}}$. In this case $u$ is an eigenfunction of $A$ associated with the eigenvalue $\sigma^{2}$. Indeed $I=\left\langle\tilde{g}, R_{0}^{+}\left(\sigma^{2}\right) \tilde{g}\right\rangle$ is a real number. This is proved exactly in the same way as in the last part of the first proof of theorem 5.2. We then have

$$
\tau_{P}(\sigma) \tilde{g}=\tau_{S V}(\sigma) \tilde{g}=\tau_{S V}^{0}(\sigma) \tilde{g}=\tau_{S H}(\sigma) \tilde{g}=\tau_{R}(\sigma) \tilde{g}=0
$$

It follows from (5.72) and theorem 4.1 that $u_{0}$ is in $L^{2}\left(\mathbb{R}_{+}^{3}, \mathbb{C}^{3}, \rho_{0} \mathrm{dx}\right)$. Therefore $u$ is $\frac{\text { in }}{+} \mathrm{D}(\mathrm{A})$. But this is impossible because we have supposed that $\sigma^{2}$ is in $\overline{C_{c}^{+}}$. In conclusion we have $g=0$ and the lemma 5.4 is proved by using the compactness of $T(z)$ Q.E.D.
a 
It is now easy to conclude the second proof of theorem 5.2.

Given $f$ in $L^{2 ; s_{1}, s_{2}}(\Omega)$ and $z$ in ${\overline{C_{C}^{+}}}^{+}$we first solve the following equation

$$
\mathrm{f}=(1+\mathrm{T}(\mathrm{z})) \mathrm{g} \text {. }
$$

It results from lemma 5.4 that there exists an unique $g$ in $L^{2 ; s_{1}, s_{2}}(\Omega)$ solution of (5.73).

From $g$ and the first two steps and from (5.59) we construct $u$. If $\operatorname{Imz}>0, u_{0}$ is in $H^{2}\left(\mathbb{R}_{+}^{3}, \mathbb{C}^{3}\right)$ and $u$ is in $D(A)$. From (5.60) we then have

(5.74) $u=R(z) f$.

If $\operatorname{Im} z=0$ we define $\mathrm{R}^{+}(z)$ by

(5.75) $\quad u=R^{+}(z) f$.

Finally by using (5.73), (5.74), (5.75) and lemma 5.3 we easily prove for $\delta<\inf \left(1, s_{1}-1 / 2, s_{2}-1 / 2\right)$ that

$$
\left\|\mathrm{R}^{+}(\mathrm{z})-\mathrm{R}^{+}\left(\mathrm{z}^{\prime}\right)\right\|_{\mathrm{B}(\mathrm{L}} 2 ; \mathrm{s}_{1}, \mathrm{~s}_{2}(\Omega), \mathrm{L}^{\left.2 ;-\mathrm{s}_{1},-\mathrm{s}_{2}(\Omega)\right)} \leq \mathrm{M}_{\delta}\left|z^{-z^{\prime}}\right|^{\delta}
$$

for some positive constant $M$ depending of $\delta$ and for $\left(z, z^{\prime}\right) \in J^{+}(a, b) x$ $\mathrm{J}^{+}(\mathrm{a}, \mathrm{b})$ where $[\mathrm{a}, \mathrm{b}]$ is contained in $\overline{\mathrm{C}_{\mathrm{C}}^{+}}$. In $(5.76)$ we have let $\mathrm{R}^{+}(z)=\mathrm{R}(\mathrm{z})$ if $\operatorname{Im} z>0$.

of course by considering $\overline{C_{c}}=\{z \in \mathbb{C} ; \operatorname{Imz} \leq 0, z \neq 0$ and $z$ is not an eigenvalue of $A\}$ we get a similar result for $R^{-}(z)$.

This concludes the second proof of theorem 5.2.

Q.E.D. 
Finally by using theorem 4.1 and theorem 5.2 and as in [6] we prove the following theorem.

Theorem 5.5

(i) A has no continuous singular spectmu.

(ii) If $[\mathrm{a}, \mathrm{b}]$ is a compact interval contained in $(0, \infty)$

A can only have a finite number of eigenvalues in [a,b] and each of these eigenvalues has a finite multiplicity.

(iii) Let $\mathbf{u}$ be any eigenfunction of $\mathrm{A}$ associated with a strictly positive eigenvalue. We then have

(5.77) $u \in \underset{s<2}{n} L^{2}\left(\Omega, c^{3},\left(1+x_{3}\right)^{s} d x\right)$.

In conclusion let us note that for perturbations of $A_{0}$ in $\mathbb{R}_{+}^{2}$ the results given in theorems 5.2 and 5.5 are the same as for perturbations of $A_{0}$ in $\mathbb{R}_{+}^{3}$. 
[1] J.D. ACHENBACH, Wave propagation in elastic solids. North Holland, Amsterdam, 1973.

[2] J.D. ACHENBACH, A.K. GANTESEN and H. MCMAKEN. Ray methods for waves in elastic solids with applications to scattering by cracks. Pitman, 1982.

[3] S. AGMON. Spectral properties of Schrödinger operators and scattering theory. Ann. Scuola Norm. Sup. Pisa Ser. IV, $\underline{2}$ (1975), 151-218.

[4] K. AKI and P.G. RICHARDS. Quantitative seismology. Theory and methods. Vol. I and Vol. II. Freeman, 1980.

[5] B.A. AULD. Acoustic fields and waves in solids. Vol. I and Vol. II. Wiley Interscience, 1973.

[6] Y. DERMENJIAN and J.C. GUILLOT. Théorie spectrale de la propagation des ondes acoustiques dans un milieu stratifié perturbé. 1984, to be published in the "Journal of Differential Equations".

[7] Y. DERMENJIAN and J.C. GUILLOT. Les ondes élastiques dans un demiespace isotrope. Développement en fonctions propres généralisées. Principe d'absorption limite. C.R.A.S., 300 (1985), 93-96.

[8] Y. DERMENJIAN and J.C. GUILlOT. Théorie de la diffusion des ondes élastiques dans un demi-espace isotrope perturbé. C.R.A.S., 301 (1985), 617-619.

[9] N. DUNFORD and J.T. SCHWARTZ. Linear operators. Part II, Spectral theory. Interscience Publishers, New York, 1963. 
[10] G. DUVAUT and J.L. LIONS. Les inéquations en mécanique et en physique. Dunod, Paris, 1972.

[11] A.G. ERINGEN and E.S. SUHUBI. Elastodynamics. Vol. II Linear theory. Academic Press, 1975.

[12] D.M. EIDUS. The principle of limiting absorption, A.M.S., Transl. (2) 47 (1965), 157-191.

[13] D.M. EIDUS. The principle of limiting amplitude, Russian Math. Surveys 24 (1969), 97-167.

[14] J. GOBERT. Une inéquation fondamentale de la théorie de l'élasticité, Bu11. Soc. Roy. Sci. Liège, 3lè année, n 3-4 (1962), 182-191.

[15] J.C. GUILLOT and C.H. WILCOX. Spectral analysis of the Epstein operator, Proc. Roy. Soc. Edinburgh 80A(1978), 85-98.

[16] J.C. GUILLOT. Théorie spectrale et développement en fonctions propres pour les équations de Maxwell dans une couche diélectrique Rapport $\mathrm{n}^{\circ}$ 385, I.N.R.I.A., 78153 Le Chesnay, France, 1985.

[17] J.C. GUILLOT. Existence and uniqueness of a Rayleigh surface wave propagating along the free boundary of a transversely isotropic elastic half space. To be published in Math. Math. Appl. Sci.

[18] L. HORMANDER. The analysis of linear partial differential operators II. Springer, Berlin, 1983.

[19] T. KATO. Perturbation theory for linear operators. Springer, New York, 1966. 
[20] A. MAJDA. Outgoing solutions for perturbations of $-\Delta$ with applications to spectral and scattering theory. J. Diff. Eq. 16 (1974) 515-547.

[21] S. MIZOHATA. The theory of partial differential equations. Cambridge University Press, Londres, 1973.

[22] R. PHILLIPS. On the exterior problem for the reduced wave equation. A.M.S. Summer Institute in Partial Differential Equations, Berkeley, 1971 .

[2ミ] M. REED and B. SIMON. Methods of Modern Mathematical Physics. Vol. II Fourier Analysis, Self Adjointness. Academic Press, New York, 1975.

[24] M. REED and B. SIMON. Methods of Modern Mathematical Physics. Vo1. III. Scattering Theory. Academic Press, New York, 1978.

[25] M. REED and B. SIMON. Methods of Modern Mathematical Physics. Vo1. IV. Analysis of Operators. Academic Press, New York, 1978.

[26] M. ROSEAU. Asymptotic wave theory. North Holland, 1976.

[27] M. SCHECHTER. Operator Methods in Quantum Mechanics. E1sevier North Holland, 1981 .

[28] J.R. SCHULENBERGER. Elastic waves in the half space $\mathbb{R}_{+}^{2}$. J. Diff. Eq. $\underline{29}$ (1978), 405-438.

[29] R. WEDER. Spectral and scattering theory in perturbed stratified fluids, II, Transmission problems and exterior domains. Communicaciones Técnicas IIMAS-UNAM. Serie Naranja n $368,1984$. 
[30] C.H. WILCOX. Scattering theory for the d'Alembert equation in exterior domains. Lecture Notes in Mathematics, $\mathfrak{n}^{\circ} 442$, Springer Berlin, 1975 .

[31] C.H. WILCOX. Spectral analysisiof the Pekeris operator. Arch. Rational Mech. Anal. 60 (1976), 259-300.

[32] C.H. WILCOX. Sound propagation in stratified media. Applied Mathematical Sciences, Vol. 50, Springer, 1984.

[33] C.H. WILCOX. Transient electromagnetic wave propagation in a dielectric waveguide. Istituto Nazionale di Alta Matematica, Symposia Matematica XVIII, 1976, 239-277. 
